Cavity Resonator for Permittivity Measurements of Liquids at Millimeter-wave Frequencies

A Dissertation
Presented to
the faculty of the School of Engineering and Applied Science
University of Virginia
in partial fulfillment
of the requirements for the degree
Doctor of Philosophy
by

Angelique Helene Sklavounos

December

2013 
APPROVAL SHEET

The dissertation

is submitted in partial fulfillment of the requirements

for the degree of

Doctor of Philosophy

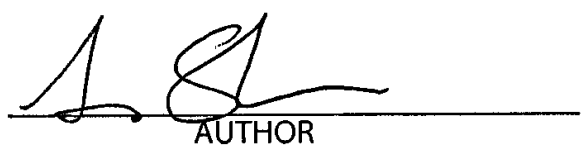

The dissertation has been read and approved by the examining committee:

N. Scott Barker

Advisor

Robert Weikle III

Brooks Pate

Tatiana Globus

Avik Ghosh

Accepted for the School of Engineering and Applied Science:

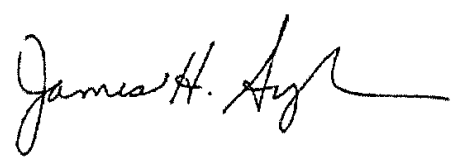

Dean, School of Engineering and Applied Science

December

2013 


\section{Abstract}

Permittivity data of liquids is necessary for applications such as dielectric heating, remote sensing, and moisture detection, and is also used for molecular characterization. Dispersive molecular mechanisms occur for field excitations of frequencies mainly above $10 \mathrm{GHz}$ and extending into terahertz and optical frequencies. Around $100 \mathrm{GHz}$ there is less data, due to the frequency limits of microwave and quasi-optical techniques. This work presents an over-moded cavity resonator for liquid permittivity measurements. Novel full-wave modeling of a four-port inhomogeneous waveguide junction removes the limits imposed by previous methods. A cavity with environmental control was designed and tested. The parameters estimated from the modeling and measurement inputs are plausible and comparable to literature. Based on repeatability measurements and a sensitivity analysis, recommendations are made for future cavity designs that will enable permittivity measurements at frequencies previously little measured. 


\section{Acknowledgements}

I would foremost like to thank Dr. Scott Barker for his guidance, encouragement, and patience, particularly while I wrestled with the modeling. His observations and advice always helped to keep me afloat. I would also like to thank Dr. Robert Weikle III for providing helpful consultations with the modeling, along with Dr. Brooks Pate, Dr. Tatiana Globus, and Dr. Avik Ghosh for serving on my committee and for their helpful knowledge with regards to spectroscopy and liquid measurement.

I also want to thank all of the former and present members of MiRFTech and the FIR lab for all of their thoughtful instruction and advice, as well as crucial camaraderie. In particular, Dr. Lihan Chen was always willing to answer my random questions, Matthew Bauwens was of great help with the block design, and Dr. Alex Arsenovic was influential with the Python programming. Thanks must also go the staff of the ECE department for their aid and friendliness.

Much gratitude to my friends Alex, Robert, Nii, and Vishal for fun trips and non sequitur e-mails, all of which never failed to amuse and recharge me. And last but not least, the deepest appreciation to my parents for their eternal love and support. I dedicate this work to them. 


\section{Contents}

List of Figures viii

List of Tables $\quad$ ix

List of Symbols $\quad x$

$\begin{array}{lll}1 & \text { Background } & 1\end{array}$

1.1 Physical Origin of Permittivity $\ldots \ldots \ldots \ldots \ldots \ldots \ldots$

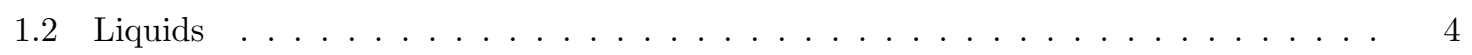

1.2 .1 Water . . . . . . . . . . . . . . . . . . . 5

1.3 Measurement techniques $\ldots \ldots \ldots \ldots \ldots \ldots \ldots$

1.3 .1 Terahertz . . . . . . . . . . . . . . . . . . . . 6

1.3 .2 Microwave . . . . . . . . . . . . . . . . . . 8

1.4 Conclusion and Thesis Overview $\ldots \ldots \ldots \ldots \ldots \ldots$

2 Cavity Design and Modeling 11

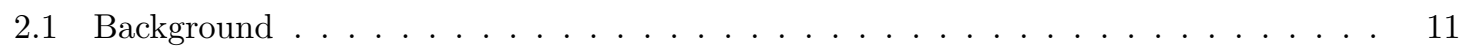

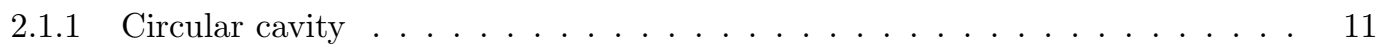

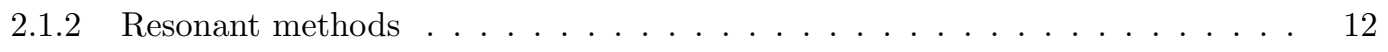

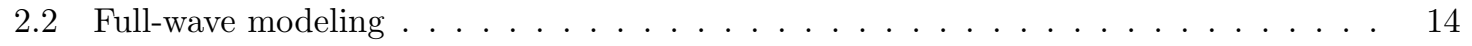

2.2 .1 Mode-matching . . . . . . . . . . . . . . . . . . . . . . . . 15

2.2 .2 Modal indices . . . . . . . . . . . . . . . . . . . . . . . . . . . . . . . 18

$2.2 .3 \quad$ Generalized Scattering Matrix $\ldots \ldots \ldots \ldots \ldots$ 
2.3 Modeling of four port aperture-coupled cavity junction $\ldots \ldots \ldots \ldots$. . . . . . . 20

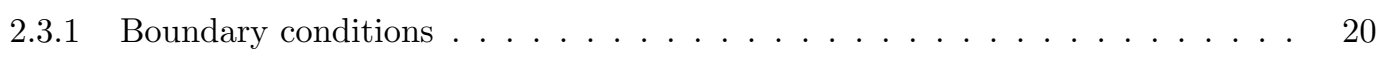

2.3 .2 Inhomogeneous propagation constants $\ldots \ldots \ldots \ldots \ldots$

2.3 .3 Bessel conditioning . . . . . . . . . . . . . . . . . . . . . . 24

$2.3 .4 \quad$ Aperture approximation $\ldots \ldots \ldots \ldots \ldots \ldots \ldots \ldots$

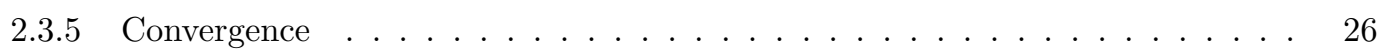

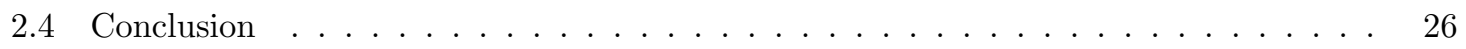

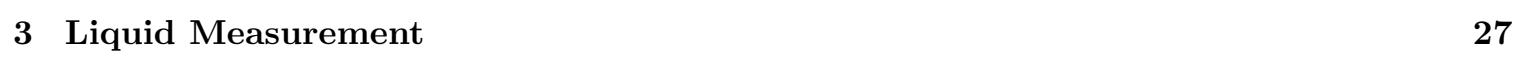

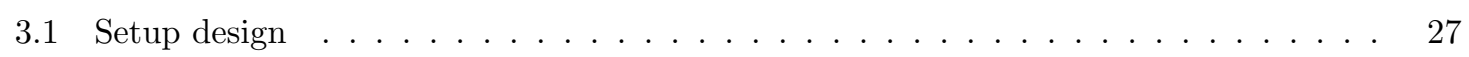

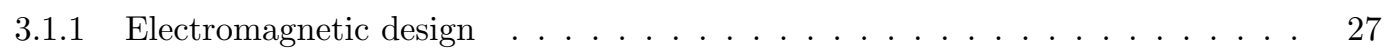

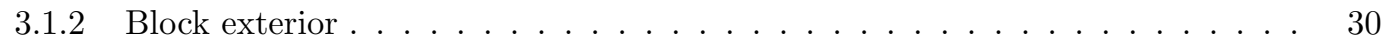

3.2 Measurement . . . . . . . . . . . . . . . . . . . . . . . . . . . . . . . . 33

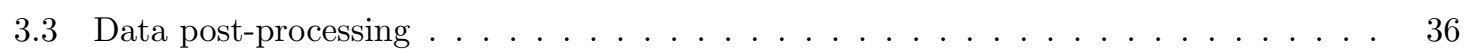

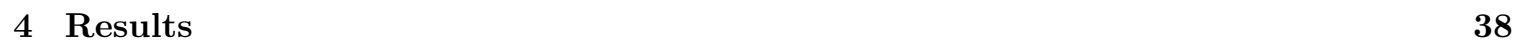

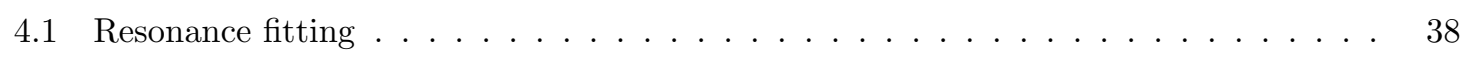

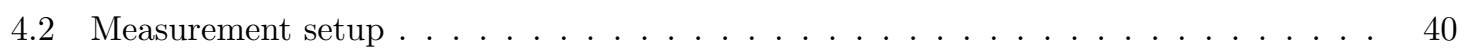

4.2 .1 Environmental controls $\ldots \ldots \ldots \ldots \ldots \ldots$. . . . . . . . . . . . 40

$4.2 .2 \quad$ Data variability $\ldots \ldots \ldots \ldots \ldots \ldots \ldots$

4.2 .3 Repeatability tests $\ldots \ldots \ldots \ldots \ldots \ldots \ldots \ldots$. . . . . . . . . . . 45

4.3 Modeling . . . . . . . . . . . . . . . . . . . . . . . . . . . . . . 48

4.3 .1 Validation $\ldots \ldots \ldots \ldots \ldots \ldots \ldots$

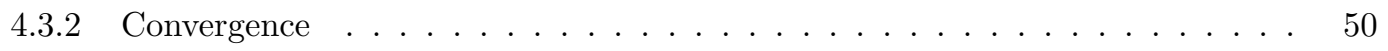

$4.3 .3 \quad$ Aperture approximation $\ldots \ldots \ldots \ldots \ldots \ldots \ldots \ldots$

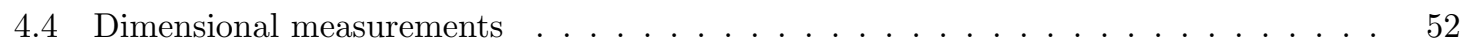

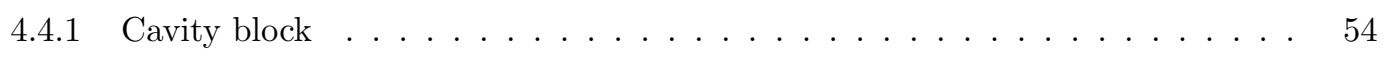

4.4 .2 Tube $\ldots \ldots \ldots \ldots \ldots$

4.5 Liquids $\ldots \ldots \ldots \ldots \ldots$

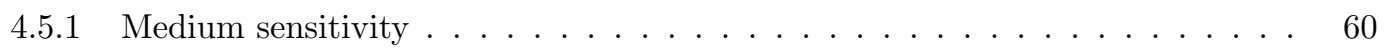


4.5 .2 Low sensitivity $\ldots \ldots \ldots \ldots \ldots \ldots \ldots \ldots \ldots$

4.5 .3 Mode-mixing . . . . . . . . . . . . . . . . . . . . 65

4.6 Permittivity Data . . . . . . . . . . . . . . . . . . . . . . . . . 66

4.6 .1 Uncertainty . . . . . . . . . . . . . . . . . . . . . . . . . . . . . . . . . 69

4.6 .2 Repeatability . . . . . . . . . . . . . . . . . . . 70

$\begin{array}{lll}5 & \text { Conclusions and Future Work } & 72\end{array}$

\begin{tabular}{ll}
\hline A Equations & 75
\end{tabular}

A.1 Waveguide . . . . . . . . . . . . . . . . . . . . . . . . . . . . 75

A.2 Fields in Cavity and Hole regions . . . . . . . . . . . . . . . . . . 78

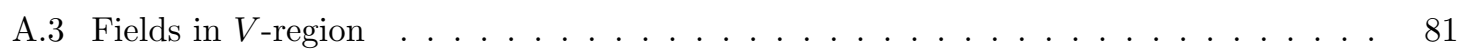

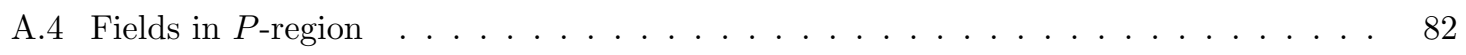

A.5 Fields in rectangular waveguide regions $\ldots \ldots \ldots \ldots$

A.5.1 Rectangular-to-cylindrical coordinate transformation . . . . . . . . . . . 86

A.6 Full boundary conditions . . . . . . . . . . . . . . . . . . . . . . . . . . . . 88

A.6.1 Analytical Integrations . . . . . . . . . . . . . . . . . . . . . . . 89

A.6.2 Small-angle approximation . . . . . . . . . . . . . . . . 90

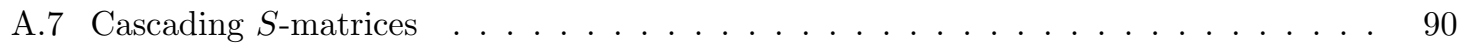

A.8 Symmetrical $S$-matrix $\ldots \ldots \ldots \ldots \ldots$

\begin{tabular}{ll}
\hline B Drawings & 94
\end{tabular}

\begin{tabular}{ll}
\hline C Microwave PCR & 104 \\
\hline
\end{tabular}

C.1 Microchip design $\ldots \ldots \ldots \ldots \ldots \ldots$

C.2 Fluidic microchip $\ldots \ldots \ldots \ldots \ldots \ldots \ldots \ldots$

C.3 Electrical design . . . . . . . . . . . . . . . . . . . . . . . . . . . . . 109

\begin{tabular}{ll}
\hline Bibliography & 114
\end{tabular} 


\section{List of Figures}

1.1 Diagram: orientational polarization . . . . . . . . . . . . . . . . . . . . . 3

1.2 Diagram: vibrational modes . . . . . . . . . . . . . . . . . . . . . . . . . . . . 3

1.3 Plot: dispersion . . . . . . . . . . . . . . . . . . . . . . . . . . . . . 4

$2.1 \quad$ Diagram: circular cavity geometry . . . . . . . . . . . . . . . . . . . . . 13

2.2 Diagram: cross-section of cavity block and tube . . . . . . . . . . . . . . . . . . . . . 15

2.3 Diagram: mode-matching waveguide discontinuity . . . . . . . . . . . . . . . . . . . 16

2.4 Diagram: modeling discontinuities . . . . . . . . . . . . . . . . . . . . . . . . . . . 21

2.5 Diagram: discontinuities to $S$-matrices . . . . . . . . . . . . . . . . . . . . . . . . . . 23

2.6 Diagram: complex root search . . . . . . . . . . . . . . . . . . . . . . . . . 25

$3.1 \quad$ Diagram: cavity block negative space . . . . . . . . . . . . . . . . . . . . . . . . . . . 29

3.2 Plot: TE mode electric field plots . . . . . . . . . . . . . . . . . . . . . . . . . . 30

3.3 CAD: block exterior 1 . . . . . . . . . . . . . . . . . . . . . . . . . . . . . . . . . . . 31

3.4 CAD: block exterior 2 . . . . . . . . . . . . . . . . . . . . . . . . . . . . . . 31

3.5 Photo: Quartz tube fluidics . . . . . . . . . . . . . . . . . . . . . . . . 31

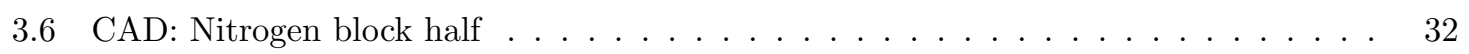

3.7 Photo: Nitrogen block . . . . . . . . . . . . . . . . . . . . . . . . . . . . . . . . . . 32

3.8 Plot: Nitrogen inlet hole return loss . . . . . . . . . . . . . . . . . . . . . . . . . . 33

3.9 Photo: Measurement setup . . . . . . . . . . . . . . . . . . . . . . . . 35

3.10 Photo: Inner insulation . . . . . . . . . . . . . . . . . . . . . . . . 35

3.11 Photo: Outer insulation . . . . . . . . . . . . . . . . . . . . . . . . 35 
3.12 Photo: Liquid measurement . . . . . . . . . . . . . . . . . . . . . . . 36

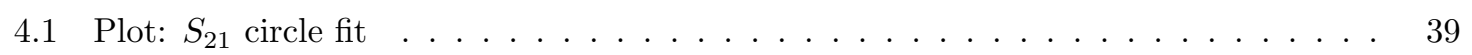

4.2 Plot: Phase fit . . . . . . . . . . . . . . . . . . . . . . . . . . . . . . . . . . 39

$4.3 \quad$ Plot: $S$-parameters of $\mathrm{N}_{2}$ blocks $\ldots \ldots \ldots \ldots \ldots \ldots \ldots \ldots \ldots$

4.4 Plot: Calibrated $S$-parameters $\ldots \ldots \ldots \ldots \ldots \ldots$. . . . . . . . . . . . . 41

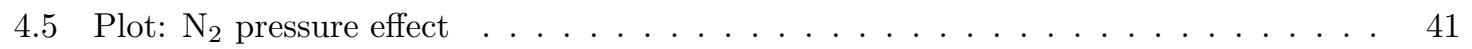

4.6 Plot: Temperature control $\ldots \ldots \ldots \ldots \ldots \ldots$. . . . . . . . . . . . . . 42

4.7 Plot: Temperature stability and multiple setpoints . . . . . . . . . . . . . . 42

4.8 Plot: Multi-day data of $\mathrm{TE}_{811}$ mode at $30^{\circ} \mathrm{C}, \mathrm{Al}$ block $\ldots \ldots \ldots \ldots$. . . . . . 44

4.9 Plot: Multi-day data of $\mathrm{TE}_{811}$ mode at $28^{\circ} \mathrm{C}, \mathrm{Au}$ block $\ldots \ldots \ldots \ldots \ldots$

4.10 Plot: Measurement of cavity left on VNA $\ldots \ldots \ldots \ldots$

4.11 Plot: Resonance sweeps, cavity reassembly . . . . . . . . . . . . . . . . . . 47

4.12 Plot: Resonance data, cavity reattachment . . . . . . . . . . . . . . . . 47

4.13 Plot: W-band $S_{21}$ measurement v. modeling $\ldots \ldots \ldots \ldots \ldots$. . . . . . 48

4.14 Plot: W-band phase measurement vs. modeling . . . . . . . . . . . . . . . . 49

4.15 Plot: $\mathrm{TE}_{811}$ measured vs. modeling. $\ldots \ldots \ldots \ldots$. . . . . . . . . . 49

4.16 Diagram: modal limits by dimensional ratios $\ldots \ldots \ldots \ldots \ldots \ldots$

4.17 Plot: $\mathrm{TE}_{221}$ convergence $\ldots \ldots \ldots \ldots \ldots \ldots \ldots \ldots$. . . . . . . . . . . . . . . . .

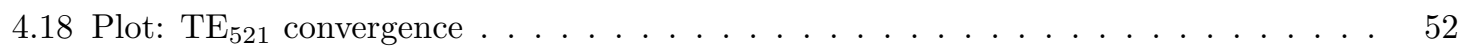

4.19 Plot: Small-angle approximation vs. numerical integrals, $\mathrm{TE}_{221} \ldots \ldots \ldots \ldots$

4.20 Plot: Small-angle approximation vs. numerical integrals, $\mathrm{TE}_{521} \ldots \ldots \ldots$. . . . . . . 53

4.21 Diagram: Measured block dimensions $\ldots \ldots \ldots \ldots \ldots$

4.22 Plot: Simulated conditions of three resonances $\ldots \ldots \ldots \ldots$

4.23 Plot: $f_{0}$ sensitivity to aperture angle offset $\ldots \ldots \ldots \ldots \ldots \ldots$

4.24 Plot: Tube inner radius determination $\ldots \ldots \ldots \ldots \ldots \ldots$

4.25 Photo: Microscope image of tube $\ldots \ldots \ldots \ldots$

4.26 Plot: Literature fused quartz permittivity $\ldots \ldots \ldots \ldots \ldots$

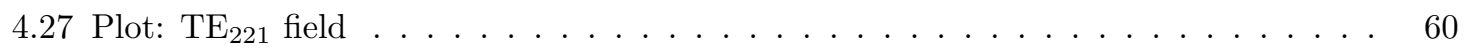

4.28 Plot: Methanol I measurements \& simulation . . . . . . . . . . . . . . . . . . . . 60 
4.29 Plot: Water $\mathrm{TE}_{221}$ measurements \& simulation . . . . . . . . . . . . . . . . . . . . . 62

4.30 Plot: Literature permittivity data of water . . . . . . . . . . . . . . . . . . . . . . 62

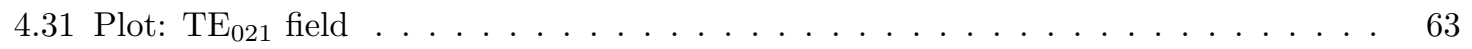

4.32 Plot: Methanol $\mathrm{TE}_{021}$ measurements \& simulation . . . . . . . . . . . . . . . . . . 63

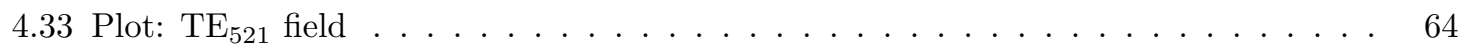

4.34 Plot: Water $\mathrm{TE}_{521}$ measurements \& simulation . . . . . . . . . . . . . . . . . . . . 64

4.35 Plot: $\mathrm{TE}_{711}$ measurements \& simulation . . . . . . . . . . . . . . . . . . . . . . . . . 65

4.36 Plot: Simulated cavity and tube of $\mathrm{TE}_{131}$ and $\mathrm{TE}_{711}$. . . . . . . . . . . . . . . . . 66

4.37 Plot: $\mathrm{TE}_{711}$ and $\mathrm{TE}_{131}$ fields . . . . . . . . . . . . . . . . . . . . . . 67

4.38 Plot: Methanol permittivity data . . . . . . . . . . . . . . . . . . . 68

5.1 Plot: TM vs. TE field plots . . . . . . . . . . . . . . . . . . . . . . . . . 73

5.2 Diagram: TM block spit . . . . . . . . . . . . . . . . . . . . . . . . . . . 74

A.1 Rectangular waveguide. . . . . . . . . . . . . . . . . . . . . . . . . . 78

A.2 Circular waveguide. . . . . . . . . . . . . . . . . . . . . . . . . . . . . . 78

A.3 Diagram: modeling discontinuities . . . . . . . . . . . . . . . . . . . . . . . . . . 79

A.4 Diagram: coordinate systems in cavity and aperture . . . . . . . . . . . . . . . . . . 87

A.5 Combination of two cascaded S-matrices. . . . . . . . . . . . . . . . . . . . . . . . 90

A.6 Diagram: Symmetrical 2-port from 1-port . . . . . . . . . . . . . . . . . . . . . . . 93

A.7 Diagram: Symmetrical 4-port from 2-port . . . . . . . . . . . . . . . . . . . . . 93

C.1 Drawing: PCR microwave microchip . . . . . . . . . . . . . . . . . . . . . . . . . . . 106

C.2 Diagram: PCR microwave microchip . . . . . . . . . . . . . . . . . . . . . . . . . . . 107

C.3 Plot: PCR chamber temperature distributions . . . . . . . . . . . . . . . . . . . . . . 109

C.4 Photo: Experimental PCR microchip on ground plane . . . . . . . . . . . . . . . . . 111

C.5 Plot: $S_{11}$ measurement of matched PCR chamber. . . . . . . . . . . . . . . . . . . . 112

C.6 Diagram: HFSS model of PCR chamber . . . . . . . . . . . . . . . . . . . . . . . . . 112

C.7 Plot: Smith Chart of HFSS and RC load impedances . . . . . . . . . . . . . . . . . . 113 


\section{List of Tables}

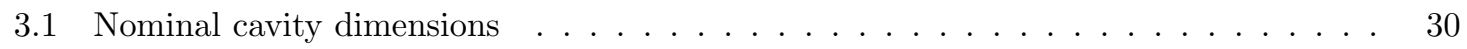

4.1 Phase fit example values . . . . . . . . . . . . . . . . . . . . . . . . . . . . . . . . . . 39

4.2 Al cavity, $\mathrm{T}=30^{\circ} \mathrm{C}$. . . . . . . . . . . . . . . . . . . . . . . . . . . . . . . . . . 44

$4.3 \mathrm{Au}$ cavity $\mathrm{TE}_{811}$ cleaning steps . . . . . . . . . . . . . . . . . . . . . . . . . . . . . 44

4.4 Uncertainties in $f_{0}$ and $Q$. . . . . . . . . . . . . . . . . . . . . . . . . . . . . . . 47

4.5 Measured cavity dimensions . . . . . . . . . . . . . . . . . . . . . . . . . . . 55

4.6 Measured vs. simulated of temperature change . . . . . . . . . . . . . . . . . . . . . 55

4.7 Measured and Simulated Tube $f_{0}$. . . . . . . . . . . . . . . . . . . . . . . . . . . . . 58

4.8 Methanol Results, $83 \mathrm{GHz}$. . . . . . . . . . . . . . . . . . . . . . . . . . . . . . . 67

4.9 Uncertainty budget for $\mathrm{TE}_{221}$ methanol data . . . . . . . . . . . . . . . . . . . . . . 70

4.10 Repeatbility of Methanol Results, $83 \mathrm{GHz}$. . . . . . . . . . . . . . . . . . . . . . . 71

C.1 Material properties for PCR studies . . . . . . . . . . . . . . . . . . . . . . . . . . 108

C.2 Debye formula parameters . . . . . . . . . . . . . . . . . . . . . . . . . . . . . . 110

C.3 PCR chamber impedance calculation . . . . . . . . . . . . . . . . . . . . . . . . . . . 111 


\title{
List of Symbols
}

\author{
basis/unit vector \\ A matrix \\ $\mu \quad$ permeability \\ $\omega \quad$ angular frequency \\ $\sigma \quad$ electrical conductivity \\ $\varepsilon \quad$ permittivity \\ $\varepsilon^{\prime \prime} \quad$ Imaginary part of permittivity \\ $\varepsilon^{\prime} \quad$ Real part of permittivity \\ $\varepsilon_{r} \quad$ Relative permittivity \\ $\vec{\square} \quad$ vector \\ $f_{0} \quad$ resonant frequency \\ $j \quad \sqrt{-1}$ \\ Q quality factor \\ dB decibel, e.g. $S_{21}(\mathrm{~dB})=20 \log _{10}\left(S_{21}\right)$ \\ TE Transverse Electric mode \\ TM Transverse Magnetic mode
}




\section{Chapter 1}

\section{Background}

Measurements of an electric field perturbed by a material can give insight into the charge distribution, or molecular structure, of the material. This principle is used in spectroscopy of liquids and biological molecules, which are both difficult to characterize solely by theoretical methods. The raw spectroscopy data is often transformed into a parameter, such as permittivity, that is more immediately relevant to the characterization of a material and is relatable among different measurement techniques. Permittivity data is also useful in and of itself for the creation of material references, which serve as a calibration aid for other measurement systems. Out of all liquids, the permittivity of water is of interest to many fields and consequently has been measured numerous times; however, its characterization is not complete. Conversely, design of permittivity measurement systems is guided by an expected electrical response of the sample under test; this is first ascertained by simplistic structural models. Thus, while molecular characterization is an end goal, it is still worthwhile to examine these physical models. In turn, mathematical expressions can be derived, giving estimates for the relative permittivity. Generally, the subdivision of matter (e.g. molecular or atomic) governs the frequency range of measurement, while the absolute permittivity values guide the sensitivity of the measurements.

Before going into the background, a brief note is made regarding the spectral unit, which varies among disciplines. In this thesis, the unit primarily used is Hertz, rather than inverse wavelength of $\mathrm{cm}^{-1} \equiv 30 \mathrm{GHz}$. Measurements were done in W-band, which spans $75-110 \mathrm{GHz}$, or $2.5-3.7 \mathrm{~cm}^{-1}$. 


\subsection{Physical Origin of Permittivity}

Within a material, charged bodies will move in response to the electromagnetic force of an applied electric field. The movement of these particles is not instantaneous as it is hindered by their masses and the bonds, if any, that hold them; this mathematically adds an imaginary component to permittivity, which is also referred to as loss. The particles vary in size, from sub-atomic to molecular scale. Thus it follows that each type of particle will react differently to the same oscillating field. This is what accounts for the dispersion of permittivity across the frequency spectrum. While there are many types of bodies that make up liquids, their behaviors can be mostly grouped into two different processes: orientational and distortional polarizations. Meanwhile, there are forces of friction, collisions, and random thermal effects that occur independently of these mechanisms.

Molecules that have permanent dipole moments will undergo orientational polarization, which is also known as relaxation. On average, molecules will reorient their dipole moments in accordance with the field polarity, as shown in Figure 1.1. As described above, dipole moments are dispersive. They easily follow the field at lower frequencies, but as the frequency increases, they struggle to maintain alignment due to their mass, and the lag between the polarization and the field increases; eventually at some frequency this lag reaches a local maximum. Beyond this frequency, the field alternates too quickly for the dipole moment, so the relaxation process has little effect on the permittivity. This type of loss mechanism is expected to occur from the microwave range into submillimeter-wave frequencies.

The permittivity of polar molecules was initially studied by Peter Debye. He created the Debye Model, which assumes an exponential decay form for the polarization when the applied field is turned off [1]. The following shows the permittivity of a material with a single relaxation:

$$
\varepsilon(\omega)=\varepsilon_{\infty}+\frac{\varepsilon_{s}-\varepsilon_{\infty}}{1+\jmath \omega \tau}
$$

where $\tau$ is the relaxation time, or the amount of time it takes for the polarization to decay by a factor of $1 / e$. This term is expected to depend on the mass and temperature of the dipole structure. The inverse of $\tau$ gives the frequency (in radians) at which the maximum loss occurs. The term $\varepsilon_{\infty}$ is the permittivity at infinite frequency, while $\varepsilon_{s}$ is the static permittivity. 

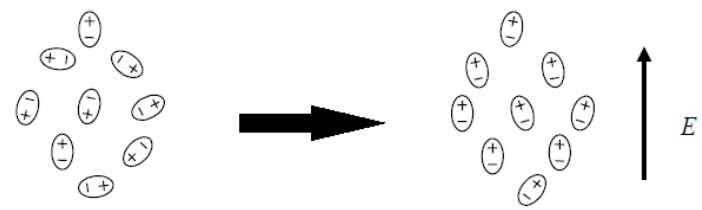

Figure 1.1: Illustration of orientational polarization. The image on the left shows the random orientation of dipoles and how they rotate in relation to the field, as seen on the right (from 2]).

At much higher frequencies, distortional polarizations occur, which affect the shape of the charge distribution. These are in the form of ionic and electronic polarizations. Examples of the former, also known as vibrational modes, are shown for the water molecule in Figure 1.2 Distortional polarizations are reminiscent of a damped harmonic oscillator; likewise each polarization will have its own natural frequency at which oscillatory behavior will reach a maximum, which are expected to lie in the terahertz region and above. Using the damped harmonic oscillator as a model, Herbert Frölich derived a term for these resonant polarizations, as shown in 1.2 , for a single resonance over the entire frequency spectrum. In this case, $\tau$ accounts for the damping and $\omega_{0}$ is the resonance frequency [1].

$$
\varepsilon(\omega)=\frac{\left(\varepsilon_{s}-\varepsilon_{\infty}\right)}{2}\left[\frac{1-\jmath \omega_{0} \tau}{1-\jmath\left(\omega_{0}+\omega\right) \tau}+\frac{1+\jmath \omega_{0} \tau}{1+\jmath\left(\omega_{0}-\omega\right) \tau}\right]
$$

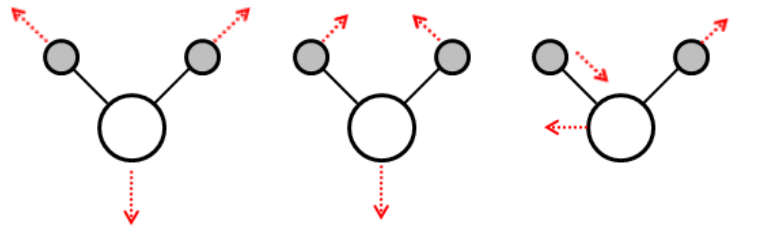

Figure 1.2: Illustration of vibrational modes for the water molecule. The dotted arrows indicate the initial direction of distortion for each atom.

When creating a permittivity function that covers the entire frequency spectrum, the polarization terms are summed, but with the $\varepsilon_{s}-\varepsilon_{\infty}$ term replaced with an intermediate term that roughly corresponds to the contribution of each particular mechanism to the overall dispersion. Initial estimates for $\tau$ and $\omega_{0}$ can be calculated from simple physical models. This leaves the permittivity at zero and infinite frequencies, which are usually estimated by measurements with a capacitance cell and the sodium D line, respectively [1]. In all, the dispersion of permittivity for a hypothetical 
material demonstrating three polarizations - orientational, ionic, and electronic - is shown in Figure 1.3. This graph also highlights the differences between the relaxation and resonance phenomena effects on permittivity. The real part will experience a gradual decrease for a relaxation, while it locally peaks for a resonance. As for the loss, the main difference is the width in the peak, with the relaxations producing a broader effect.

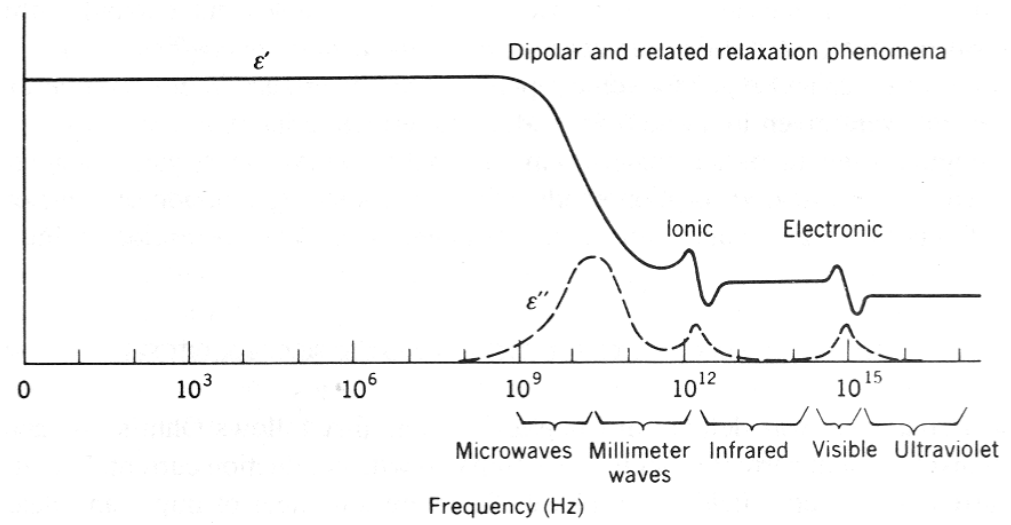

Figure 1.3: Broadband permittivity variation due to loss mechanisms (from $[3]$ ).

\subsection{Liquids}

Some forms of matter have a molecular structure that is so simplistic that its responsiveness to stimuli can be easily modeled. However, this is not the case with liquids, which are generally comprised of molecules that are highly structured yet still malleable in response to thermal or electrical sources 4]. Thus, experimental studies of liquids are done in conjunction with theoretical and computational methods.

There are a number of applications that require the knowledge of liquid permittivity, such as microwave dielectric heating, remote sensing, and moisture detection, among others [4]. These applications would greatly be served by the creation of multiple liquid standards, which would be used for instrument calibration or operational check [5]. There is a group at the National Physical Laboratory in the United Kingdom that is focused on the creation of highly precise liquid standards, but the work has been limited to below $10 \mathrm{GHz}$ 6]. Besides water, methanol and other alcohols have been measured by fewer studies for frequencies higher than $10 \mathrm{GHz}$ 7, 8. Including water, the alcohols 
are also good candidates as liquid standards because they are common solvents manufactured with high purity and are lossy, so they are more impervious to impurities [2].

\subsubsection{Water}

Above $100 \mathrm{GHz}$, there are a wide variety of applications for which water data is necessary, such as remote sensing and standoff detection. The characterization of biological molecules, which starts at $60 \mathrm{GHz}$, would be aided by the ability to measure molecules in their natural environment of an aqueous solution 9 10. However, the water obviously contributes to the spectroscopy measurements, so any effect due to water must be deconvolved [10]. Ultimately this data is gathered in order to create spectral signatures of chemical and biological warfare agents [11. Another use of water permittivity data is in modeling human skin in order to study standoff detection of weapons [12. Although water has been studied extensively, there remain discrepancies among the various studies at these frequencies.

In its purest form, water consists of two hydrogen atoms and one oxygen atom bonded covalently, which creates a permanent dipole between the hydrogen and the oxygen. Given its molecular structure, at least one dipolar relaxation is expected, along with the resonances at higher frequencies. In fact, all water studies have confirmed the first relaxation, occurring at approximately $19 \mathrm{GHz}$ for $25^{\circ} \mathrm{C}$. It is thought that this is the reorientation of a tetrahedral composition of water molecules $[13$. While this relaxation accounts for a majority of the dispersion, it is inadequate for modeling the data from $30 \mathrm{GHz}$ to $3 \mathrm{THz}$, above which the resonances become influential [14]. Most current models use at least two relaxations, the second one probably the relaxation of a single molecule 13 . However, the second one is ill-defined and has been characterized to occur from $150 \mathrm{GHz}$ to $900 \mathrm{GHz}$ [15]. This wide range appears to be a consequence of the number of terms chosen versus the measurement bandwidth, in that the second relaxation is overestimated when the maximum frequency is greater than $1 \mathrm{THz}$, as evidenced from studies by Kindt and Rønne 7.16. If three relaxations were used, or the data for two-relaxation models did not extend beyond $500 \mathrm{GHz}$, then the relaxation frequencies are much closer in agreement, ranging around $150 \mathrm{GHz}$ 14, 15, 18. This corresponds to an approximate 1 ps relaxation time and is in agreement with results from optical Kerr-effect 
spectroscopy methods, which do not measure permittivity but instead the transient response of a sample excited by a femotsecond laser 19,20 .

From the perspective of obtaining accurate permittivity data of water, however, the parameter values are not necessarily relevant as long as the model provides a good fit to the data. Still, there is a general lack of agreement among models at the higher end of the millimeter-wave range (mm-wave, 30-300 GHz, or 1-10 $\mathrm{cm}^{-1}$ ). Furthermore, from $100-300 \mathrm{GHz}$, not many measurements have been made of water 21].

\subsection{Measurement techniques}

Permittivity measurement methods exist from DC to optical frequencies and are based on frequencyspecific devices and equipment. Essentially the sample is introduced to a circuit so that it measurably alters the electric field. With a model of the circuit and sample, the permittivity can be extracted from the measurements.

In characterizing a material, the two main considerations are: at what frequencies is the permittivity to be measured, and what permittivity value magnitudes need be measured? There are many measurement methods, but the focus of this section will be on those that measured liquids in the millimeter-wave range or at its boundaries, or contributed to the characterization of water and other polar liquids. Specific studies with pertinent permittivity data are highlighted.

\subsubsection{Terahertz}

One feature of the "Terahertz gap" was a lack of bright single-frequency sources, which spurred the development of broadband-source spectroscopy. In particular, two types that have demonstrated operation at and below $100 \mathrm{GHz}$ are Terahertz Time-Domain Spectroscopy (THz-TDS) and Dispersive Fourier Transform Spectroscopy (DFTS). Both of these are quasi-optical techniques, meaning that free-space optical setups are used for wave transmission, but with wavelengths a few orders of magnitude larger than those of traditional optics. Recently, backward-wave oscillators (BWO) have emerged as a coherent source suitable for spectroscopy and have also been used for quasi-optical setups. In general, these techniques are not optimal at lower frequencies due to diffraction effects. 


\section{Terahertz Time-Domain Spectroscopy}

For Terahertz Time-Domain Spectroscopy (THz-TDS), a femtosecond-order laser pulse both creates and samples a THz pulse, via a photoconductive antenna and receiver, that has either been transmitted through or reflected from the sample. The Fourier Transform of the resulting waveform will give the response as a function of frequency. The lower frequency limit of THz-TDS is typically around $100 \mathrm{GHz}$, which results from lower efficiencies of the antenna and the mirrors used to direct the $\mathrm{THz}$ beam 22 . Temporal sampling of the $\mathrm{THz}$ waveform is done by a delay line, so the frequency resolution is set by the maximum delay and is typically $1 \mathrm{GHz} 23]$. This can be lowered with a greater delay, but with an increase in background noise which lowers the dynamic range of the system. The emitted $\mathrm{THz}$ radiation is sent through the liquid sample cell, although usually reflected off of the cell for better sensitivity, since the sample is lossy [24]. Both the real and imaginary parts of the permittivity can be found.

There have been a number of works that measured liquids with THz-TDS. Kindt measured and provided Debye fits for water and a number of alcohols from 60 to $1500 \mathrm{GHz}$. 7). Around the same time, Rønne measured water from 100 to $2000 \mathrm{GHz}$ at various temperatures [16. About a decade later, Jepsen measured water-ethanol mixtures from 100 to $1000 \mathrm{GHz}$, and also determined Debye parameters for water 25$]$.

\section{Fourier Transform Spectroscopy}

Fourier Transform Spectroscopy (FTS) systems are mainly used from $300 \mathrm{GHz}$ and up into terahertz frequencies. They are based on the Michelson Interferometer and rely on a broadband light source. DFTS is similar to conventional Fourier Transform Spectroscopy in that a wideband light source is split equally between a fixed and moving mirror, which create an interferogram in the spatial-domain. The main difference between conventional and DFTS systems is that the latter is phase-sensitive, so effectively the entire complex permittivity can be measured [24]. Similarly to THz-TDS, the frequency resolution is inversely proportional to the maximum distance traveled by the moving mirror. The lower frequency limit is set by a number of factors but mainly by the low output of the mercury lamp source below $300 \mathrm{GHz}$. For DFTS, much work was done to push this limit further below $300 \mathrm{GHz}$ by ensuring an optimal and stable setup with accurate modeling of optical 
components for improved SNR; while it has shown excellent accuracy and precision it remains to be a highly specialized technique $2,26,27$.

In 1977, Afsar measured water with DFTS from 0.15 to $13.5 \mathrm{THz} 28$. Vij used a DFTS system to measure water from 1.5 to $6.6 \mathrm{THz}$; this data, along with lower frequency literature data, was combined to produce a Debye fit 18 .

\subsubsection{Microwave}

Below $100 \mathrm{GHz}$, dielectric spectroscopy has been dominated by setups that include frequency-tunable sources and microwave components, which are mainly coaxial and waveguide. With highly accurate Vector Network Analyzers (VNA) providing high spectral resolution $(1 \mathrm{~Hz})$, these methods are more limited by the difficulty in scaling the components to higher frequencies. Measurement methods are split into broadband and resonant techniques, i.e. continuous vs. discrete frequencies.

\section{Broadband}

Coaxial techniques have mainly been used below $50 \mathrm{GHz}$ due to fabrication tolerances of coaxial components for single-moded operation. The most commonly used method is the coaxial probe. While it has shown operation up to $110 \mathrm{GHz}$ with a 1-mm coaxial probe, this method must be calibrated with a reference liquid. Other coaxial methods exist that do not need calibration, with the sample as either a semi-infinite load, short-circuited load, or section of the coaxial dielectric. The latter two are usually equipped with a variable-length cell for increased accuracy. However, these would be impractical with expensive 1-mm coax, which would have to be flushed of the sample; also the measurements become very sensitive to the coax and sample dimensions [5]. As part of the effort at NPL to obtain data for reference liquids, Gregory used a coaxial line to characterize methanol and other alcohols up to $5 \mathrm{GHz}[6$.

Above $50 \mathrm{GHz}$, rectangular waveguide is usually chosen. Many waveguide transmission/reflection techniques are similar to the coaxial methods in that the liquid sample is incorporated as a load or transmission cell whose surface area is equivalent to the cross-sectional area of the waveguide. As with coax, either moving parts or dielectric inserts are necessary to contain the sample, and thus their dimensions and physical placement must be characterized accurately unless proper calibration or 
modeling removes these effects. Barthel characterized water and some alcohols with Debye equations, using waveguide measurements up to $90 \mathrm{GHz} 29,30$. Concurrently, Richards measured water at $90 \mathrm{GHz}$ with an oversized waveguide system [31. In 1997, Duhamel measured methanol across several waveguide bands, up to $110 \mathrm{GHz}[32]$. More recently, Kouzai used advanced full-wave modeling techniques that allow for the use of a sample-containing tube placed in the waveguide, for up to $50 \mathrm{GHz}[33$.

Similar to the terahertz methods, there are free-field setups that use horn antennas to transmit the signal to the sample. These methods are most suitable for large, planar samples so that planewave approximations can be used, or Gaussian Beam approximations if focussing elements are used. Even with the latter technique, the sample size must still be at least six times the wavelength 2 .

\section{Resonant}

A resonant circuit can easily be made out of any type of mm-wave transmission line. Naturally these methods only give results at one or a few frequencies, but they are used for their high sensitivity. As a result, resonators are more renowned for the measurement of low-loss dielectrics. However, lossier samples can still be measured provided that not so much of the power is absorbed that the fields cannot build up to resonance. This can be done by either using a small sample or placing the sample in a region of the cavity where the electric field is weak.

Fabry-Perot cavities have the largest quality factors of 100,000 and higher; this is a free-space method, so again the sample must be much larger than the beam width.

Whispering gallery mode dielectric resonators have recently been developed. The "whispering gallery" describes a mode pattern in which the field lobes are at the edges of the resonator. Evanescent fields existing just outside of the resonator interact with the liquid sample. At $35 \mathrm{GHz}$, Eremenko measured water-alcohol solutions using a hemispherical dielectric resonator set at the bottom of a container and immersed in the liquid sample 34. Above $100 \mathrm{GHz}$, Shaforost demonstrated use of a whispering gallery resonator for liquid measurements, albeit for a sensing application 35.

Waveguide cavities offer lower Q-factors than their open-cavity Fabry-Perot counterparts, but they have been used more for liquid measurements for the smaller sample volumes. In general, studies that used cavity resonators were limited to frequencies below $100 \mathrm{GHz}$ because only the 
fundamental mode was used. However, waveguide is commonly used above this frequency, can be easily modeled, and can provide high Q-factors, so it was chosen for precision measurements of liquids. The next chapter will go into more detail about waveguide cavities.

\subsection{Conclusion and Thesis Overview}

The background of liquid permittivity and the importance of its determination were discussed. Around $100 \mathrm{GHz}$ there is less data than at other frequency bands due to the instrumentation. In order to supplement the scarce higher frequency data for molecular characterization and the creation of liquid standards, a measurement setup using a waveguide cavity was chosen.

The next chapter will provide more detail on cavity resonators and their use for dielectric measurements, and then describe the modeling of the liquid-loaded cavity. In Chapter 3 , the design of the waveguide resonator for precision measurements is presented, and Chapter 4 analyzes the results the performance of the rigourously-modeled resonator system for permittivity measurements. 


\section{Chapter 2}

\section{Cavity Design and Modeling}

With every permittivity method, there is a model that relates the measurable parameters to the underlying permittivity of the sample. This chapter first describes the relevant physical and dimensional properties of the cavity and then lays out the modeling to solve for the permittivity from measurements.

\subsection{Background}

Before presenting the modeling for the measurement system, a preliminary background and rationale will be given of the method.

\subsubsection{Circular cavity}

A circular cavity is essentially a right circular cylinder, made of metallic walls, of radius $R$ and closed off at both ends so that its length is $h$, as seen in Figure 2.1. The multiple modes that can resonate in this structure are found by solving the boundary conditions for the electric and magnetic fields, which are governed by the Helmholtz equation. Assuming the walls are made from perfect electric conductors, an adequate approximation for high conductivity metals, the electric field boundary 
conditions of this structure result in the following equation for the resonant frequencies 36 :

$$
f_{0_{m n p}}=\frac{1}{2 \pi \sqrt{\mu \varepsilon}} \sqrt{\left(\frac{\chi_{m n}}{R}\right)^{2}+\left(\frac{p \pi}{h}\right)^{2}}
$$

where $m, n$, and $p$ are integers, $\mu$ and $\varepsilon$ are respectively the permeability and the permittivity of the material filling the cavity, and $\chi_{m n}$ is the $n$th zero of either the Bessel function or its derivative of the first kind of order $m$. Modes are classified into two categories, either Transverse Electric (TE) or Transverse Magnetic (TM), signifying that there is respectively no electric or magnetic component of the field in the axial direction. For both types, the $m, n$, and $p$ indices further define a unique mode, such as the $\mathrm{TE}_{111}$ and $\mathrm{TM}_{010}$ modes; each index also signifies the field variation in $\hat{\phi}, \hat{\rho}$, and $\hat{z}$.

The resonant frequency is only one measurable piece of data, so it alone is not sufficient for characterizing both the real and imaginary parts of the permittivity. In addition there is the quality factor $Q$, which is the ratio of the average stored energy to the dissipated power. Two sources of electric loss exist: the conductor loss of the cavity walls and the dielectric loss of the filling medium; if the former is known, then both components of the permittivity can be found. For a closed cavity, there are also closed analytical expressions for the quality factor 37 .

However, a closed cylinder completely filled with liquid is not practical for measurements. First, polar liquids at higher millimeter-wave frequencies are still quite lossy, so in order to observe a not completely damped response it is necessary to have more control of the filling factor by, say, only adding a small amount of sample to the cavity [5]. Second, the cavity can never be completely closed because an excitation mechanism is needed to measure the response. Both the inhomogeneous filling and the external excitation will perturb the ideal resonant frequencies and quality factors. To account for these modifications, various resonant methods have been developed, but the following short survey will mainly focus on those used to measure liquids.

\subsubsection{Resonant methods}

Cavity resonators were initially used with the cavity perturbation method. Similarly to general perturbation theory, the permittivity is found by relating it to the difference in the responses of the 


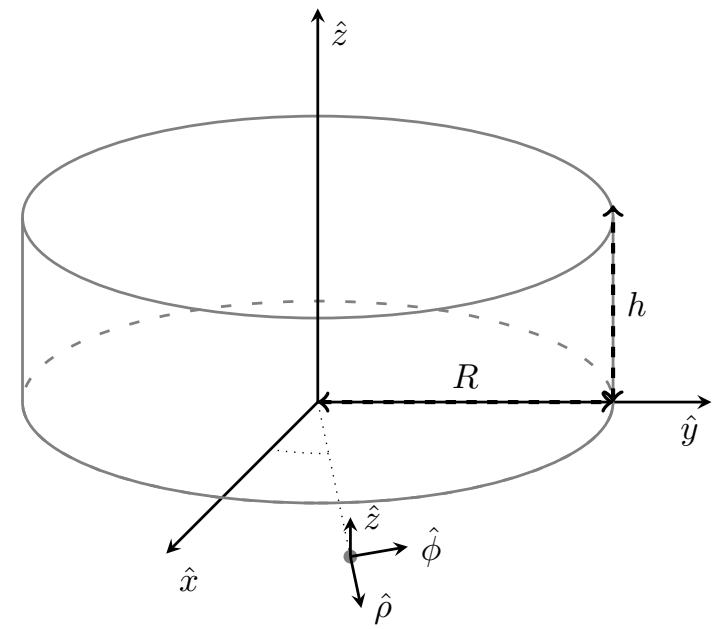

Figure 2.1: Circular cavity within the cylindrical coordinate system.

original and perturbed cavity with the sample. Bethe and Schwinger are credited with developing this method, and Birnbaum and Franeau used it to measure liquids 38 40. Generally, the fields of the perturbed cavity are approximated as being equivalent to those of the unloaded cavity. This requires that the sample be small, and limits measurement to modes that are well-isolated in frequency from adjacent modes, so typically the fundamental mode. The reduced computation of this method was especially beneficial to the measurement of liquids, which require a container and also an opening in the cavity through which they can be inserted; both of these experimental considerations further alter the ideal cavity case. Of course, the cavity itself can be used as the container if low-loss liquids are measured; in 1973, Stumper configured a cavity that was partially filled with hydrocarbon liquids, whose permittivity was found by modeling the fields 41.

Faster computers, though, facilitated more rigorous modeling that allowed for containers and insertion holes to be modeled. Li, Yu, and Kawabata used field-matching techniques for the measurement of liquids using the $\mathrm{TM}_{010}$ mode 42 44. This is a fundamental mode with strong fields in the center of the cavity, so the amount of liquid measured was limited to less than 1 percent of the total cavity volume. Since it is computationally easier to keep the liquid in the center of the cavity, other resonant modes have been explored, such as by Krupka or Regier, who used the $\mathrm{TE}_{01 \delta}$ modes, which have a field null in the center [45,46].

While the fields were modeled, most of the above methods were mainly used for only one resonant 
mode, thus providing permittivity at only a single frequency (Krupka provided data for two resonant modes). Within the past decade, though, multi-moded cavity resonator techniques have been developed, albeit for the measurement of solid samples. A split-cylinder resonator was created by Janezic for measurement of low-loss substrates, which was capable of measuring permittivity at seven frequencies across a $15 \mathrm{GHz}$ band starting at $10 \mathrm{GHz}$; both $\mathrm{TE}_{0 n p}$ and $\mathrm{TE}_{2 n p}$ modes were used [4]. Cheng developed a similar method for measuring low-loss solids at several resonant frequencies of $\mathrm{TM}_{0 n p}$ modes in a $10 \mathrm{GHz}$ band 48 . Both of these systems used a characteristic equation that assumed a closed cavity, and then either neglected the coupling effects because of low coupling or used a circuit model. Another method, by Shan, incorporated the coupling effects into the modeling, using eight $\mathrm{TM}_{0 n 0}$ modes over a $70 \mathrm{GHz}$ band 49 ]. While more computationally intensive, modeling of the coupling is more accurate as it does not necessitate a circuit model or low coupling for valid computation of the quality factor, whose value determines the imaginary permittivity of the sample yet will change with higher coupling. In Janezic's work, the coupling is at approximately $-50 \mathrm{~dB}$, which is low enough that the measured $Q$ is considered equivalent to the unloaded $Q$, to which his modeling was compared. This low coupling works at frequencies below $50 \mathrm{GHz}$ and for measuring low-loss samples, but is not feasible for measuring lossy liquids with the smaller dynamic range of the available W-band VNA system (about $75 \mathrm{~dB}$ ). Higher coupling and a circuit model was used in Cheng's work, but only modes of a certain azimuthal variation were excited and the circuit model assumed that modes were well-isolated from one another, which places limitations on the cavity setup.

With full-wave modeling, it is possible to use higher order modes and measure at multiple frequencies. It also provides more options for electric fields variations and interactions with samples.

\section{$2.2 \quad$ Full-wave modeling}

A simplified diagram of the over-moded cavity system is shown in Figure 2.2. A circular cavity is coupled to input and output waveguides via apertures, while a hole in the center of the cavity allows for the insertion of the tube. The rationale for this specific configuration is explained in more 


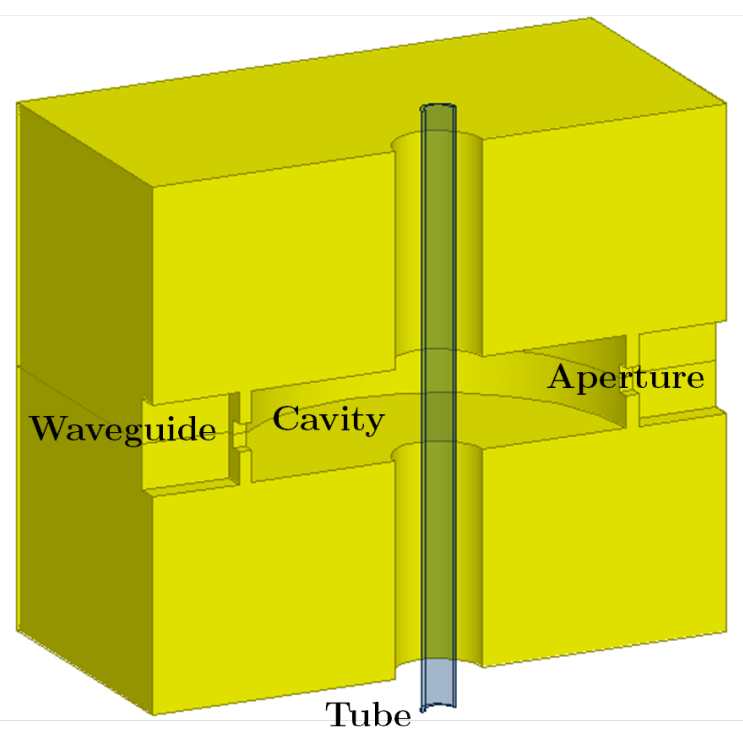

Figure 2.2: Cross-sectional view of over-moded cavity block and tube for permittivity measurements

detail in 3.1.1. Otherwise, the setup is also similar to previous techniques [43, 44]. In short, it simplifies the modeling and manufacture of the cavity. Based on the geometry of the cavity setup, a suitable modeling method was chosen. The finite element and finite difference methods are general techniques that can mesh arbitrarily-shaped volumes. While commonly used, these methods are not good for electrically large structures, such as an over-moded cavity.

\subsubsection{Mode-matching}

Looking at Figure 2.2, it can be seen that the geometry of the structure consists of shapes that are easily characterized by the common Cartesian and cylindrical coordinate systems. Indeed, the system can be subdivided into rectangular and circular waveguides, for which there are eigenmode solutions to the Helmholtz equation. This is the basis of the mode-matching method, which finds the superposition of these modes that will satisfy the boundary conditions. The mode-matching method is commonly used for the modeling of waveguide filters, T-junctions, couplers, dielectric resonators, and horn antennas 5054 .

Generally speaking, the contribution of each mode is found by matching both the electric and magnetic fields at a discontinuity, hence "mode-matching". In the literature this technique has also 
been described as the conservation of complex power or reaction, or modal analysis [55 57. At a discontinuity, all of these methods apply a cross-product operation between the electric and magnetic fields that results in a function which is analytically (or nearly) integrable over a finite surface area.

The problem is set up by subdividing the structure, either by a change in geometry or material, so that at each discontinuity the only unknowns are the normal mode coefficients. A simple discontinuity is shown in Figure 2.3 of waveguides 1 and 2, whose respective cross-sections of $S_{1}$ and $S_{2}$ differ in size but share an equivalent longitudinal axis $\hat{n}$. In each region, the electric and magnetic fields are approximated as a sum of modes, where the subscript $T$ denotes the field tangential to $\hat{n}$, and $A_{1,2}^{l, m}$ and $B_{1,2}^{l, m}$ are the incoming and outgoing mode coefficients, respectively

$$
\begin{array}{ll}
\vec{E}_{1_{T}} \cong \sum_{l=1}^{L} \vec{e}_{1}^{l}\left(B_{1}^{l}+A_{1}^{l}\right) & \vec{E}_{2_{T}} \cong \sum_{m=1}^{M} \vec{e}_{2}^{m}\left(A_{2}^{m}+B_{2}^{m}\right) \\
\vec{H}_{1_{T}} \cong \sum_{l=1}^{L} \vec{h}_{1}^{l}\left(B_{1}^{l}-A_{1}^{l}\right) & \vec{H}_{2_{T}} \cong \sum_{m=1}^{M} \vec{h}_{2}^{m}\left(A_{2}^{m}-B_{2}^{m}\right)
\end{array}
$$

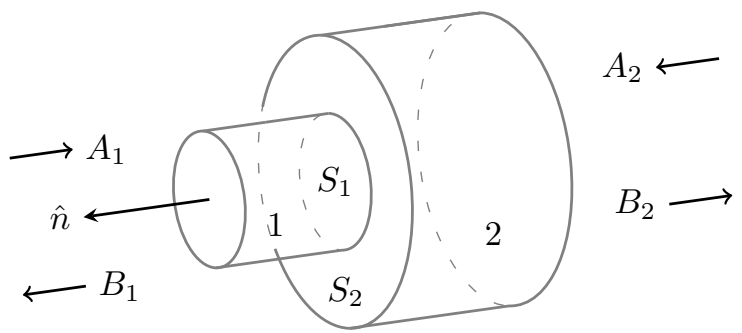

Figure 2.3: Diagram of waveguide discontinuity for mode-matching method.

At the discontinuity, the following boundary conditions are enforced

$$
\begin{aligned}
& \vec{E}_{2_{T}}= \begin{cases}\vec{E}_{1_{T}} & \text { on } S_{1} \\
Z_{s} \vec{J}_{s} \simeq Z_{s}\left(-\hat{n} \times \vec{H}_{2_{T}}\right) & \text { on } S_{2}-S_{1}\end{cases} \\
& \vec{H}_{1_{T}}=\vec{H}_{2_{T}} \text { on } S_{1}
\end{aligned}
$$

where 55

$$
Z_{s} \simeq(1+j) \sqrt{\frac{\omega \mu}{2 \sigma}} \text { for } \sigma \gg 0
$$


Usually an infinite conductivity is assumed. To satisfy the boundary conditions, cross-products of the following form are taken

$$
\langle\vec{a}, \vec{c}\rangle=\int_{S}(\vec{a} \times \vec{c}) \cdot \hat{n} \cdot d \vec{S}
$$

where in literature dealing with mode-matching, this cross-product is also referred to as an inner product between the field being matched and a weighting function $54,58,59$. As in 2.2 , the fields are approximated as a superposition of modes, or basis functions. Since the weighting function is the dual of the field being matched, these cross-products reduce to definite integrals of orthogonal bases, similarly to a generalized Fourier series.

For the electric fields, the magnetic field modes of the larger region are used for the weighting functions, as this enforces condition 2.3a 56

$$
\begin{aligned}
\left\langle\vec{E}_{1_{T}}, \vec{h}_{2}^{m}\right\rangle & =\left\langle\vec{E}_{2_{T}}, \vec{h}_{2}^{m}\right\rangle \\
\left\langle\sum_{l=1}^{L} \vec{e}_{1}^{l}\left(B_{1}^{l}+A_{1}^{l}\right), \vec{h}_{2}^{m}\right\rangle & =\left\langle\sum_{m=1}^{M} \vec{e}_{2}^{m}\left(A_{2}^{m}+B_{2}^{m}\right), \vec{h}_{2}^{m}\right\rangle
\end{aligned}
$$

This results in a system of $M$ linear equations, which are set up in matrix form

$$
\begin{aligned}
{\left[\begin{array}{ccc}
\left\langle\overrightarrow{e_{1}^{1}}, \overrightarrow{h_{2}^{1}}\right\rangle & \cdots & \left\langle\overrightarrow{e_{1}^{L}}, \overrightarrow{h_{2}^{1}}\right\rangle \\
\vdots & \ddots & \vdots \\
\left\langle\overrightarrow{e_{1}^{1}}, h_{2}^{M}\right\rangle & \cdots & \left\langle\overrightarrow{e_{1}^{L}}, h_{2}^{M}\right\rangle
\end{array}\right]\left[\begin{array}{c}
B_{1}^{1}+A_{1}^{1} \\
\vdots \\
B_{1}^{L}+A_{1}^{L}
\end{array}\right]=\left[\begin{array}{ccc}
\left\langle\overrightarrow{e_{2}^{1}}, \overrightarrow{h_{2}^{1}}\right\rangle & \cdots & \left\langle e_{2}^{\vec{M}}, \overrightarrow{h_{2}^{1}}\right\rangle \\
\vdots & \ddots & \vdots \\
\left\langle\overrightarrow{e_{2}^{1}}, \overrightarrow{h_{2}^{M}}\right\rangle & \cdots & \left\langle e_{2}^{\vec{M}}, h_{2}^{\vec{M}}\right\rangle
\end{array}\right]\left[\begin{array}{c}
A_{2}^{1}+B_{2}^{1} \\
\vdots \\
A_{2}^{M}+B_{2}^{M}
\end{array}\right] } \\
\mathbf{\Phi}\left[B_{1}+A_{1}\right]=\boldsymbol{\Psi}_{\mathbf{2}}\left[A_{2}+B_{2}\right]
\end{aligned}
$$

If the medium of Region 2 is lossless and the modes are normalized, then due to modal orthogonality, $\mathbf{\Psi}_{\mathbf{2}}$ will reduce to an identity matrix. This procedure is similarly done for the $\mathrm{H}$-field boundary condition, with the electric field modes of Region 1 used as the weighting functions; these provide an additional $L$ equations, which is sufficient [56] - presumably, the incoming waves are known, so the $L+M B_{1}$ and $B_{2}$ coefficients are the unknowns. Also by using the Region 1 electric field modes, 
this enables the matrix transpose of $\mathbf{\Phi}$ to be used, reducing the number of computations.

$$
\mathbf{\Psi}_{\mathbf{1}}\left[B_{1}-A_{1}\right]=\boldsymbol{\Phi}^{\mathbf{T}}\left[A_{2}-B_{2}\right]
$$

For multiple discontinuities matrix algebra is performed, substituting unknown mode coefficients with the equivalent formula of inner product matrices until the final region is reached, where a mode coefficient can be defined by a known excitation. For instance, in 2.6 and 2.7), if the incoming waves $A_{1}$ and $A_{2}$ are known, then $B_{1}$ and $B_{2}$ can be found. This is basically the analysis in Shan [49].

This procedure can also be used for regions with standing waves. If the final region is definable by a known boundary, such as a perfect electric conductor, then 2.6 becomes

$$
\Upsilon C=0
$$

which is a homogeneous system of linear equations. The mode coefficients $C$ are the non-trivial solutions, which is the nullspace of $\boldsymbol{\Upsilon}$. This also corresponds to a zero-determinant, and typically in electromagnetic problems the resonant frequencies are found from a root-search of the determinant. Both Janezic and Cheng used this method 47,48.

\subsubsection{Modal indices}

For the sake of clarity, a brief discussion of what constitutes a mode is included here. In waveguide, with a closed transverse area and an indefinite longitudinal length like either regions 1 or 2 in Figure 2.3 a mode is typically TE or TM, as described in 2.1.1 A specific TE- or TM-mode is further designated by two indices, unlike the three indices for the modes of the circular cavity, which is closed in all three dimensions; again, each index corresponds with a dimension. However, for the modeling analysis, these two indices are collapsed into a single index.

\subsubsection{Generalized Scattering Matrix}

Instead of solving for the mode coefficients, a more prudent formulation of the Generalized Scattering Matrix (GSM) is introduced, as it is both immediately comparable to data provided by the network analyzer and also facilitates the analysis of multiple discontinuities with both incoming and outgoing 
waves, which can be seen in A.7. Essentially, the outgoing waves are related to the incoming waves by scattering parameter relations

$$
\begin{aligned}
& B_{1}=\mathbf{S}_{\mathbf{1 1}} A_{1}+\mathbf{S}_{\mathbf{1 2}} A_{2} \\
& B_{2}=\mathbf{S}_{\mathbf{2 1}} A_{1}+\mathbf{S}_{\mathbf{2 2}} A_{2}
\end{aligned}
$$

which bear a strong resemblance to those of a conventional two-port $S$-parameter matrix. The difference is that the GSM includes scattering coefficients for more than a single mode, including evanescent ones.

To obtain the GSM for the discontinuity in Figure 2.3, 2.9a and 2.9b are substituted into the boundary condition equations. Starting with 2.6

$$
\mathbf{\Phi}\left[\left(\mathbf{S}_{\mathbf{1 1}}+\mathbf{I}\right) A_{1}+\mathbf{S}_{\mathbf{1 2}} A_{2}\right]=\mathbf{\Psi}_{\mathbf{2}}\left[\mathbf{S}_{\mathbf{2 1}} A_{1}+\left(\mathbf{I}+\mathbf{S}_{\mathbf{2 2}}\right) A_{2}\right]
$$

The factors of $A_{1}$ and $A_{2}$ on both sides are set equal to one another, and since $\boldsymbol{\Phi}$ is likely not to be a square matrix and thus cannot be inverted, the following relations are found, in which $\mathbf{I}$ is the identity matrix

$$
\begin{aligned}
& \mathbf{S}_{21}=\boldsymbol{\Psi}_{\mathbf{2}}^{-1} \boldsymbol{\Phi}\left(\mathbf{S}_{11}+\mathbf{I}\right) \\
& \mathbf{S}_{\mathbf{2 2}}=\boldsymbol{\Psi}_{\mathbf{2}}^{-1}\left(\boldsymbol{\Phi} \mathbf{S}_{12}-\mathbf{\Psi}_{\mathbf{2}}\right)
\end{aligned}
$$

This is repeated for the magnetic field boundary condition 2.7), with the additional step of substituting in 2.10 and 2.11, resulting in

$$
\begin{aligned}
& \mathbf{S}_{\mathbf{1 1}}=\left(\boldsymbol{\Psi}_{\mathbf{1}}+\boldsymbol{\Psi}_{\mathbf{2}}{ }^{-1} \boldsymbol{\Phi}\right)\left(\boldsymbol{\Psi}_{\mathbf{1}}-\boldsymbol{\Psi}_{\mathbf{2}}{ }^{-1} \boldsymbol{\Phi}\right) \\
& \mathbf{S}_{\mathbf{1 2}}=2\left(\boldsymbol{\Psi}_{\mathbf{1}}+\boldsymbol{\Phi}^{T} \boldsymbol{\Psi}_{\mathbf{2}}{ }^{-1} \boldsymbol{\Phi}\right)^{-1} \boldsymbol{\Phi}^{T}
\end{aligned}
$$




\subsection{Modeling of four port aperture-coupled cavity junction}

The relevant aspects for modeling the cavity for liquid measurement are shown in Figure 2.4. In order to include possible radiation effects at the end of the hole containing the tube, the structure is modeled as a four-port junction, after Zheng [59]. Other mode-matching analyses of a sidewall aperture-coupled cavity have been done by Melloni and Rong, although the cavities in both of these papers were closed except for the aperture $[58,60]$. In Kawabata's work of an eigenfrequency analysis of a concentric liquid-tube configuration in a $\mathrm{TM}_{010}$ cavity, the ends of the hole were shorted with both electric and magnetic walls, and the hole length in the modeling was increased until the resonant frequency for both types of walls matched 44 .

The modeling was coded in MATLAB ${ }^{\circledR}$, for its availability and ease of use, particularly with matrices 1

\subsubsection{Boundary conditions}

Looking at Figure 2.4. Regions $W$ and $A$ are respectively the waveguide and aperture regions, both rectangular cross-sections. Regions $C$ and $H$ are inhomogeneous circular waveguide. In the center, $V$ and $P$ are intermediate regions, where $V$ is electrically shorted at $\rho=R_{c}$ and $P$ is likewise shorted at $z= \pm \frac{z_{0}}{2}$. These intermediate regions are necessary because the waveguide and insertion hole are in different directions, so if a single wavefunction were to be formulated for the cavity region, the transverse functions could not be completely defined at the waveguide and insertion hole discontinuities. As discussed in $\$ 2.2 .1$, the normal mode coefficients need to be the only unknowns at a discontinuity. By creating these regions with artificial boundaries, the fields can be formulated for the mode-matching technique. The fields for all of the regions are explicitly derived in Appendix A It was decided to automatically include the conductor loss of the cavity in the analysis. On the cavity wall of the $P$-region this was accomplished in the mode-matching analysis, as in 2.3a). For the walls of Regions $C$ and $H$, the loss is included in the modal propagation constants, which are found numerically (for lossless, homogeneous waveguide, these propagation constants are simply related to the Bessel zeros).

\footnotetext{
${ }^{1}$ The MathWorks Inc., MATLAB, Natick, MA
} 


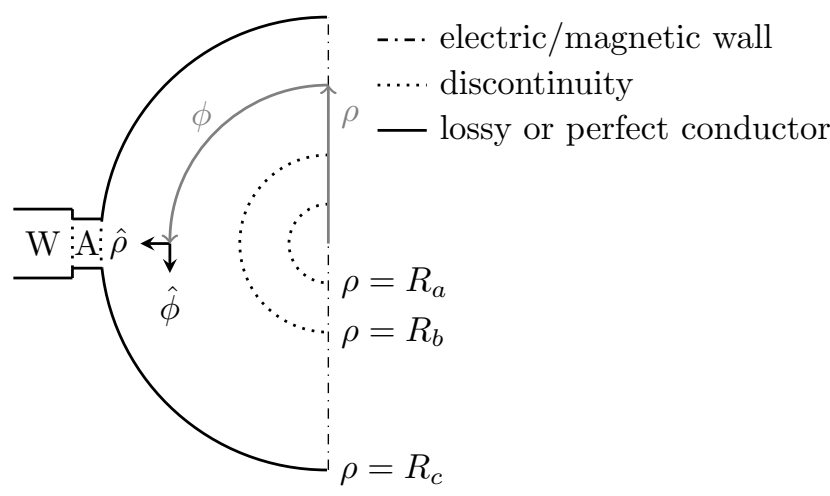

(a) Cross-section, $z=0$

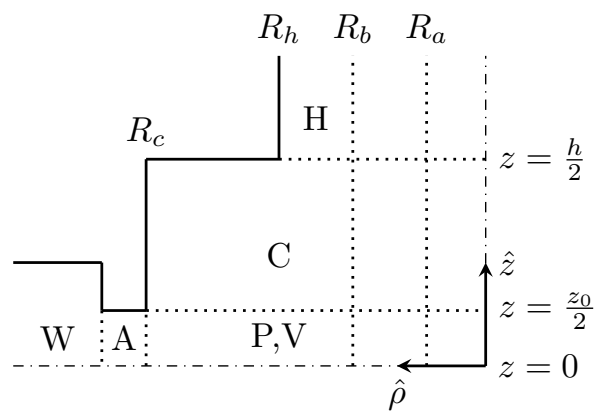

(b) Cross-section, $\phi=\frac{\pi}{2}$

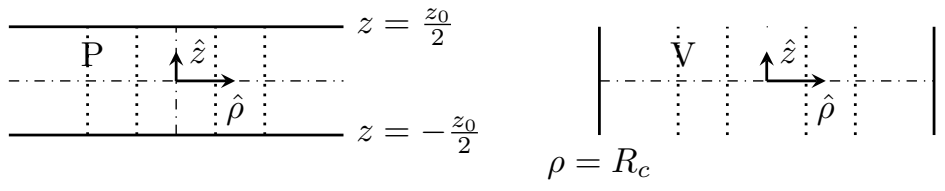

(c) $P$ - and $V$ - intermediate regions for junction model. $P$ is radial waveguide with perfect conductors at $z=$ $\pm \frac{z_{0}}{2}$, while $V$ is inhomogeneous circular waveguide with perfect conductor at $\rho=R_{c}$.

Figure 2.4: Diagram of regions and discontinuities for modeling.

As the structure is symmetric, only a quarter of it needs to be analyzed, and the number of discontinuities in the problem can be reduced; the "electric/magnetic wall" lines in Figure 2.4 denote the planes of symmetry. Additionally, this structure is not modeled as a whole, but is divided into multiple $S$-matrices, as shown in Figure 2.5, which are subsequently combined. The $S$-matrices $S_{W}$ and $S_{H}$ are found as in $\$ 2.2 .1$ and $\$ 2.2 .3$ whereas $S_{A}$ and $S_{C}$ are simply diagonal matrices that represent a phase shift in the waves resulting from traveling through a section of uniform waveguide. In the following analysis, the boundary conditions that result in $S_{J}$ are presented. Derivation of the final $S$-parameters for the whole structure can be found in A.8. 
At $z=\frac{z_{0}}{2}$, enforcement of the boundary conditions produces

$$
\begin{aligned}
\hat{z} \times \vec{E}_{C} & =\hat{z} \times \vec{E}_{V} \\
\left\langle\vec{e}_{C}, \vec{h}_{V}\right\rangle\left(B_{C}+A_{C}\right) & =\left\langle\vec{e}_{V}, \vec{h}_{V}\right\rangle A_{V} \\
\hat{z} \times \vec{H}_{C} & =\hat{z} \times\left(\vec{H}_{V}+\vec{H}_{P}\right) \\
\left\langle\vec{e}_{C}, \vec{h}_{C}\right\rangle\left(B_{C}-A_{C}\right) & =\left\langle\vec{e}_{C}, \vec{h}_{V}\right\rangle A_{V}+\left\langle\vec{e}_{C}, \vec{h}_{P}\right\rangle A_{P}
\end{aligned}
$$

At $\rho=R_{c}$, the boundary conditions are:

$$
\begin{aligned}
\hat{\rho} \times \vec{E}_{P} & = \begin{cases}\hat{\rho} \times \vec{E}_{A} & \text { on } S_{A} \\
Z_{s}(-\hat{\rho}) \times\left(\vec{H}_{V}+\vec{H}_{P}\right) & \text { on } S_{P}-S_{A}\end{cases} \\
{\left[\left\langle\vec{e}_{P}, \vec{h}_{P}\right\rangle+Z_{s}\left\langle\left(\hat{\rho} \times \vec{h}_{P}\right), \vec{h}_{P}\right\rangle\right] A_{P} } & \\
+Z_{s}\left\langle\left(\hat{\rho} \times \vec{h}_{V}\right), \vec{h}_{P}\right\rangle A_{V} & =\left\langle\vec{e}_{A}, \vec{h}_{P}\right\rangle\left(B_{A}+A_{A}\right) \\
\hat{\rho} \times\left(\vec{H}_{P}+\vec{H}_{V}\right) & =\hat{\rho} \times \vec{H}_{A} \\
\left\langle\vec{e}_{A}, \vec{h}_{P}\right\rangle A_{P}+\left\langle\vec{e}_{A}, \vec{h}_{V}\right\rangle A_{V} & =\left\langle\vec{e}_{A}, \vec{h}_{A}\right\rangle\left(B_{A}-A_{A}\right)
\end{aligned}
$$

From the electric boundary conditions 2.14a and 2.15a, $A_{V}$ and $A_{P}$ are found in terms of the scattering parameters $A_{A}, A_{C}, B_{A}$, and $B_{C}$, then substituted into the magnetic boundary conditions 2.14b) and 2.15b). In a similar fashion to the procedure in 2.2 .1 and $2.2 .3 . S_{J}$ can be determined.

It should be noted that the cross-products in 2.14 and 2.15 are merely reactions, i.e. the complex conjugate is not taken on one of the arguments. However, because lossy materials are included, these cross-products are strictly speaking not inner products as they violate the Hermitian transpose property. Rather, they are of the form of a "pseudo inner product" [61, which was simpler to implement in code. In the mode-matching literature, some authors use the complex conjugate while others simply stick with the reaction, although the latter take steps to ensure that their functions are real-valued by changing basis functions, e.g. switching to hyperbolic sine if the 
argument is imaginary, though the rationale for this is not explained. This was of some concern, so a simple case of two waveguides coupled through an aperture, with each region filled with a lossy dielectric was tested. The inner product and the pseudo inner product gave equivalent $S$-parameters for the $\mathrm{TE}_{10}$ mode.

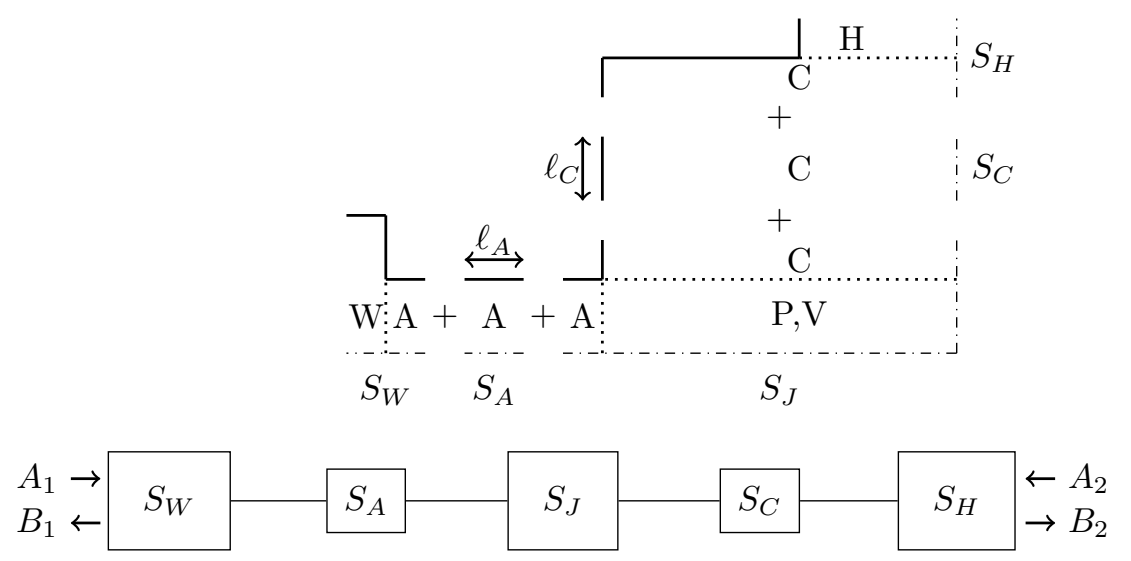

Figure 2.5: Transformation of discontinuities into cascaded $S$-matrices.

\subsubsection{Inhomogeneous propagation constants}

When dielectrics are added to the cylindrical waveguide regions $V, C$, and $H$, the wavenumbers cannot be defined in a straightforward manner as with the single boundary condition on the empty waveguide wall. The boundary conditions between the dissimilar dielectrics must be satisfied. This is done in a manner very similar to Yeh's work, in which the boundary conditions are formulated into multiple matrix equations, one for each discontinuity, instead of a single matrix that includes equations for the whole structure 62 . In this way, the smaller matrices are combined into a final $2 \mathrm{x} 2$ matrix, for which the determinant is easier to compute than for a 10x10 matrix, i.e. two coefficients in the center region and four coefficients each in the outer two regions.

The determinant of the $2 \times 2$ matrix is the characteristic equation whose roots are the propagation constants. Because of the lossy dielectrics and outer conductor, the root search must be over a complex region. The Lehmer-Schur algorithm was chosen for its ease of implementation; it is essentially the two-dimensional version of a bracket search [63. The root-search algorithm proceeds by first determining whether there is a root in a region. This is done by computing the argument 
principle 64,

$$
\oint_{C} \frac{f^{\prime}(z)}{f(z)} d z=2 \pi i\left(N_{Z}-N_{P}\right)
$$

where $C$ is a closed curve that defines the region, $f(z)$ is the function for which the roots are desired, $N_{Z}$ is the number of zeros in the region, and $N_{P}$ is the number of poles, of which there should be none. This is done numerically with Henrici's algorithm by accumulating the change in argument of the function along the closed curve 65]. If $N_{z}>1$, this means that there is more than one root, and the region is divided into quarters which slightly overlap so as to avoid a zero coinciding with the closed curve 66, 67. This recursive process continues until a single root has been found within a region of a precision that is suitable for providing an estimate of the zero, and the center of this region is returned. A more accurate value of the root is then obtained using Muller's method, which is capable of handling complex roots 63. If the region providing the initial guess is too large, then Muller's method could converge to a neighboring zero, and the true zero would be lost.

Zeros were searched for in the fourth quadrant, over subregions that excluded trivial solutions to the characteristic equation, as seen in Figure 2.6. In testing the method, it was found that the Bessel zeros were adequate as initial guesses for the Muller's method step for both lossy waveguide that was either empty or contained the quartz tube, since both cases only slightly perturb the lossless empty waveguide case. Thus, the complex root search algorithm was used only to find roots for when a liquid is in the tube, and also to find the zeros that cannot be predicted by the Bessel zeros, i.e. normalized propagation constants greater than 1, which occur for inhomogeneous waveguide.

\subsubsection{Bessel conditioning}

A few extra steps were taken with handling Bessel function arguments for the $P$-region modes. First, for all modes that had purely imaginary arguments, an extra factor was inserted into the modal coefficients so that the arguments could be made real, with the modified Bessel functions replacing the ordinary ones. This change was based on previous work 58. While the rationale for this was not explicitly stated, it was likely done because the modified functions are the evanescent form of the ordinary functions, i.e. they are exponential and not oscillatory. However, it was also found useful in MATLAB since the internal Bessel functions returned complex results, albeit with $\mathrm{a} \sim 10^{-16}$ real part, for a purely imaginary argument. 


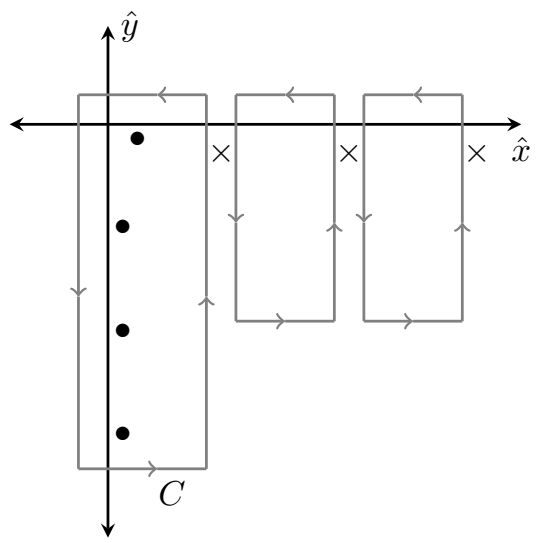

Figure 2.6: Example diagram of complex root search, particularly for a liquid-filled tube. Only the two left regions are searched for the empty tube, and the leftmost region for the empty cavity. In region $C$ there are four zeros, or $N_{Z}=4$. Intermediate limits $(\times)$ are trivial solutions.

For proper convergence, many modes must be included in the cavity region, including Bessel functions of orders close to or greater than 100. However, with the arguments for the $P$-fields at radius $R_{c}$, the values of $J_{m}(\rho)$ become very small, while they become very large for $Y_{m}(\rho)$. This would result in a poorly-conditioned matrix. To keep the magnitude of the values at similar orders, and also not do computations with numbers that are near the limit of floating-point precision, an additional normalization is included with the $P$-modal coefficients. In effect, the Bessel functions and their derivatives are divided by the function values at the discontinuity radii.

Still, it was found with MATLAB that with orders of $m$ greater than about 100 , erroneous results of either 0 or inf are given although the function was still calculable in another mathematics software package Mathematica ${ }^{\circledR}$. To deal with this problem, the logarithm of the Bessel function was calculated with continued fractions as in 68 . Thus, when the normalization above is taken, the two more well-numerically behaved numbers can instead be subtracted.

\subsubsection{Aperture approximation}

At the $\rho=R_{c}$ boundary, the aperture fields must undergo a coordinate transformation so that the surface integrals can be expressed analytically in a common coordinate system. However, unlike the analysis of the discontinuity found in 2.2.1. the aperture and circular regions do not share a common normal vector across the whole surface area. This necessitates a numerical integration 
that includes the propagation constants, which are frequency-dependent, when otherwise the surface integrals are only dependent on the dimensions; this impacts the computation speed. For relatively large apertures the numerical integrals are necessary, but a small-angle approximation can be used when the aperture width is much smaller than the cavity circumference [59 60].

\subsubsection{Convergence}

The modeling will be exact if an infinite number of modes can be used, but since this is not possible, an upper limit needs to be set. Looking at the approximate fields in 2.2a and 2.2b), there is good consensus in the literature that a proper ratio between $L$ and $M$ needs to be chosen for accurate convergence $57,59,69,70$. For the analysis of the discontinuity between rectangular waveguide and bifurcated waveguide, Mittra and Lee found that the ratio of the number of modes in each subdivided portion of the bifurcated guide should be set equal to the ratio of their dimensions; the number of modes in the rectangular guide equals the sum of all of the modes in the bifurcated guide. This type of discontinuity can be turned into a step discontinuity by shorting one portion of the bifurcated guide, or setting its width as zero. It can then easily be found that the ratio of the number of modes of the two guides equals the ratio of their dimensions. This method has been adapted for twodimensional discontinuities 58,71 . Other authors set a cut-off frequency greater than the highest band frequency and include all modes that propagate below this frequency [59,60,70].

\subsection{Conclusion}

The electromagnetic theory of a circular cavity was introduced, along with its use in permittivity measurements. Full-wave modeling allows for more complex configurations of the circular cavity. An introduction to mode-matching was presented, and an overview of its application to modeling the permittivity setup was given. 


\section{Chapter 3}

\section{Liquid Measurement}

\subsection{Setup design}

Because rigorous modeling is used, which enables greater flexibility with permittivity characterization, the cavity was designed for measurement of liquids with varying permittivities, and at multiple frequencies around $100 \mathrm{GHz}$. To hold the liquid, fused quartz tubes were chosen, since they are low-loss and so maintain the high quality factors.

In addition, temperature and humidity controls were included in the design, serving to improve the data precision but also enhance the modeling accuracy - the waveguide dimensions and liquid properties are dependent on the temperature, and the permittivity of air is dependent on the relative humidity.

\subsubsection{Electromagnetic design}

As with nearly all waveguide components at higher millimeter-wave frequencies, the circular cavity is contained within a machined block. The negative space of this block, which is shown in Figure 3.1 . will define the cavity, the insertion hole for the tube, and the excitation feeds.

The blocks were machined out of aluminum and then plated with a layer of gold, which has a higher conductivity and does not oxidize. 


\section{Overall cavity configuration}

To simplify the modeling, the placement of the insertion hole for the tube is configured coaxially with the cavity. As a result, the coupling into the cavity is placed on the sidewall, perpendicularly to the tube. This is easier to configure than placing both the coupling and the tube insertion hole in the same plane, since the cavity block ultimately must be connected to flanges on the frequency multiplier heads of the network analyzer.

The output of the heads is rectangular waveguide, which sets the input and output feeds of the cavity. In order to machine these waveguide feeds with high precision and accuracy, the block must be split into two halves, cutting through the waveguide. There are two ways to split the waveguide, either through the longer wall (H-plane) or the shorter wall (E-plane). Since currents travel across the E-plane, parallel to the waveguide $\tilde{\mathbf{E}}$-field, they would be disturbed by a split placed in this wall, which would degrade the performance of the waveguide and add loss. Thus, the split was placed through the H-plane. Yet because a circular cavity is being used, this H-plane versus E-plane choice also determines which type of modes are excited, since the axis of the hollow right circular cylinder is more simply machined perpendicularly to the plane of the split. As a result, the orientation of the fields of the $\mathrm{TE}_{10}$ mode with respect to the cavity will couple to $\mathrm{TE}_{z}$ modes, which have magnetic fields in the $\hat{z}$-direction. Finally, the waveguide flanges of the frequency multiplier heads are actually oriented perpendicularly to the waveguide in Figure 3.1. So for measurement, this negative space is rotated $90^{\circ}$, and the tube lies horizontally.

\section{Dimensions}

A number of factors determined the block dimensions, which are summarized in Table 3.1, but they were largely based on a previous block that was acquired for an unrelated project of a millimeterwave magnetron that required a whispering gallery mode. With this block, liquid measurements were performed, giving data that guided the design of a new block. The major difference is that the previous block had TM mode-excitation, i.e. $90^{\circ}$-rotated rectangular waveguide, but based on simulations and closed cavity theory, the sensitivity was expected to be similar.

The major dimensions of the radius and height were kept the same. Closed-form equations for $f_{0}$ and $Q$ were used to verify that TE-modes of different electric field patterns, i.e. permittivity 


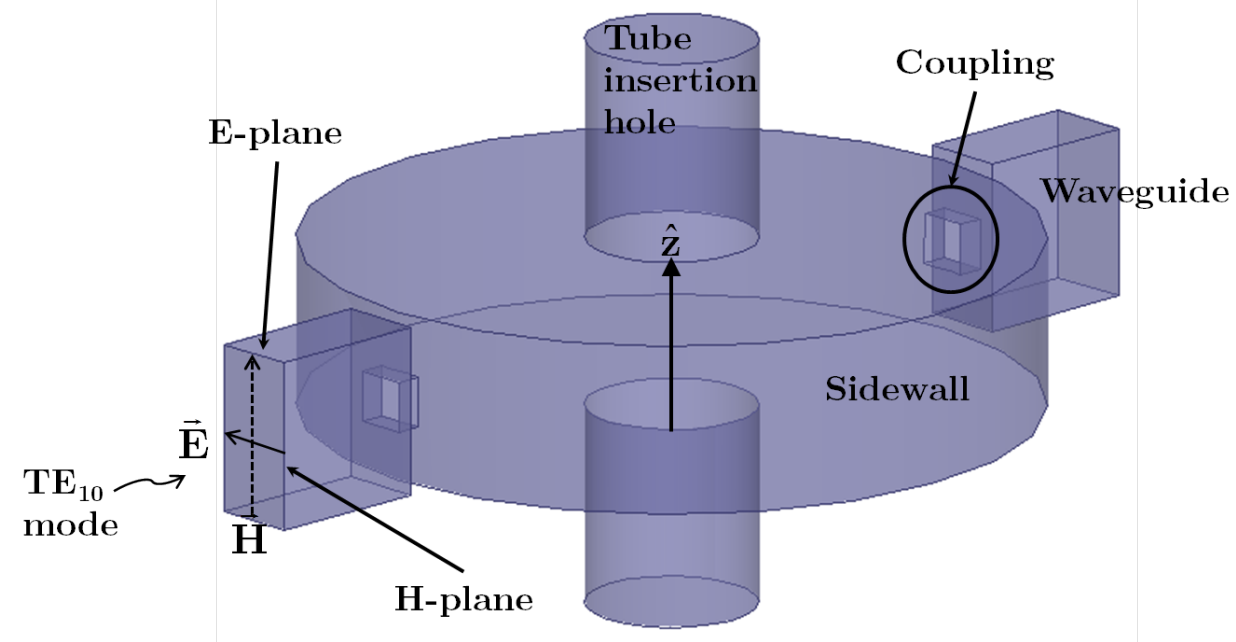

Figure 3.1: Diagram of negative space in a machined cavity block.

sensitivities, would be available in W-band; some of these field patterns can be seen in Figure 3.2 . Initial modeling, which included coupling to the cavity, verified this approach by matching the measured resonant frequencies of the old block to those of a closed cavity, based on the plotted electric fields.

The aperture size of the previous block was thought to be suitable. Maximum transmission $S_{21}$ values of the resonances ranged from $-15 \mathrm{~dB}$ to $-20 \mathrm{~dB}$, which is higher than normal for resonance measurements, but these systems typically measured low-loss materials at lower frequencies. With the available network analyzer in $\mathrm{W}$-band, the specified dynamic range is $76 \mathrm{~dB} 72$. However, additional waveguide components used for environmental control would reduce this dynamic range, and it was difficult to quantitatively anticipate the reduction. Additionally, the loss of the liquids would reduce the transmission level, particularly for sensitive modes with strong electric fields in the center.

The final dimensions that were considered were those regarding the tube. Obviously, the insertion hole must be made big enough to accommodate the maximum tube diameter. However, it cannot be made too big because the more sensitive modes will radiate away through the hole and no longer resonate. This can be ascertained by computing the cut-off frequencies of the lowest-order circular waveguide modes. With the previous block, MHz-shifts were seen for lossy liquids in a tube of a $1 \mathrm{~mm}$ outer diameter, so the inlet hole was made a bit larger than this. For this diameter, the 


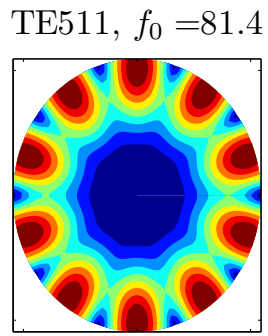

TE221, $f_{0}=83.2$

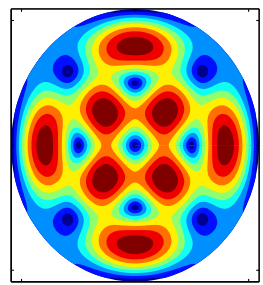

TE021, $f_{0}=85.1$

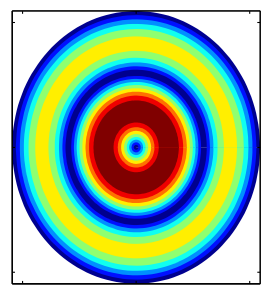

TE611, $f_{0}=88.2$

TE321, $f_{0}=91.6$

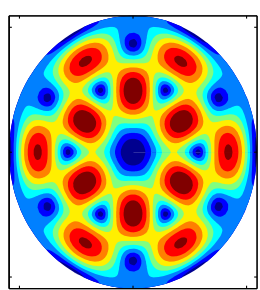

TE711, $f_{0}=95.4$

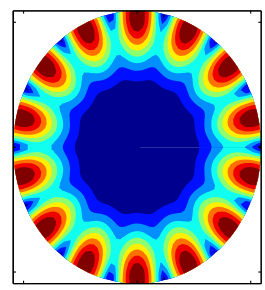

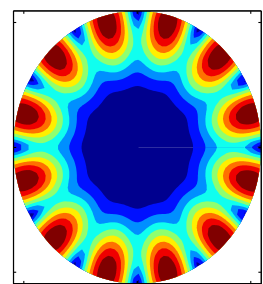

TE421, $f_{0}=100$

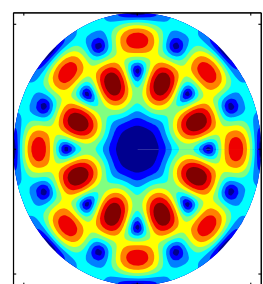

Figure 3.2: Electric field plots of TE modes $\left(f_{0}\right.$ in $\left.\mathrm{GHz}\right)$ in W-band.

Table 3.1: Nominal cavity dimensions

\begin{tabular}{|c|c|}
\hline Cavity radius & $5.461 \mathrm{~mm}$ \\
Cavity height & $2.540 \mathrm{~mm}$ \\
Hole radius & $1.066 \mathrm{~mm}$ \\
Aperture width & $762 \mu \mathrm{m}$ \\
Aperture height & $762 \mu \mathrm{m}$ \\
Aperture length & $381 \mu \mathrm{m}$ \\
\hline
\end{tabular}

corresponding cut-off frequency for the lowest-order mode $\mathrm{TE}_{11}$ is $175 \mathrm{GHz}$.

\subsubsection{Block exterior}

A number of features were added to the outside of the block, as seen in Figures 3.3 and 3.4 . The first two, (A1) and (B1), ensure that the tube is centered within the cavity, which is an assumption of the modeling. At the top of the block, the diameter of the (A1) hole was made to tightly fit a fluidic fitting that is placed over the tubes in order to facilitate the addition of liquid sample, as shown in the left in Figure 3.5. Otherwise, given the horizontal orientation of the tube, it would rest on the bottom half of the circumference of the insertion hole and not be centered. With the (A1) hole, the tube is centered to within $0.25 \mathrm{~mm}$. The bottom of the block was modified to accept an 
additional machined fitting (B1), with a smaller diameter hole in the center; it has alignment pins and screws on to the block.

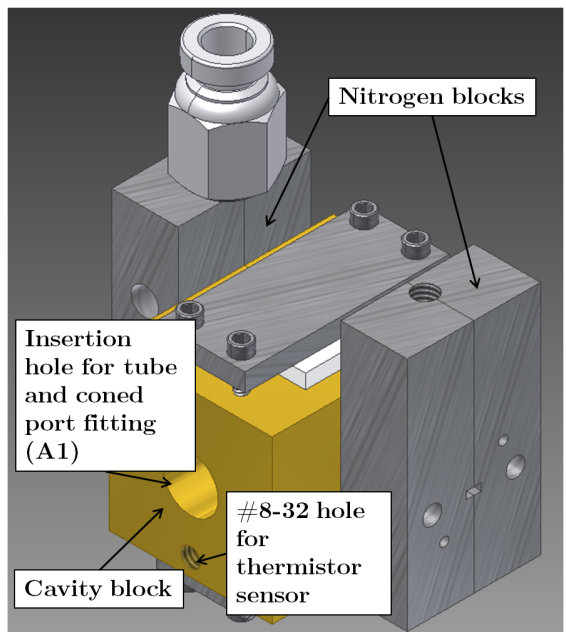

Figure 3.3: CAD image of block exterior, $0^{\circ}$ rotation.

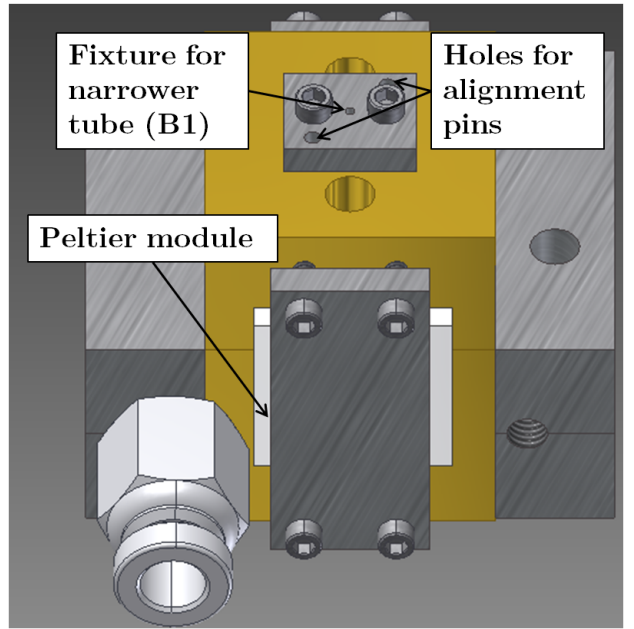

Figure 3.4: CAD image of block exterior, $90^{\circ}$ rotation.

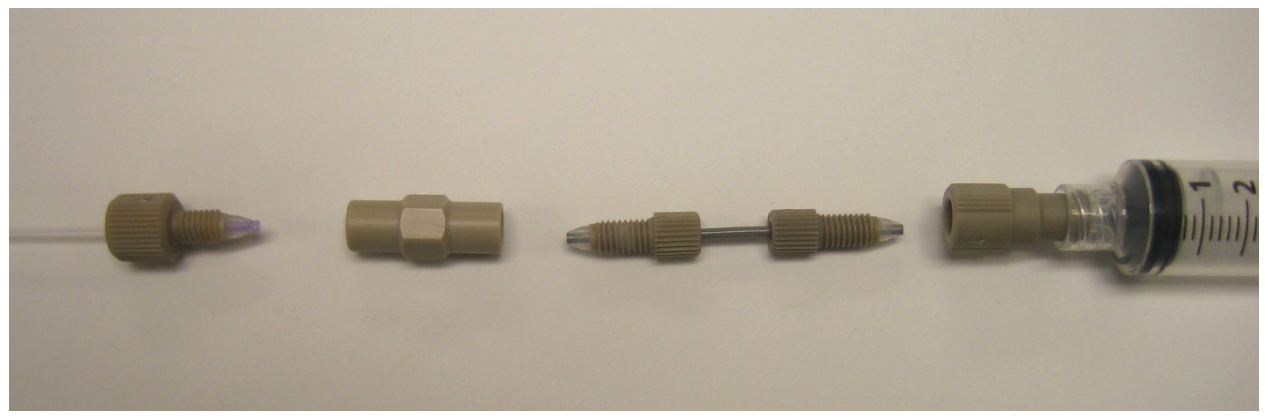

Figure 3.5: Fitting on quartz tube and connection to syringe.

\section{Humidity and vapor control}

On both sides of the cavity block are the Nitrogen blocks, which are shown in more detail in Figures 3.6 and 3.7. They consist of waveguide through sections with an inlet hole for a nitrogen gas feed that purges the cavity of water vapor or any vapor from the sample. In Figure 3.6. on the same face as the inlet hole are four holes which serve to fasten and align the two halves of a single Nitrogen block. To determine the diameter of the inlet hole and ensure it does not disturb 
power transmission through the waveguide, various values were simulated using the ANSYS®HFSS1 model shown in Figure 3.8. Ultimately the diameter of 0.04in was chosen, since the return loss is mostly above $20 \mathrm{~dB}$ across the entire band, and this larger diameter would require less pressure to purge the cavity. In order to prevent nitrogen from going into the extension heads and ensure flow through the cavity, the waveguide is sealed with a viscous epoxy.

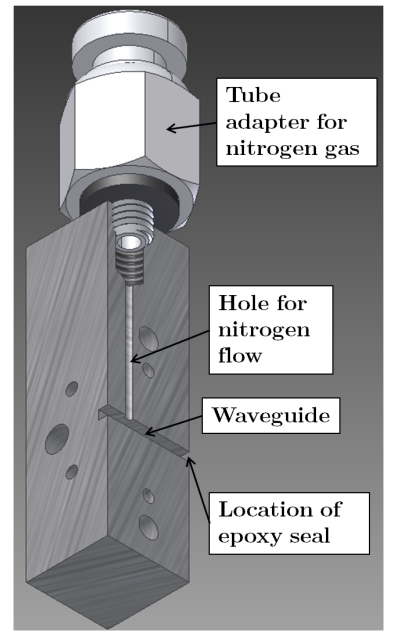

Figure 3.6: CAD image of Nitrogen block half ${ }^{a}$

${ }^{a} 3 \mathrm{D}$ model of tube fitting from McMaster-Carr Supply Company.

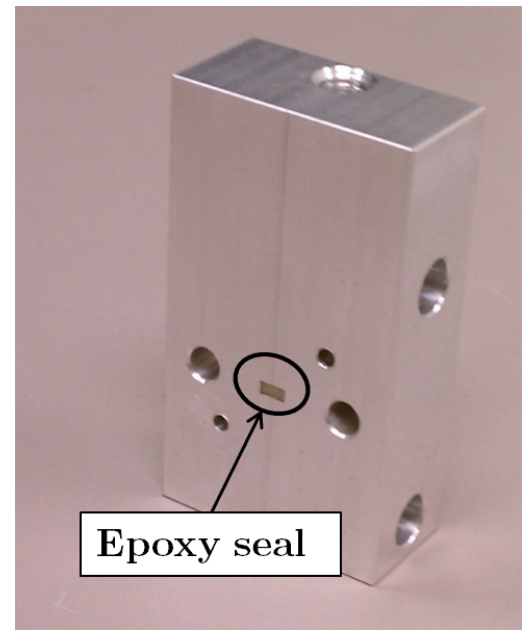

Figure 3.7: Photograph of Nitrogen block and epoxy seal.

\section{Temperature control}

Additional screw threads were added to the cavity block for temperature control. At the top of the block, the \#8-32 thread hole accepts a thermistor that is housed in a bolt mount with a \#8-32 thread. A thermistor was chosen because of its high sensitivity to small changes in temperature; the specific thermistor was the Omega ON-950 series thermistor. On each side of the block, screw threads were added in order to hold rectangular fixtures that can clamp Peltier elements or heaters onto the cavity block rather than gluing them, since it was desired to be able to separate the cavity block halves for cleaning. Ultimately Peltier elements, ones specifically from Laird Technologies, were chosen in order to have the flexibility for cooling the block.

\footnotetext{
${ }^{1}$ ANSYS, Inc., Canonsburg, PA
} 


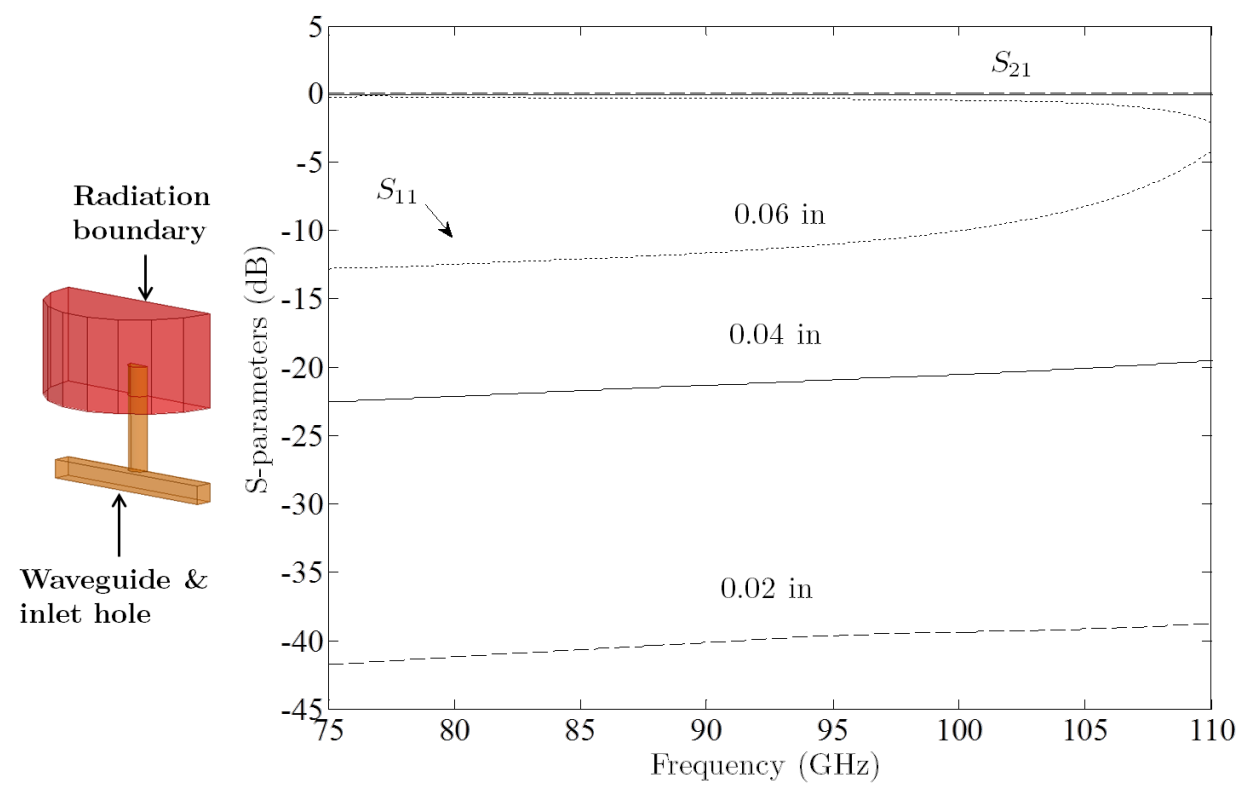

Figure 3.8: HFSS symmetrical model and simulated return loss of waveguide with inlet hole of varying diameters.

The temperature was controlled with an in-house PID program in Python, using PyVISA $2^{2}$ for GPIB control of the test equipment. To read the temperature, the thermistor leads were connected to an Agilent 34401 multimeter. In order to prevent self-heating of the thermistor, which would introduce error in the resistance readings, the resistance range was manually set to $100 \mathrm{k} \Omega$ so that only a $10 \mu \mathrm{A}$ current source is used, which is the recommended operating current for the thermistor. Resistance values were converted into temperature using the manufacturer-provided Steinhart-Equation for the thermistor. As for the Peltier elements, they were powered in parallel by an Agilent E3631A power supply.

\subsection{Measurement}

Achieving accurate measurements to compare to the modeling is dependent on many factors. First, the relative humidity of the air will affect its permittivity and thus alter the resonant frequency and quality factor. This is controlled by the Nitrogen blocks. Likewise any impurities, such as oils, on the surface of the cavity walls will have an effect. Next, because the Nitrogen blocks are

\footnotetext{
${ }^{2}$ T. Bronger et al., The PyVISA package, http://pyvisa.sourceforge.net/
} 
imprecisely plugged with a dielectric material, they cannot be accurately modeled and so must be included in the network analyzer calibration, which is a standard procedure that removes any phase and transmission errors in the system. Calibration also allows for the removal of the effect of any additional waveguide components necessary for measurement so that the data is only representative of the device under test. Finally, waveguide measurements in general are sensitive to connections made between components, such as a waveguide block to a flange. Both parts are machined with alignment pins and holes so as to reduce any reflections from offset waveguide cross-sections. This error becomes worse at higher frequencies due to the increased sensitivity of smaller wavelengths to machining tolerances. While the mode-matching technique can model offset waveguides, this is a random error that cannot be quantified accurately for each measurement. Two waveguides must also be in good physical contact with each other in order to maintain a consistent electric field boundary. So, waveguide flanges also include screw holes for tight fastening. This introduces another source of error, in that the measurement is sensitive to the torque of the screw.

All of these issues have either been addressed with the block design or will now be discussed in the measurement procedure, which is as follows. First, the VNA was powered on and left to warm up for at least one hour. Before data collection, the cavity block was cleaned of potential water vapor on the walls by heating it for at least thirty minutes, with a continuous Nitrogen flow; this was occasionally preceded by isopropanol clean of the cavity block in order to get rid of oils or other contaminants. Meanwhile, the Nitrogen blocks are placed on the extender heads so that their temperature is in equilibrium with the VNA before calibration. Next, a frequency range capable of capturing all resonance measurements is chosen, with the maximum number of swept frequencies and 128-pt averaging, which effectively helps to increase the dynamic range. A standard ThruReflect-Line (TRL) calibration is performed, which measures the known standards of a zero-length through waveguide, a $90^{\circ}$ delay line, and zero-length short.

Once the cavity has reached room temperature, it is connected in between the Nitrogen blocks, which is shown in Figure 3.9. To help with the temperature precision, styrofoam insulation is placed around the cavity block, as seen in Figures 3.10 and 3.11 in the front of the setup, the two insulation layers are tiled to allow for easy and fast access to the tube. The temperature of the cavity is monitored, and once it stabilizes, PID control is started. Data is collected once the control 
temperature is reached and stabilized.

For consistency and the reduction of measurement error, calibration standards and the blocks are fastened to the flanges using a constant torque wrench set at 4 in-lb. There is a noticeable effect on the transmission magnitude due to changes in torque with inconsistent hand-tightening; this would also change the coupling to the cavity, which effects the resonant frequency and quality factor.

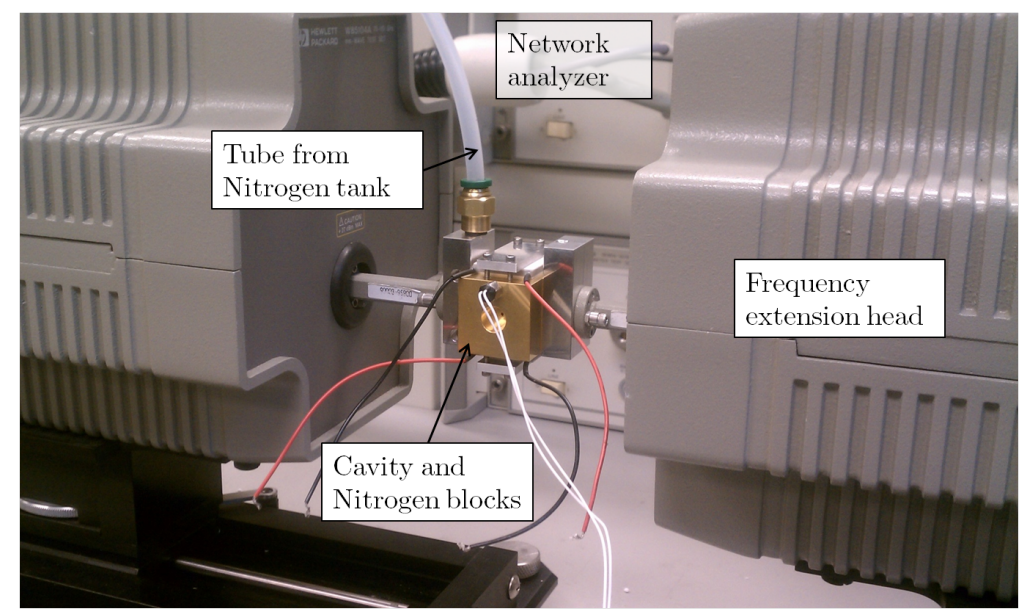

Figure 3.9: Cavity and Nitrogen blocks (with Nitrogen feed tube) connected to frequency extension heads, which connect to the network analyzer. Peltier elements and thermistor are installed onto cavity.

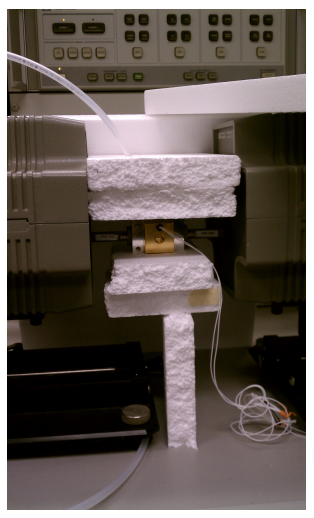

Figure 3.10: Inner view of insulation.

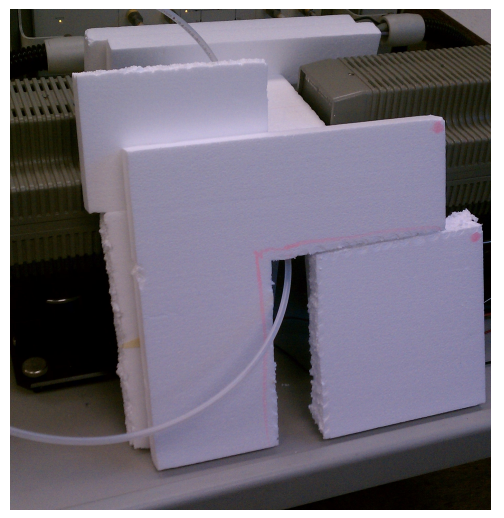

Figure 3.11: All insulation for measurement, with second nitrogen gas tube for quartz tube.

For the measurement of the empty tube, the nitrogen connection is split so that half of the gas flows through the tube. From Figure 3.5, the fitting over the tube has a \#10-32 thread, so it can 
be connected to the same tube fitting used for the Nitrogen block, via a female standoff. Finally, measurement of a liquid can be seen in Figure 3.12 .

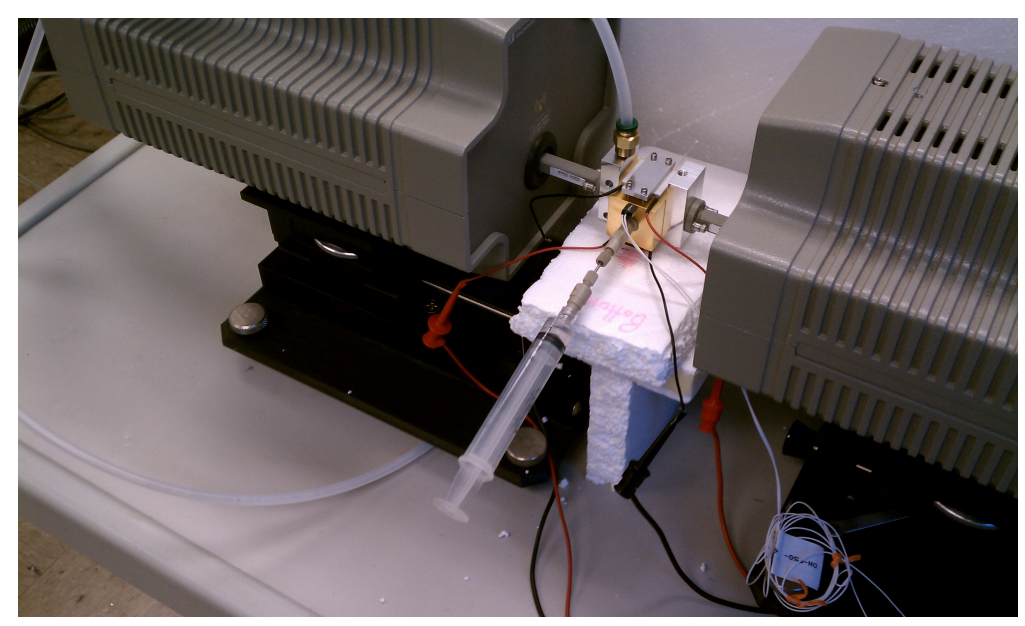

Figure 3.12: Injection of liquid sample into tube.

\subsection{Data post-processing}

Commonly $f_{0}$ can be found from the frequency of maximum $S_{21}$; subsequently $Q$ is found from $f_{0}$ divided by the difference between the $3-\mathrm{dB}$ frequency points, i.e. the frequencies at which the magnitude drops three decibels from the maximum value. However, this method is not very accurate when there is noise in the measurement. In a study comparing various resonance fitting procedures, Petersan and Anlage found that the phase versus frequency fit was the most accurate and precise 73 . For this method, a circle is first fit to the pairs of real and imaginary $S_{21}$ data; if there is no crosstalk nor phase shift then this circle should lie on the $x$-axis 74 . The circle fit gives information on the location of the $S_{21}$ circle in the complex plane. Then, the data is rotated and translated so that the center of the circle is placed at the origin. The phase of this shifted data is then used for fitting of this equation

$$
\phi(f)=\phi_{0}+2 \arctan \left[2 Q\left(1-\frac{f}{f_{0}}\right)\right]
$$

where $\phi_{0}$ is the angle where $f_{0}$ lies. Material and dimensional parameters were estimated from $f_{0}$ and $Q$ instead of to the sweeps directly because it was found to converge faster and more accurately. 
If a fit is to be made to a sweep, then it has to be assumed that the modeling is exactly comparable to the measurement, which is only the case if the system is perfectly calibrated. However, the calibration is not done at the same temperature as the measurement, so it will no longer be as accurate when the waveguide sections in the Nitrogen blocks expand with temperature. The phase versus frequency fit accounts for this and other systematic differences 7375 . 


\section{Chapter 4}

\section{Results}

In this chapter, the data acquisition process is broken down in parts to determine the uncertainties added at each step. The enviromental controls are assessed, and permittivity results are analyzed.

\subsection{Resonance fitting}

An example of resonance measurement and its circle fit is shown in Figure 4.1. Based on the center of the circle fit, the data is rotated and translated until its center lies at the origin. The phase of this data and the fit is shown in Figure 4.2. For the estimated parameters $f_{0}$ and $Q$ in vector $a$, the uncertainties $\sigma_{a}^{2}$ are found from the diagonal values of the covariance matrix, which is the inverse of the following matrix $\alpha$ 76

$$
\alpha_{j k}=\sum_{i}^{N_{i}} \frac{1}{\sigma_{i}^{2}} \frac{\partial y \prime\left(x_{i}, a\right)}{\partial a_{j}} \frac{\partial y \prime\left(x_{i}, a\right)}{\partial a_{k}}
$$

where the uncertainties $\sigma_{i}$ are equivalent and estimated from the quality of the fit

$$
\sigma^{2}=\frac{1}{N_{i}-N_{a}} \sum_{i}^{N_{i}}\left[y_{i}-y\left(x_{i}, a\right)\right]^{2}
$$

and $N_{i}$ is the number of data points, $N_{a}$ is the number of parameters, $x_{i}$ are the independent data points, $y_{i}$ are the measured data points, and $y\left(x_{i}, a\right)$ is the best fit from a nonlinear-least squares 
method. For the resonance in Figure 4.2, values and uncertainties found from the fit are summarized in Table 4.1 .

Table 4.1: Phase fit example values

\begin{tabular}{|c|c|c|}
\hline & $\mathrm{a}$ & $\sigma_{a}$ \\
\hline$\phi_{0}$ & -0.092 & 0.00046 \\
$f_{0}$ & $83.026360 \mathrm{GHz}$ & $9 \mathrm{kHz}$ \\
$Q$ & 6525 & 9 \\
\hline
\end{tabular}

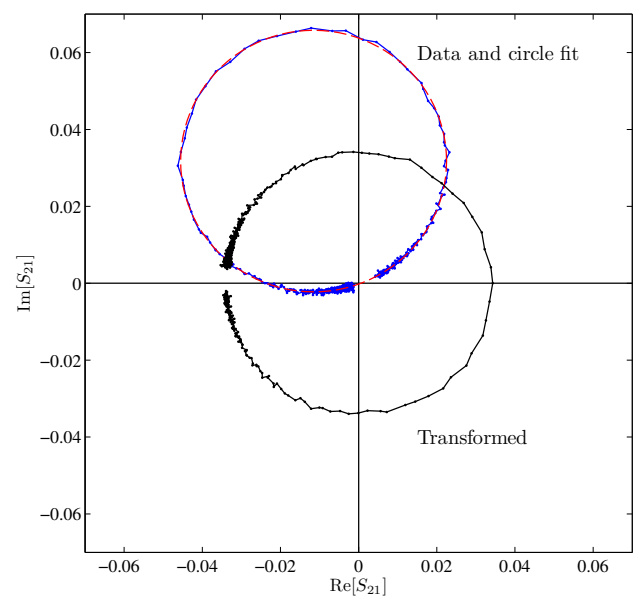

Figure 4.1: Example of circle fit (red) to original complex $S_{21}$ data (blue) and its transformation (black).

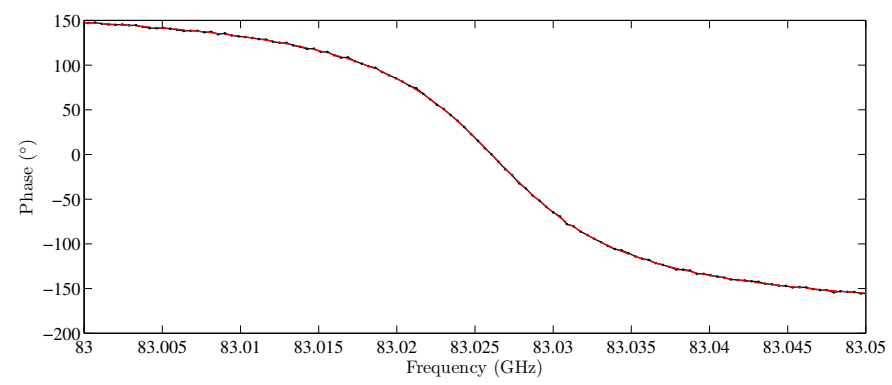

Figure 4.2: Example of phase fit (red) to phase of transformed $S_{21}$ data (black). 


\subsection{Measurement setup}

The performance of the environmental controls are discussed. With repeated measurements, uncertainties in the data were characterized so that further on the precision of the permittivity results can be found. Variability in measurements within a single day and across multiple days were examined, along with the possible effects of the environmental controls and mechanical connections.

\subsubsection{Environmental controls}

After the Nitrogen blocks were sealed with epoxy, they were measured on the network analyzer. The insertion loss is mostly less than $5 \mathrm{~dB}$, while the return loss is mostly above this value; the exception is at the lower part of the band. Also, the reflection $S$-parameters of $S_{11}$ and $S_{22}$ are different from one another due to the unequal amounts of epoxy in each block. The high loss due to the epoxy lowered the available dynamic range of the system when it was calibrated with the Nitrogen blocks at both ports. An example calibrated measurement is shown in Figure 4.4 .

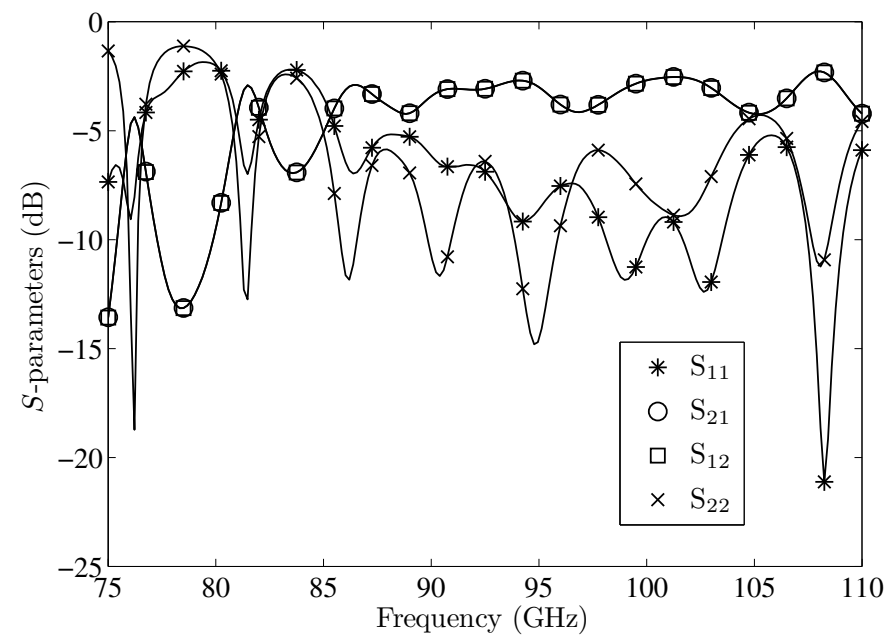

Figure 4.3: Measured $S$-parameters of Nitrogen blocks. The high loss is due to the epoxy.

With the humidity control there was concern over the affect on the measurements from the nitrogen pressure level, which was set by the tank regulator with pressure markings larger than the actual pressure needed for purging. This was tested by increasing the pressure until it was slightly above the range of operator uncertainty, which was judged by the volume of the sound of the gas; 


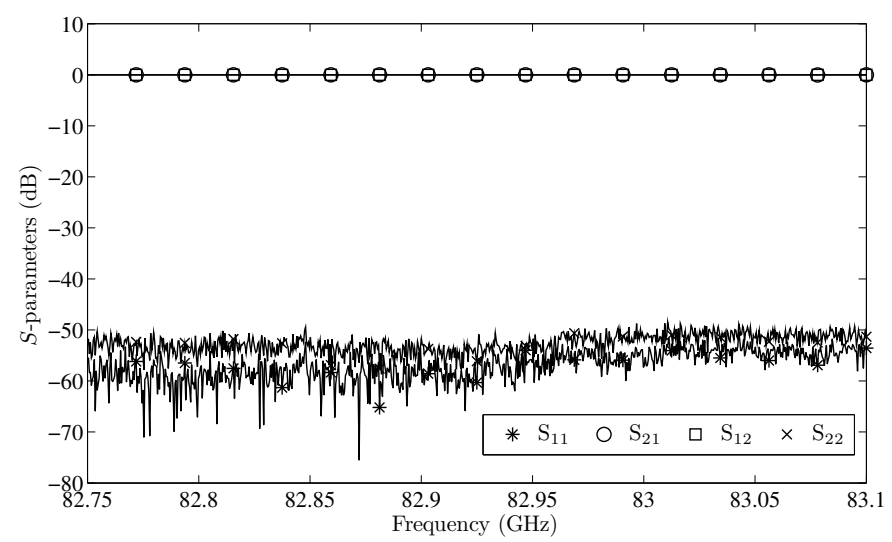

Figure 4.4: Measured $S$-parameters of the Thru section after TRL calibration with Nitrogen blocks.

the effect on $S_{21}$ is seen in Figure 4.5. There was a $200 \mathrm{kHz}$ decrease in the resonant frequency and no discernible effect on the quality factor.

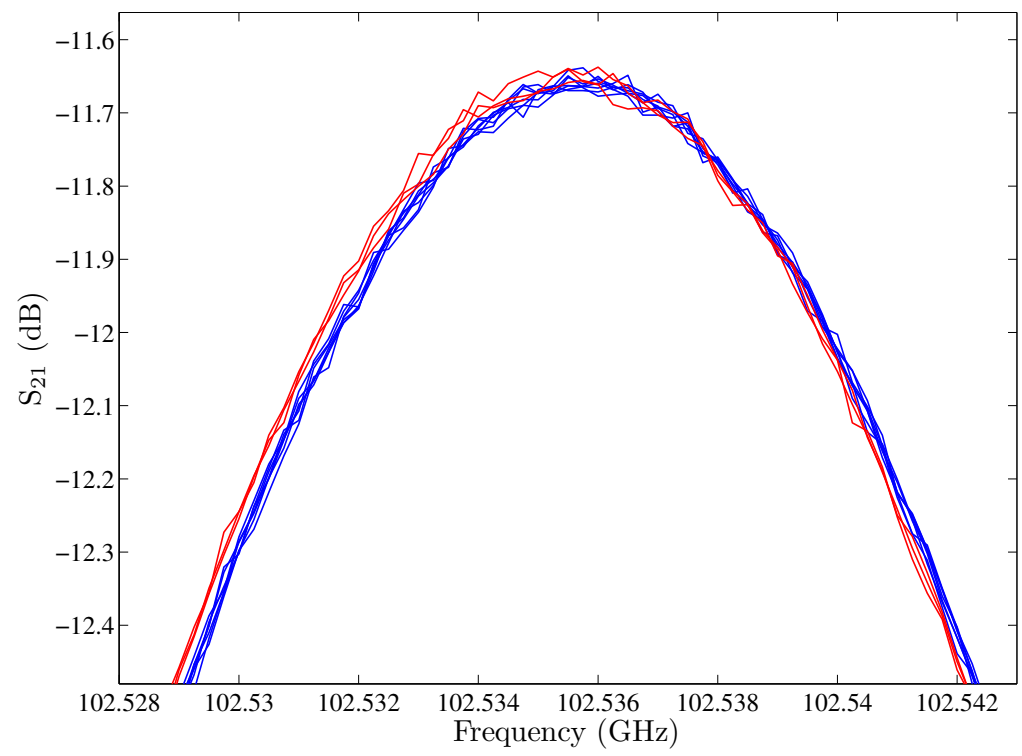

Figure 4.5: Effect of different $\mathrm{N}_{2}$ pressures. Change in resonance measurement. Red is increased pressure, with $f_{0}$ reduced by $200 \mathrm{kHz}$.

With the PID controller and insulation, the achievable temperature precision ranged from $\pm 0.01^{\circ} \mathrm{C}$ to $\pm 0.05^{\circ} \mathrm{C}$. Manual tuning was done to find the three PID constants. The Integral term was left at zero because when correcting for the below-setpoint cavity temperature during initial heating, the accumulated error prevented the block to cool back down to setpoint, especially with the insulation 
surrounding the cavity. A plot of typical temperature control is seen in Figure 4.6. At the moments in time where the temperature goes beyond this precision, either the PID control was initiated, or the empty tube was inserted into the cavity or filled with liquid, so the insulation was removed in order to obtain access to the cavity. Another example of the temperature control is seen in Figure 4.7, in which the temperature was held steady for close to an hour while the current adjusted for changing room temperature. Additionally, the plot also shows a decrease in setpoint by $1^{\circ} \mathrm{C}$, followed by an increase of $0.1^{\circ} \mathrm{C}$.

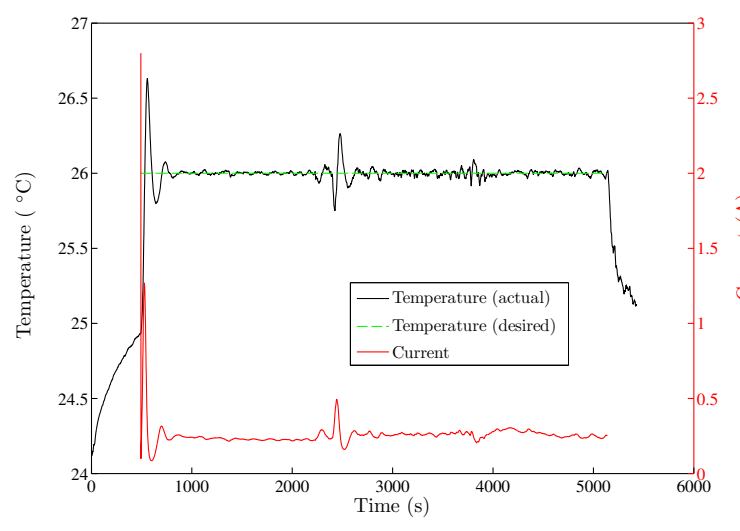

(a) PID control of temperature.

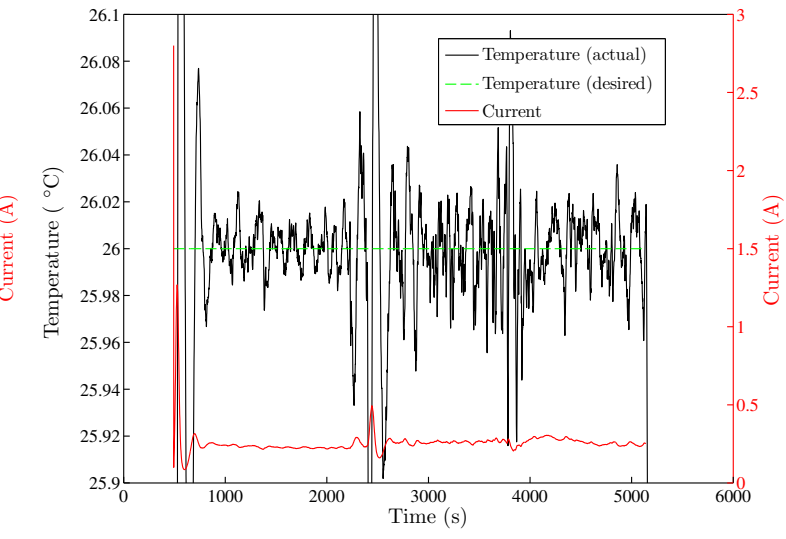

(b) Close-up of temperature precision.

Figure 4.6: Typical temperature control.

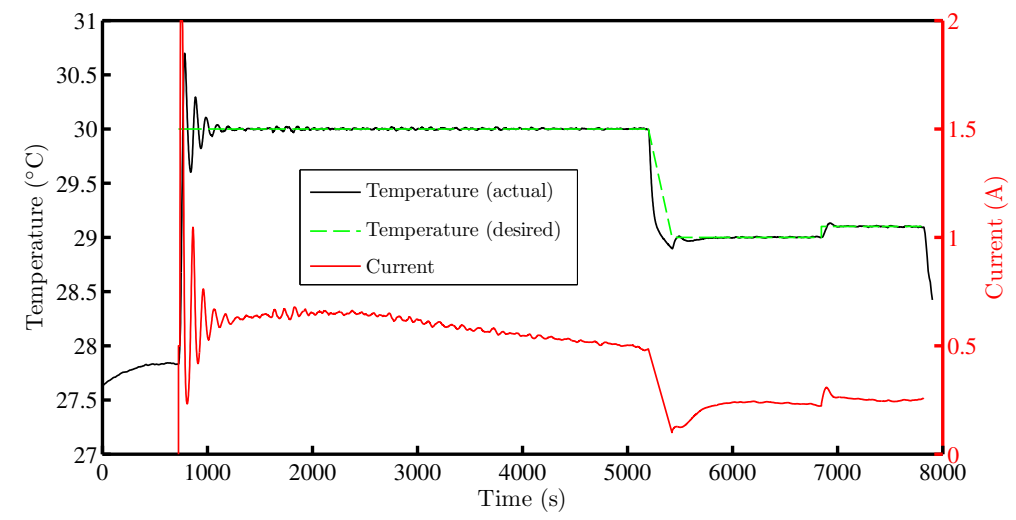

Figure 4.7: Example of stable temperature hold and multiple setpoints. 


\subsubsection{Data variability}

The sweeps in Figure 4.5 also show the typical variation in the measured resonances for a single day of measurements. Before liquid data was taken, a variety of empty cavity measurements were done to test the stability and repeatability of the setup. First, data was acquired over multiple days, using an identical Aluminum block with no gold plating, shown in Figure 4.8 The first day of data was near $102.462 \mathrm{GHz}$, and on the following day $f_{0}$ rose by about $2 \mathrm{MHz}$ and $Q$ by 30 . On the three final days, the changes seemed to have leveled off; the data is concentrated within a $3 \mathrm{MHz}$ range, and the $Q$ varies by no more than 10 . Means and standard uncertainties for each day are shown in Table 4.2. It should be noted that for days (2)-(5), the data was collected during a span of over one hour. Also in the table, the final means and uncertainties for all five days are shown in the final row. The standard uncertainty of parameter $a$ is a combined uncertainty and is defined as

$$
u_{a}^{2}=\frac{\sigma^{2}}{N}+u_{a^{\prime}}^{2}
$$

where $\sigma$ is the standard deviation and $N$ is the number of data points. The variable $u_{a^{\prime}}$ is the uncertainty of a data point - in a single day this comes from the phase fit, and over multiple days this is the uncertainty in a single day of measurements. The upward trend in the data indicated that perhaps the repeated heating and purging of the cavity was clearing it of impurities, so various cleaning steps were added and tested, which are summarized in Table 4.3 with the corresponding data shown in Figure 4.9. These measurements were done with the gold-plated cavity at $28^{\circ} \mathrm{C}$, which is why the quality factors and resonant frequencies are higher. First, an isopropyl alcohol (IPA) clean of the cavity was done to remove any oils on the inner block faces. Next, a heating step with the Peltier modules was added to try to remove any water vapor on the walls; during the heating, nitrogen flowed continuously through the cavity. From previous measurements, it was observed that in a single day $f_{0}$ was consistently precise to $1 \mathrm{MHz}$, so this was the targeted metric. Yet, since the spread in the data for the IPA clean-60 minutes heating $+\mathrm{N}_{2}$ flow- $\mathrm{N}_{2}$ flow sequence (denoted by symbol $\bullet$ ) was over $5 \mathrm{MHz}$, a final day of measurements was attempted without the heating step. After the block reached a stable temperature and the $20 \mathrm{MHz}$ decrease in $f_{0}$ was observed, it was heated for 30 minutes, re-measured, then re-heated for an additional half-hour. 
There was no significant change in $f_{0}$. If the 60 minutes heating et al. data are solely considered, then $u_{f 0}$ and $u_{Q}$ drop to $0.5 \mathrm{MHz}$ and 3.7, respectively. However, the final measurement, with very similar cleaning procedures, was more than $20 \mathrm{MHz}$ off of these measurements, so further tests were done to investigate possible causes of the drift that are independent of possible cavity impurities.

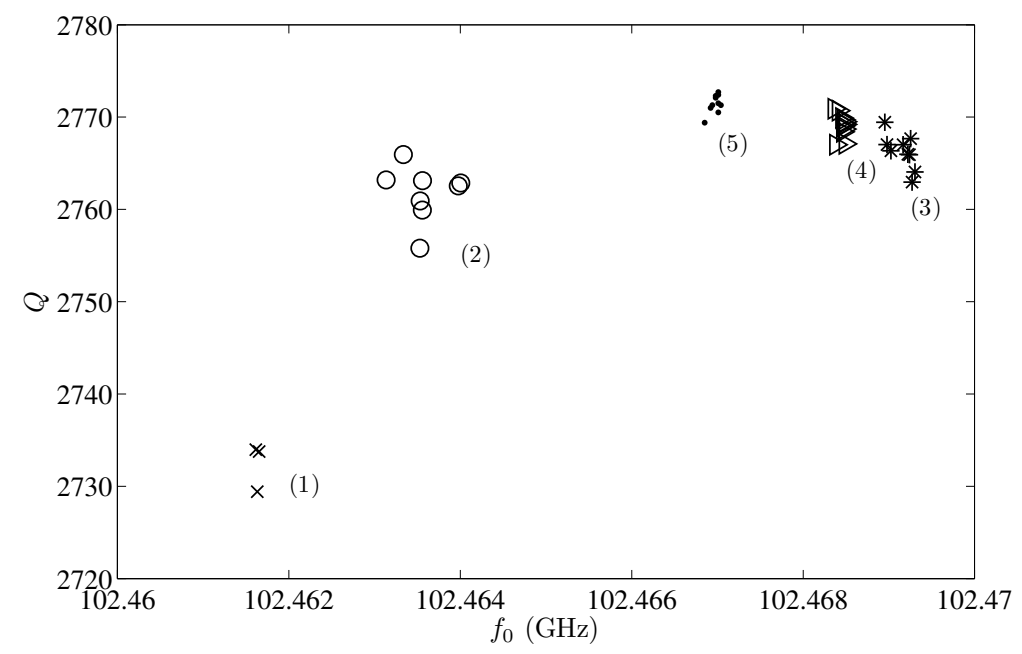

Figure 4.8: Variation of data for $\mathrm{TE}_{811}$ mode at $30^{\circ} \mathrm{C}$ over five days. Aluminum block measured.

Table 4.2: Al cavity, $\mathrm{T}=30^{\circ} \mathrm{C}$

\begin{tabular}{|r|c|c|c|c|}
\hline & $f_{0}(\mathrm{GHz})$ & $u_{f_{0}}(\mathrm{KHz})$ & $Q$ & $u_{Q}$ \\
\hline$(1)$ & 102.4616 & 18 & 2732 & 2.5 \\
$(2)$ & 102.4636 & 105 & 2762 & 2.3 \\
$(3)$ & 102.4692 & 46 & 2766 & 1.4 \\
$(4)$ & 102.4685 & 13 & 2769 & 0.6 \\
$(5)$ & 102.4670 & 17 & 2771 & 0.6 \\
\hline All days & 102.4660 & $1.4 \mathrm{MHz}$ & 2760 & 7.3 \\
\hline
\end{tabular}

Table 4.3: Au cavity $\mathrm{TE}_{811}$ cleaning steps

\begin{tabular}{|r|l|}
\hline Symbol & Cleaning procedures \\
\hline$\square$ & Isopropyl alcohol (IPA) clean-30' heating $+\mathrm{N}_{2}$ flow- $\mathrm{N}_{2}$ flow \\
$\circ$ & $60^{\prime}$ heating $+\mathrm{N}_{2}$ flow- $\mathrm{N}_{2}$ flow \\
$\bullet$ & IPA clean- $60^{\prime}$ heating $+\mathrm{N}_{2}$ flow- $\mathrm{N}_{2}$ flow \\
$*$ & IPA clean-30' heating $+\mathrm{N}_{2}$ flow- $30^{\prime}$ heating $+\mathrm{N}_{2}$ flow- $\mathrm{N}_{2}$ flow \\
\hline
\end{tabular}




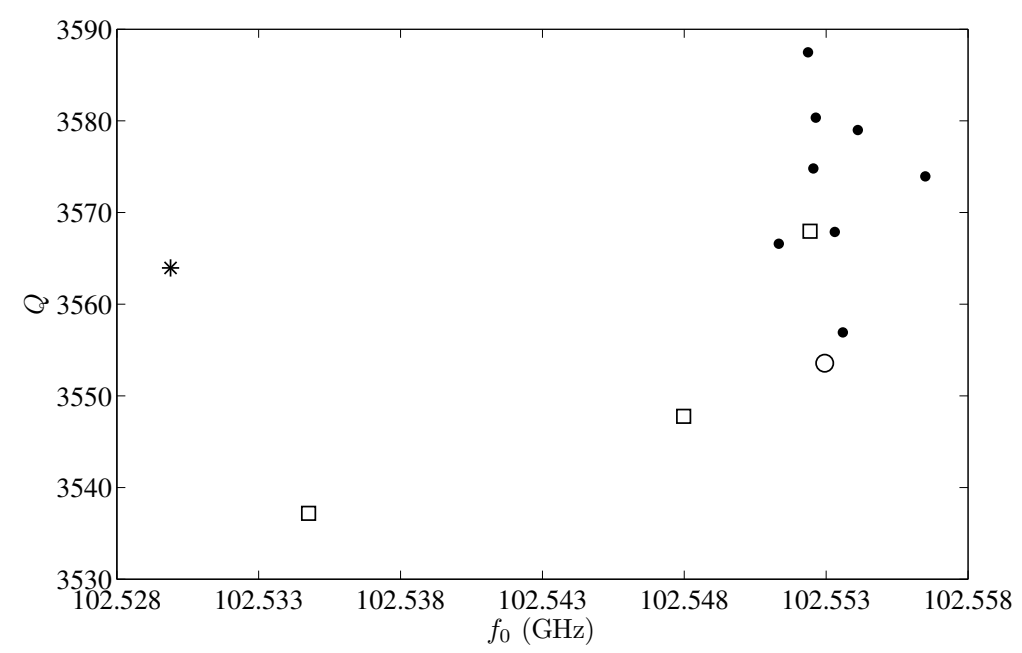

Figure 4.9: Variation of data for $\mathrm{TE}_{811}$ mode at $28^{\circ} \mathrm{C}$ over thirteen days. Au-plated block measured. Mean $f_{0}=102.5496 \mathrm{GHz}, Q=3565 . u_{f_{0}}=2.2 \mathrm{MHz}, u_{Q}=4.0$

\subsubsection{Repeatability tests}

Other causes for variability among day-to-day measurements come from variation in the assembly of the cavity block halves and connection of the block to the extension head flanges. For the block halves and flanges to align, the pins must obviously fit in the alignment holes, but with machining tolerances, there is room for error through which lateral and/or rotational misalignments can be introduced, on the order of a few microns. Also, variation in the torque when fastening the block halves together or the block to the flanges will have an effect as well. Namely, the shape of the cavity will be altered and also the coupling to the VNA waveguides. These effects were examined via measurements.

Two sets of raw data (i.e. not calibrated), with temperature control and nitrogen flow, were taken on consecutive days, while in between the cavity was left connected to the network analyzer; the measurements are shown in Figure 4.10. From the curve-fitting results, the resonant frequencies are indistinguishable, and the Q-factors differ by $0.1 \%$. The only major difference is the magnitude shift in $S_{21}$, but this is expected for uncalibrated data. These results indicate how well the $\mathrm{N}_{2}$ purge and temperature control aid in the repeatability of the data, although more consecutive days of measurements would be needed to state this with great certainty.

To test the effect of variability with assembly of the cavity block halves, the cavity was dis- and 


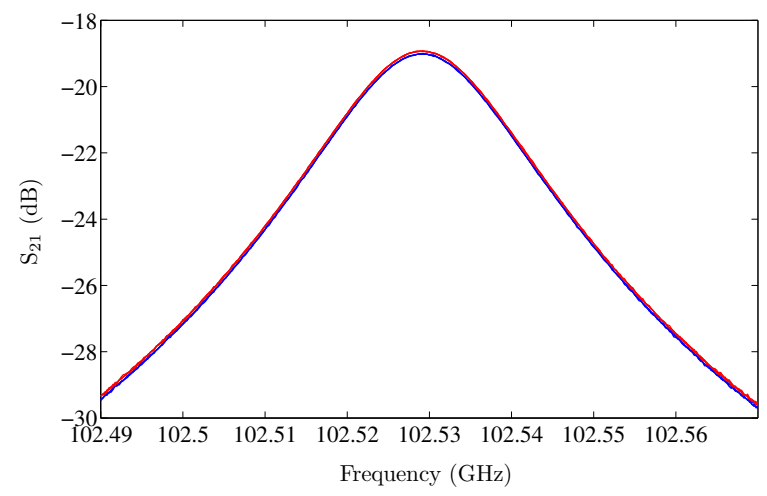

Figure 4.10: Raw data of empty cavity resonance, measured on two consecutive days, with cavity block left connected to VNA.

reassembled multiple times during a single-day measurement set. Once the temperature restabilized when the cavity was fastened back on to the VNA extension heads, data was recorded. This experiment was done for two different resonant modes, and the data can be seen in Figure 4.11 . It can be seen that the resonant frequency can vary within 5-6 MHz. Also examined was the effect of variability in the connection of the cavity block to the extension head flanges, as seen in Figure 4.12 Four times the cavity was disconnected and reattached, with the block halves kept fastened together; the resonant frequency varies within less than $1 \mathrm{MHz}$. In the same day, the re-assembly of the cavity was also tested and again a maximum shift of $5 \mathrm{MHz}$ was seen.

Because these measurements were taken in the same day and with the same cleaning procedure, any cavity impurities were presumably equivalent, so the larger shifts in the data are due to changes in assembly of the cavity block halves, which cannot be modeled. This observed $5 \mathrm{MHz}$ variation likely explains the variation of most of the data in Figure 4.9. The two outliers are then probably due to poor block assembly and/or flange connection.

From these experiments, estimates for the uncertainties in $f_{0}$ and $Q$ are found, which are later used in the uncertainty analysis for modeled parameters. For now, the variation in $f_{0}$ due to block disassembly is not included, but will be examined later. Remaining measurement uncertainties are listed in Table 4.4. The uncertainty due to the imprecise nitrogen pressure is treated as a Type B uncertainty, where the $200 \mathrm{kHz}$ seen in Figure 4.5 defines a half-width of a rectangular distribution; thus this value is divided by $\sqrt{3} 77,78$. Next, the effect of the flange connection is included by 


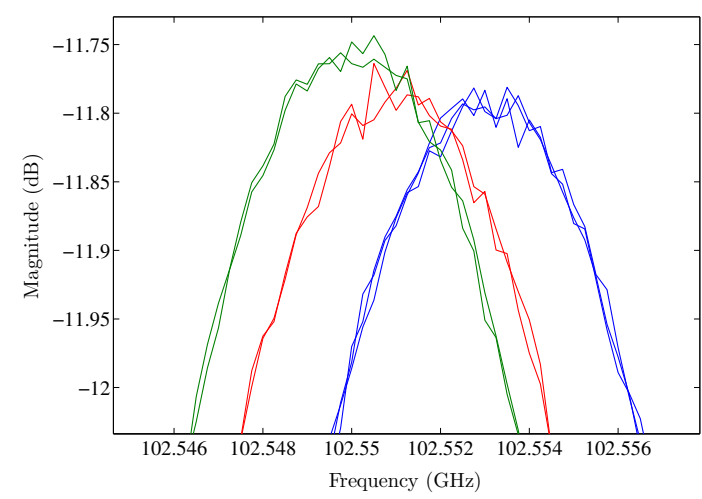

(a) $\mathrm{TE}_{811}$

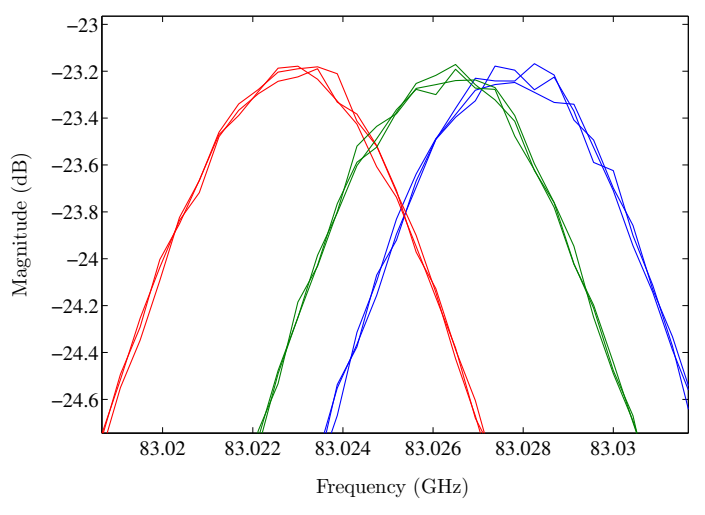

(b) $\mathrm{TE}_{221}$

Figure 4.11: Effect on resonances of dis- and reassembly of cavity.

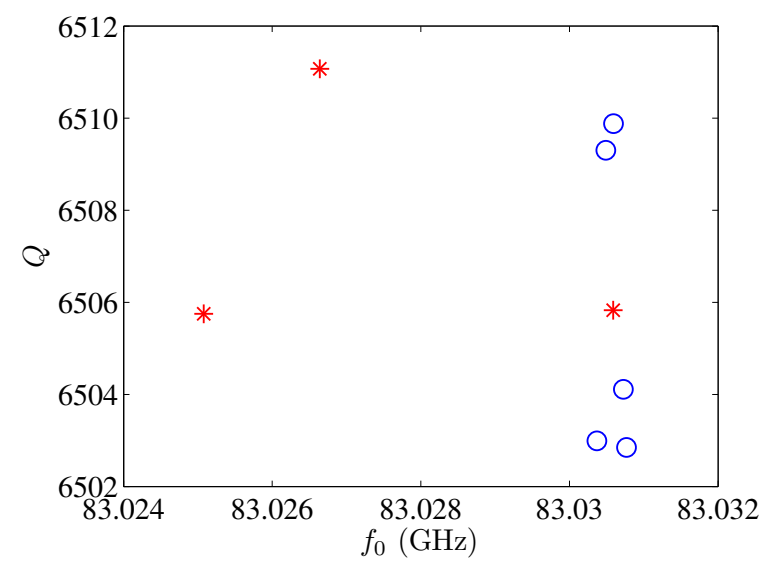

Figure 4.12: Effect of reconnection (०) and reassembly $(*)$ of cavity on data.

estimating the standard uncertainties of the measurements in Figure 4.12. Finally, the same is done with the final three measurements of Table 4.2 in order to reflect the day-to-day uncertainty that was consistently seen with other resonant modes, without disassembly.

Table 4.4: Uncertainties in $f_{0}$ and $Q$

\begin{tabular}{|r|c|c|}
\hline Uncertainty source & \multicolumn{2}{|c|}{ Standard uncertainty } \\
\cline { 2 - 3 } & $u_{f_{0}}(\mathrm{MHz})$ & $u_{Q}$ \\
\hline Nitrogen pressure & 0.12 & - \\
Flange connection & 0.08 & 5.7 \\
Day-to-day & 0.64 & 1.7 \\
\hline Combined & 0.66 & 5.9 \\
\hline
\end{tabular}




\subsection{Modeling}

Application of the full-wave modeling described previously will now be discussed. In order to extract permittivity from measurements, the modeling must accurately capture the effects due to aperture coupling, conductor loss, and complex permittivity. Also, since the modeling is an approximate technique, the convergence of results with additional modes is examined, along with possible errors due to the small-angle approximation used at the aperture-cavity boundary.

\subsubsection{Validation}

The modeling was validated by comparing its relative changes in $S_{21}$ of the empty cavity versus the tube-loaded cavity between the measurements. Nominal dimensions and material properties were used in the modeling (i.e. no attempt was made to modify the model parameters to account for variations in the physical cavity). First, a whole band comparison can be seen in Figure 4.13 , where the modeled tube perturbations follow those of the measurements, particularly with the larger shifts that correspond to modes with strong, centrally-located electric fields, such as $\mathrm{TE}_{221}$ near $83 \mathrm{GHz}$ $\mathrm{TE}_{021}$ near $85 \mathrm{GHz}$, and $\mathrm{TE}_{131}$ near $95 \mathrm{GHz}$. An additional comparison of the phase for the empty cavity is seen in Figure 4.14 .

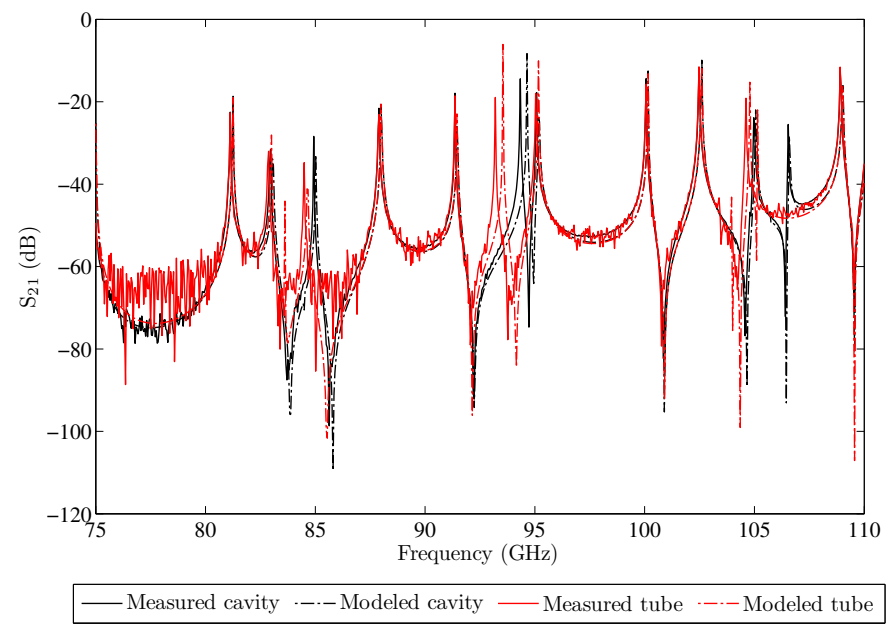

Figure 4.13: Measurement vs. modeling of empty cavity and tube, using nominal dimensions. 


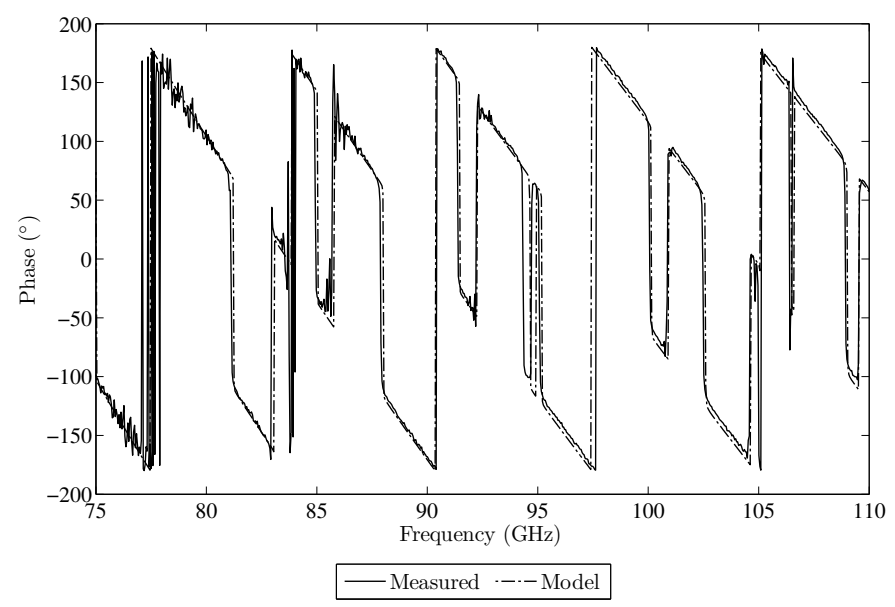

Figure 4.14: Measurement vs. modeling of empty cavity, phase, using nominal dimensions.

Additionally, with some resonances there is a behavior that could not be predicted with perturbation theory. For example, raw data measurements of the $\mathrm{TE}_{811}$ mode and its change with the tube and methanol is shown in Figure 4.15a. In spite of the additional loss of the quartz tube, the magnitude of $S_{21}$ increases. Furthermore with methanol, the resonant frequency decreases from that of the empty tube. These conditions were simulated, and the sweeps are seen in Figure 4.15b since the measured data was uncalibrated, the absolute magnitudes will not match up. Nominal values for the dimensions and materials are used, so while the resonant frequencies do not match up, the relative changes are being captured with the modeling.

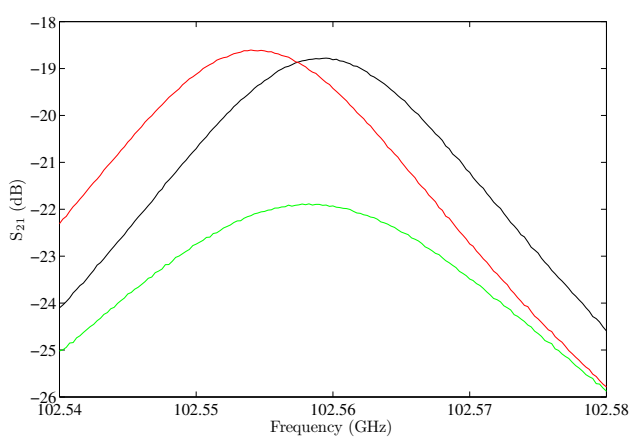

(a) Measured

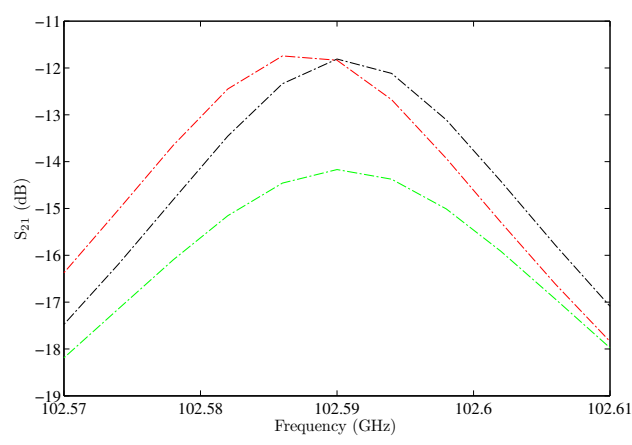

(b) Modeled

Figure 4.15: $\mathrm{TE}_{811}$ mode (black), with tube (red) and methanol (green). Comparison of measured versus modeled relative shifts. Measurements were raw, uncalibrated VNA data. Nominal cavity dimensions and permittivity literature values were used for modeling. 


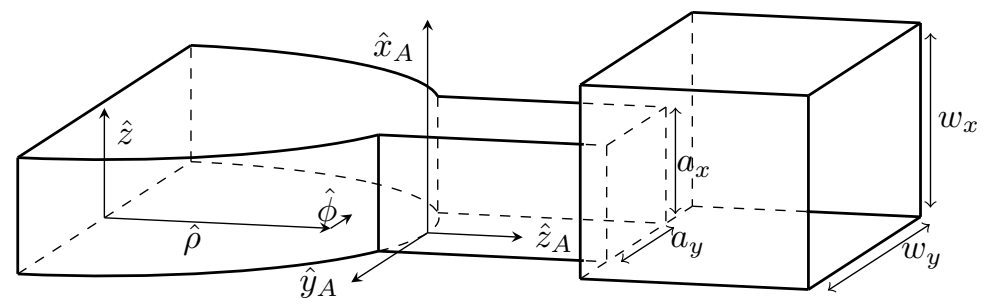

Figure 4.16: Diagram of structure to illustrate choice of modal limits by dimensional ratios.

\subsubsection{Convergence}

Since the permittivity fitting is done to the resonant frequency and quality factor, these terms were tested when examining convergence. A simple test of $S_{21}$ convergence for a single frequency could be tested, provided that the chosen frequency lies on a resonance. Convergence was examined by adding modes to the aperture region, or more specifically with different permutations of $M_{x}$ and $N_{y}$, which are respectively the maximum $m$ and $n$ indices of the aperture fields, as seen in A.26). In the waveguide region, the maximum index number is set by multiplying the maximum aperture index by the ratio of the corresponding parallel waveguide and aperture dimensions; for instance, in the $x_{A}$-dimension seen in Figure 4.16, $M_{x, w}=\frac{w_{x}}{a_{x}} M_{x}$. As for the cavity, the maximum $m$ for the regions within the cavity - A.4, A.16, and A.21 - is scaled from $N_{y}$ by the ratio of $\pi$ and the angle swept by the aperture arc. The index of $m$ is the variation of the fields in $\hat{\phi}$, which is mostly parallel to $\hat{y}_{A}$ along the aperture. In the $\hat{x}_{A}$ and $\hat{z}$ directions, the maximum $n_{3}$ for the $P$-region is set equal to $M_{x}$ since the dimensions and materials match. For the remaining modes, a cut-off frequency $f_{c}$ is found from the highest order mode in the aperture, which sets the remaining $n$-indices $n_{1}$ and $n_{2}$ in the $P$-region as $N_{i}=2 f_{c} z_{0} \sqrt{\mu \varepsilon_{i}}\left[36\right.$. Finally, $V$ - and $C$-modes are chosen by including all $k_{z_{c, v}}$ that are equal to or less than the the maximum $k_{z_{a}}$, or the highest order mode [70].

With multiple simulations the convergence of the absolute values was determined for the resonances used for liquid measurements. The results can be seen in Figures $4.17 \mathrm{a}$ and $4.17 \mathrm{~b}$ for the $\mathrm{TE}_{221}$ mode, and in Figures $4.18 \mathrm{a}$ and $4.18 \mathrm{~b}$ for the $\mathrm{TE}_{521}$ mode. Only odd $M_{x}$ are necessary because of concentricity of the waveguide and aperture, and mid-height sidewall location of aperture. As can be seen, $f_{0}$ and $Q$ are converging with more aperture modes. For $N_{y} \geq 3$ there is not much variation in the results, so an extrapolation, in red, is made off of $N_{y}=2$. This is assuming that 
the offset between the $N_{y}=2$ and $N_{y}=5$ curves are the same for $M_{x} \geq 7$, which seems reasonable given the trends of the other curves. With this extrapolation, a final converged value is estimated, designated by the dashed blue line. To save computation time and memory, instead of using modes up to $M_{x}=13$ and $N_{y}=5$, the lower order-combination of $M_{x}=7$ and $N_{y}=2$ was chosen for the proximity of its results to the converged value.

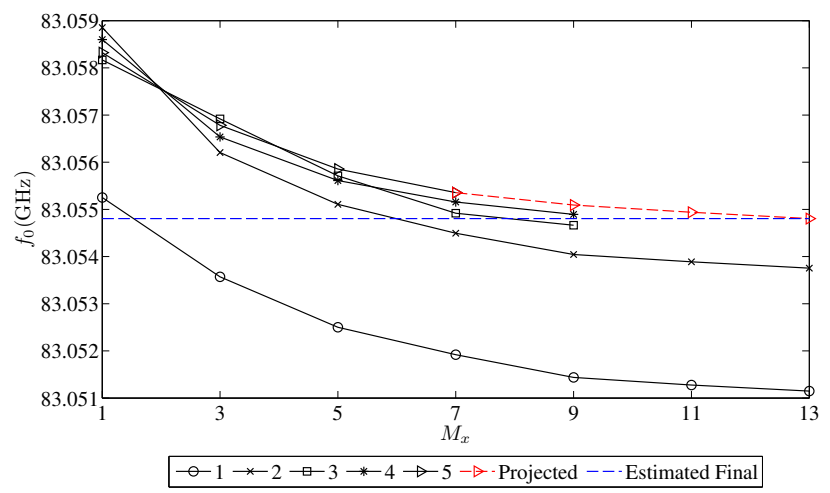

(a) Resonant frequency

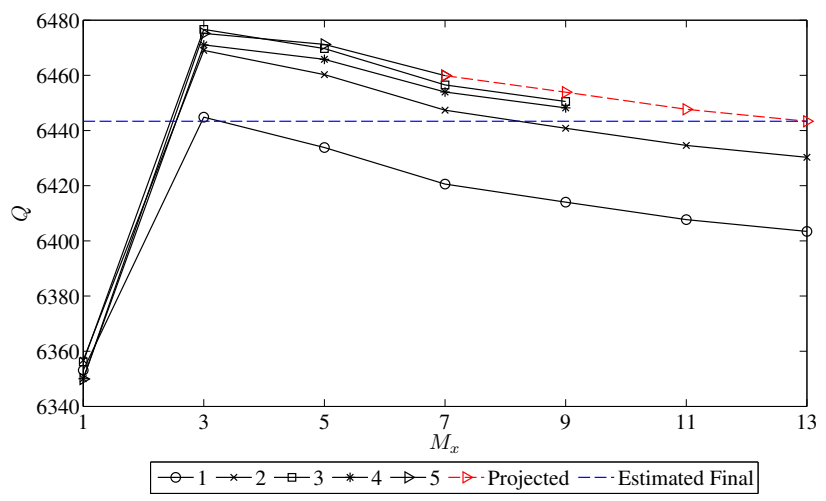

(b) Quality factor

Figure 4.17: Convergence of $\mathrm{TE}_{221}$ resonant frequency and Q-factor, over increasing aperture index $M_{x}$. Each curve is a unique value of $N_{y}$. The combination of $M_{x}=7$ and $N_{y}=2$ is within $1 \mathrm{MHz}$ of the estimated converged $f_{0}$ and within 10 of the estimated converged $Q$.

\subsubsection{Aperture approximation}

As discussed in $\$ 2.3 .4$, the inner product integrals at the aperture-cavity boundary must be numerically integrated. But since the aperture width is much smaller than the curvature of the cavity, a small-angle approximation can be used, which is explicitly shown in A.6.2. A comparison of the 


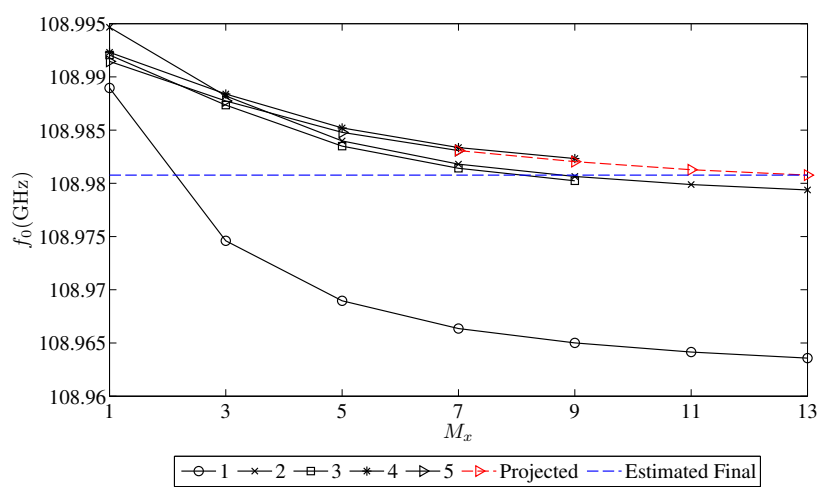

(a) Resonant frequency

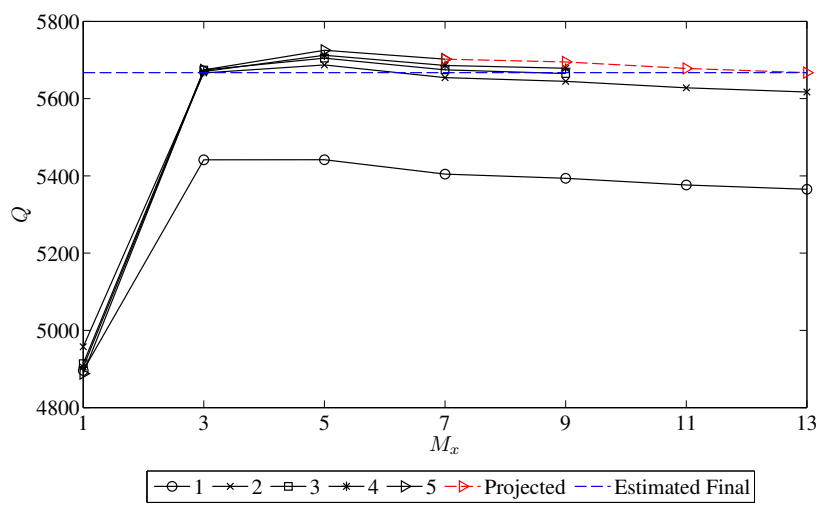

(b) Quality factor

Figure 4.18: Convergence of $\mathrm{TE}_{521}$ resonant frequency and Q-factor, over increasing aperture index $M_{x}$. Each curve is a unique value of $N_{y}$. The combination of $M_{x}=7$ and $N_{y}=2$ is about $1 \mathrm{MHz}$ off of the estimated converged $f_{0}$ and about 10 off of the estimated converged $Q$.

resonant frequencies and quality factors between the small-angle approximate $\phi$-integral and the numerical integral is shown in Figure 4.19. With the approximation, there is an error of about $400 \mathrm{kHz}$ in $f_{0}$ and 20 in $Q$ for the $\mathrm{TE}_{221}$ mode, and $2.5 \mathrm{MHz}$ and 55 for the $\mathrm{TE}_{521}$ mode.

\subsection{Dimensional measurements}

Dimension tolerances need to be set for the fabrication of the cavity block and the tube; however, these can produce a significant variation in the results, particularly in the resonant frequency with regards to the cavity radius. Dimensions were measured at a temperature different from data collection. So when running the simulations, the dimensions of the block are adjusted in accordance 


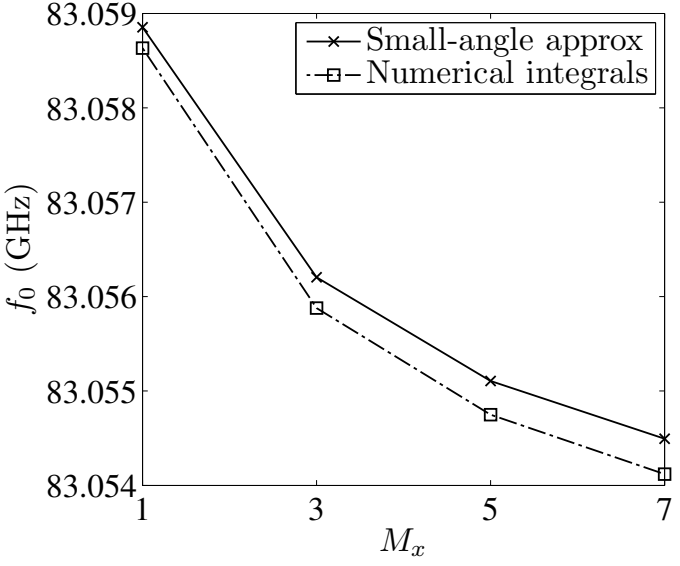

(a) Resonant frequency

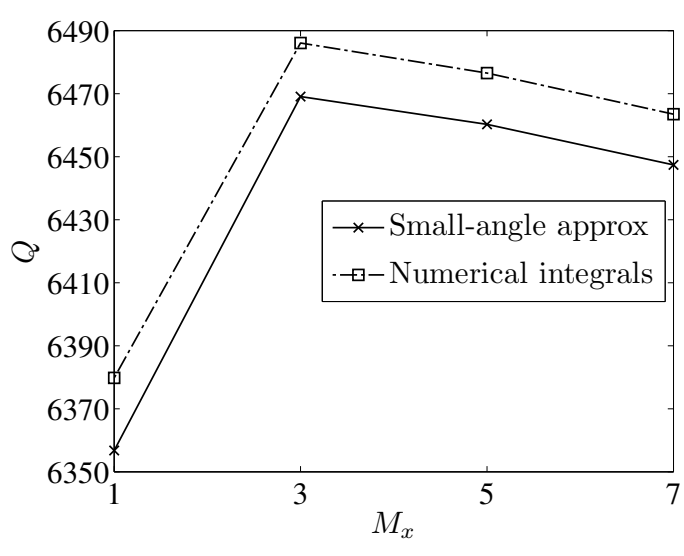

(b) Quality factor

Figure 4.19: Comparison of small-angle approximation vs. numerical integrals for the $\mathrm{TE}_{221}$ mode, over increasing $M_{x}$, for $N_{y}=2$. There is an error of about $400 \mathrm{kHz}$ in $f_{0}$ and 20 in $Q$.

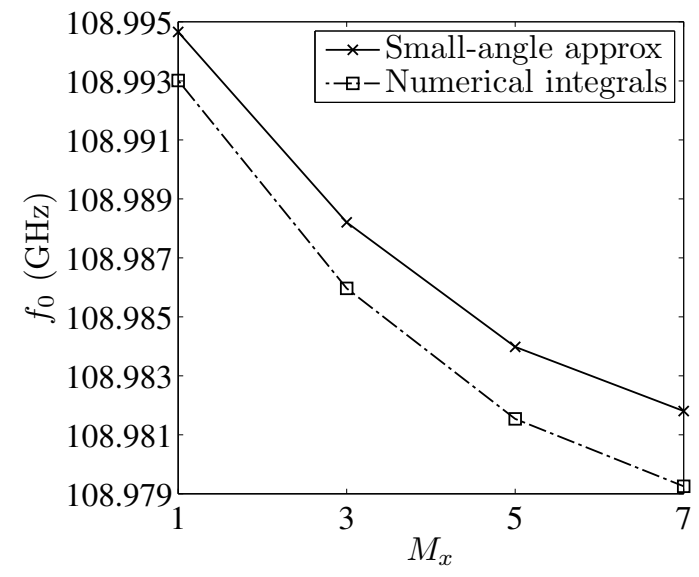

(a) Resonant frequency

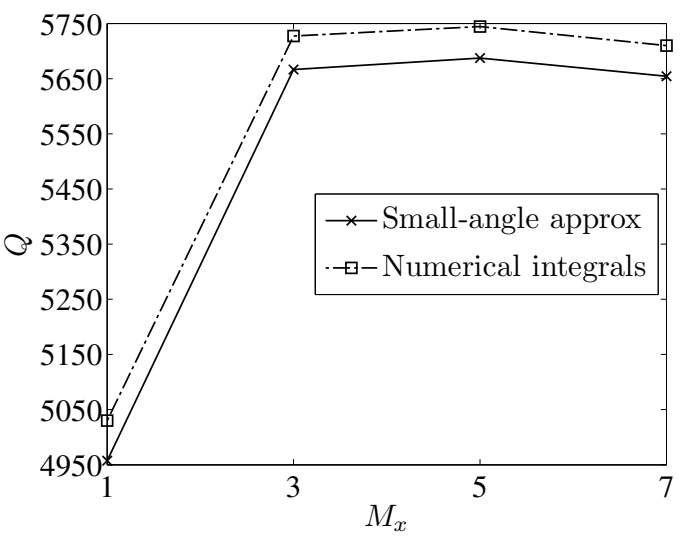

(b) Quality factor

Figure 4.20: Comparison of small-angle approximation vs. numerical integrals for the $\mathrm{TE}_{521}$ mode, over increasing $M_{x}$, for $N_{y}=2$. There is an error of about $2.2 \mathrm{MHz}$ in $f_{0}$ and 55 in $Q$.

to the temperature difference.

Most dimensions are measured by independent means, while the rest that cannot be easily measured are estimated from resonant frequency data. For these, a phase fit is done for each sweep, which is an average of 8 or 16 sweeps, so that the resonant frequency and quality factor are extracted. Then, a two-dimensional fit is performed with the modeling, using the LevenbergMarquardt method 76]. To compute the Jacobian, forward differences are used. 


\subsubsection{Cavity block}

Block dimensions that were measured are shown in Figure 4.21. The lateral dimensions $l$ and $y$ of the aperture were measured with a microscope that has a calibrated camera, while the depth $x$ of the apertures were measured with a white light interferometer. However, it was difficult to use the interferometer to image both the top and the bottom cavity faces in order to determine the depth $h$, which is nominally $1.27 \mathrm{~mm}$. Instead, a gauge normally used to measure the thickness of wafers for lapping was used. Because symmetry was assumed in the modeling, the dimensions for both apertures and waveguide were averaged into a single value. Measured and averaged dimensions are shown in Table 4.5. The uncertainty of $u_{2 h}$ is for both block halves and is found as

$$
u_{2 h}=\sqrt{u_{h}^{2}+u_{h}^{2}}
$$

where

$$
u_{h}=\sqrt{0.00011^{2}+0.00014^{2}}
$$

in which 0.00011 is the standard uncertainty of repeated gauge measurements and 0.00014 is a Type B error that reflects the $0.0005 \mathrm{~mm}$ precision of the gauge stand, which was calculated as 77

$$
\frac{0.00025}{\sqrt{3}}=0.00014
$$

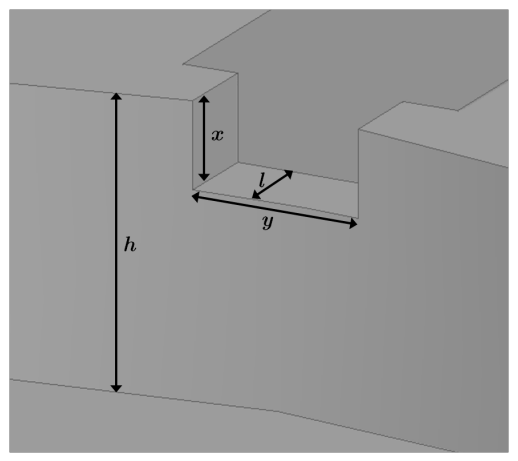

Figure 4.21: Key of block dimensions that were independently measured. 
Table 4.5: Measured cavity dimensions

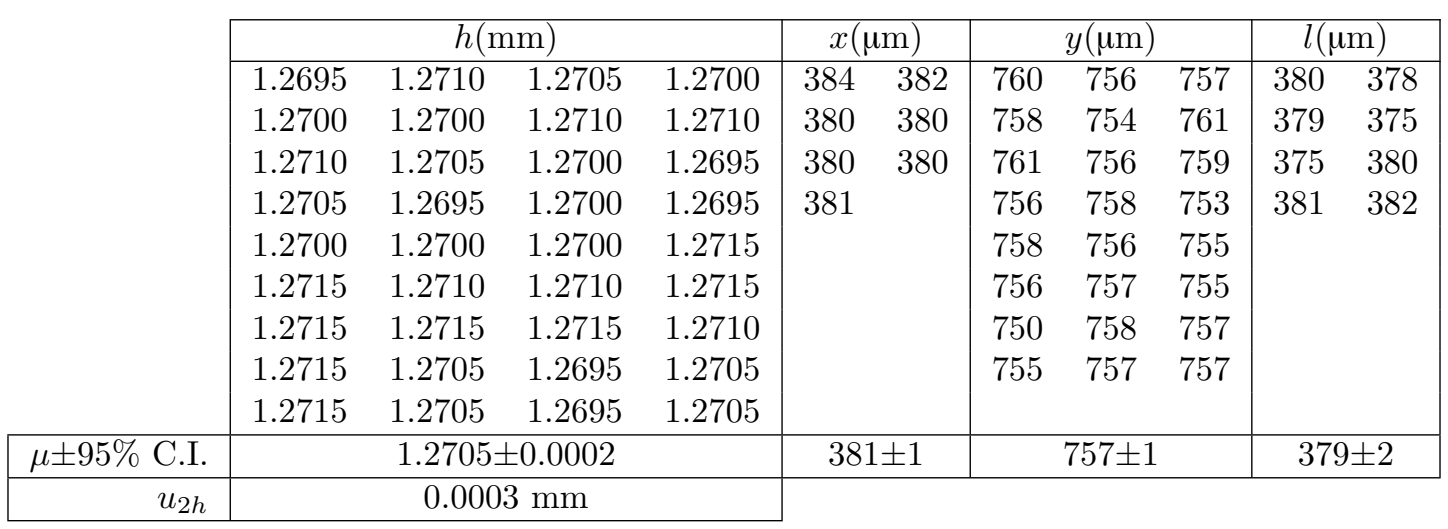

When measuring the dimensions, it was not realistically possible to ensure that the block was at the same temperature as when measured on the network analyzer. Additionally, multi-temperature measurements of permittivity, which are desirable for liquids, would be cumbersome. Instead, the temperature at which the dimensions were measured was recorded. Then in the modeling, when a comparison is made to measured $S_{21}$ data, the block dimensions were scaled by the linear coefficient of thermal expansion for aluminum, which constitutes close to all of the block.

Validation of this is shown in Table 4.6, where the cavity radius and conductivity was first tuned to match the $26^{\circ} \mathrm{C}$ measurement. Then this radius and the measured dimensions were increased to correspond with the expansion of $2^{\circ} \mathrm{C}$; the subsequent simulation matches very closely with the measurement of the cavity at $28^{\circ} \mathrm{C}$. The resonant frequencies match to a precision of $100 \mathrm{kHz}$, and the quality factors match within the error.

Table 4.6: Measured vs. simulated of temperature change ${ }^{1}$.

\begin{tabular}{|l|l|l|l|}
\hline \multicolumn{2}{|c|}{} & $f_{0}(\mathrm{GHz})$ & $Q$ \\
\hline \multirow{2}{*}{$29.0^{\circ} \mathrm{C}$} & Measured & $102.47167 \pm 0.00002$ & $2762 \pm 2$ \\
& Simulated & 102.47164 & 2759 \\
\hline \multirow{2}{*}{$29.1^{\circ} \mathrm{C}$} & Measured & $102.47147 \pm 0.00005$ & $2761 \pm 9$ \\
& Simulated & 102.47140 & 2759 \\
\hline \multirow{2}{*}{$30.0^{\circ} \mathrm{C}$} & Measured & $102.46927 \pm 0.00008$ & $2764 \pm 4$ \\
& Simulated & 102.46928 & 2759 \\
\hline \multicolumn{1}{|l}{} \\
\multicolumn{1}{|l}{$95 \%$ confidence intervals }
\end{tabular}

As for the cavity radius, it is difficult to measure this precisely, so this value is determined from resonant frequency measurements of the empty cavity. Presumably, a radius found from a fit to 
one resonance should be valid for another. To test this, three adjacent resonances were measured simultaneously in a single day. As shown in Figure 4.22 the measured resonant frequencies (black dashed lines) were compared to various modeled cavity radii and permittivity values for air (symbols). It can be seen that there is no combination of values that match all three resonances, although the results for $\mathrm{TE}_{021}$ and $\mathrm{TE}_{221}$ track closely. A possible explanation for this is if the two apertures are not directly across from each other, but instead are separated by an angle that is not $180^{\circ}$. An alternate angular offset disturbs the excitation of the mode and thus alters its field pattern in the cavity. Since the $\mathrm{TE}_{511}$ mode has the most lobes in the azimuthal direction, it will be the most sensitive to a change in coupling angular offset among these three modes. This contrasts to the $\mathrm{TE}_{021}$ mode, whose field pattern in $\hat{\phi}$ is constant. Various offset angles were tested using a simpler model of a lossless cavity without an inlet hole, with a method by Melloni that allows for a variable offset angle between the apertures [60]. As the results show in Figure 4.23 , a change of a few degrees produces a $2 \mathrm{MHz}$ decrease in the $\mathrm{TE}_{511}$ resonant frequency, while the $\mathrm{TE}_{221}$ mode is only perturbed by a few hundred kilohertz and the $\mathrm{TE}_{021}$ is barely disturbed. Therefore, if an angular offset were used in the simulations of Figure 4.22 , the modeled $\mathrm{TE}_{511} f_{0}$ would decrease and more closely match the measured $f_{0}$, with cavity conditions that are more consistent with the other two modes. However, since the angular offset for the inhomogeneous four-port model is fixed, the cavity radius is estimated to the measurement of the empty cavity.

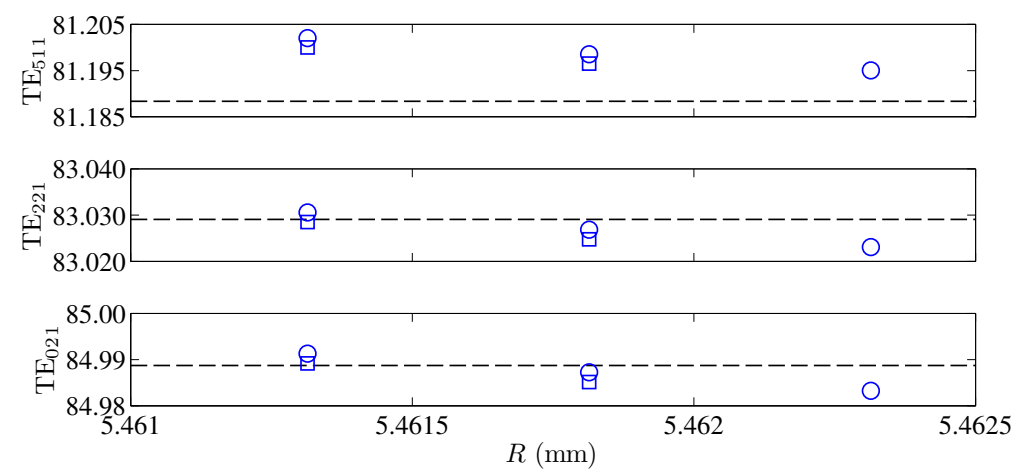

Figure 4.22: Simulated conditions of three resonances compared to measurements. The black dashed lines are the measured $f_{0}, R$ is the cavity radius, $\square: \varepsilon_{a i r}=1.0006$, o: $\varepsilon_{a i r}=1.00055$. Change in height shifts $f_{0}$ equally for all modes. 


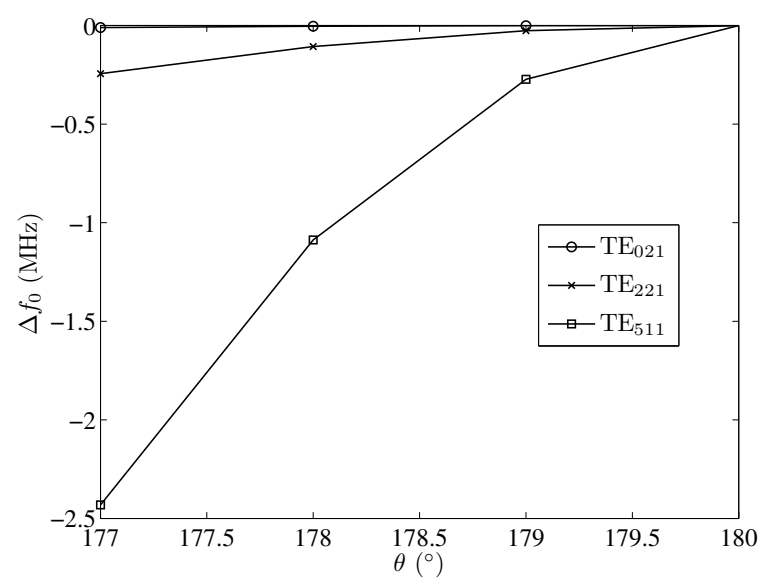

Figure 4.23: Sensitivity of $f_{0}$ to angle offset between apertures. Nominal dimensions. For the $\mathrm{TE}_{511}$ mode, which has the most azimuthal lobes, $f_{0}$ decreases by more than $2 \mathrm{MHz}$ for $3^{\circ}$ change in the angle.

\subsubsection{Tube}

The fused quartz tubes used for measurements have a manufacturer-specified $10 \%$ tolerance of the dimensions, which can produce a significant variation in the simulated resonant frequency for a mode with strong central electric fields. Thus, the inner and outer radii are measured for each tube.

The inner radius is found from weighing the tube filled with water. First, the scale was zeroed by weighing the empty tube. Then, the tube was partially filled with water, forming a column whose length $h$ is measured with a caliper. The final step was to measure the mass $m$ of the water-filled tube. Using the volume formula for a cylinder and the well-known density of water $\rho$, the inner radius $r$ can be found as

$$
r=\sqrt{\frac{m}{\rho \pi h}} .
$$

With repeated readings, the inner radius is determined by using a linear least-squares fit; for one of the sets of data being analyzed, this fit for the tube is shown in Figure 4.24 .

As for the outer radius, using a caliper was considered, but tightening of the caliper screw could compress the tube, and its resolution is only $20 \mu \mathrm{m}$. So, it was attempted to measure its outer diameter with the microscope. A photograph for same the tube of Figure 4.24 is shown in Figure 4.25 from which an average outer diameter value of $1009 \mu \mathrm{m}$ can be estimated. This outer diameter and the inner radius of Figure 4.24 were inputted into the modeling, along with the permittivity for fused 


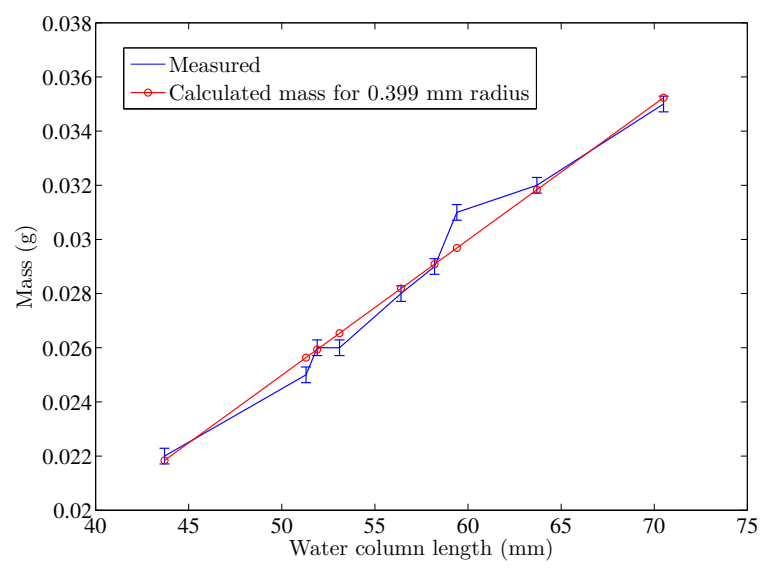

Figure 4.24: Least-squares fit of tube inner radius.

quartz, which was averaged from the higher frequency literature data in Figures 4.26 . The measured and modeled $f_{0}$ are shown in Table 4.7, and there is nearly a $7 \mathrm{MHz}$ difference, which is significant. If the permittivity is increased to $\varepsilon^{\prime}=4.1$, the simulated $f_{0}$ matches more closely, but compared to the literature data, this value is unreasonable. Instead, the outer diameter can be estimated by fitting the simulated resonance to the measured, in which case it is $1022 \mu \mathrm{m}$ and within the $10 \%$ tolerance. Also, the $1 \%$ difference of $12 \mu \mathrm{m}$ is more plausible, particularly if the widest part of the tube is not within focus.

Finally, the loss of the tube is estimated to the measured resonance, simultaneously with the outer diameter. From a survey of literature permittivity data for fused silica, which is a material very similar to fused quartz yet measured more extensively, the loss varied more widely and increased with greater frequency, even linearly in a single study [4]. Additionally, the loss will be sensitive to any impurities, such as water content 79,80$]$.

Table 4.7: Measured and Simulated Tube $f_{0}$

\begin{tabular}{|r|l|c|c|}
\hline & $f_{0}$ & $\varepsilon_{r}^{\prime}$ & O.D. $(\mu \mathrm{m})$ \\
\hline Measured (95\% C.I.) & $82.940439 \pm 0.000043$ & - & - \\
\hline \multirow{2}{*}{ Simulated } & 82.946763 & 3.79 & 1009 \\
& 82.941473 & 4.1 & 1009 \\
\hline Fitted & 82.940437 & 3.79 & 1022 \\
\hline
\end{tabular}




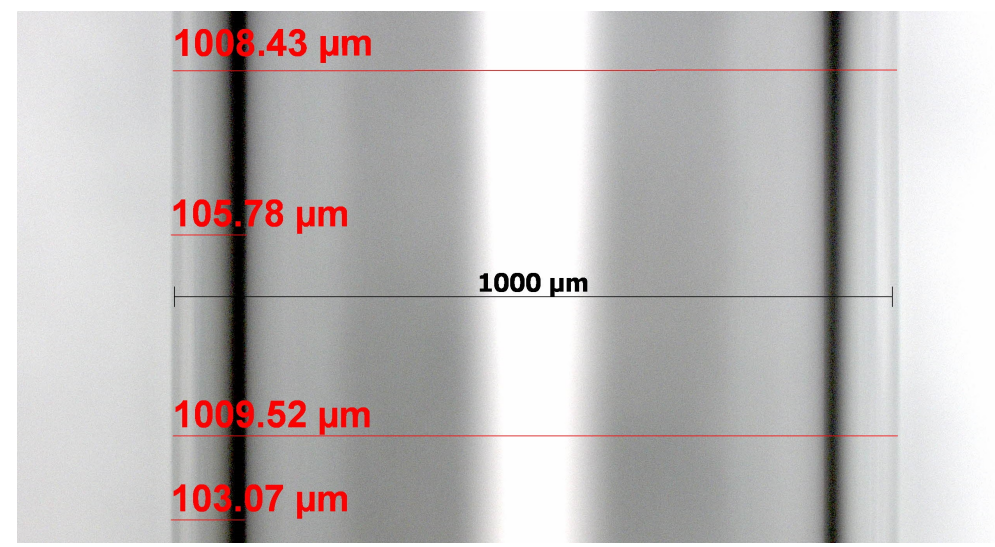

Figure 4.25: Microscope method for outer diameter measurement of tube.

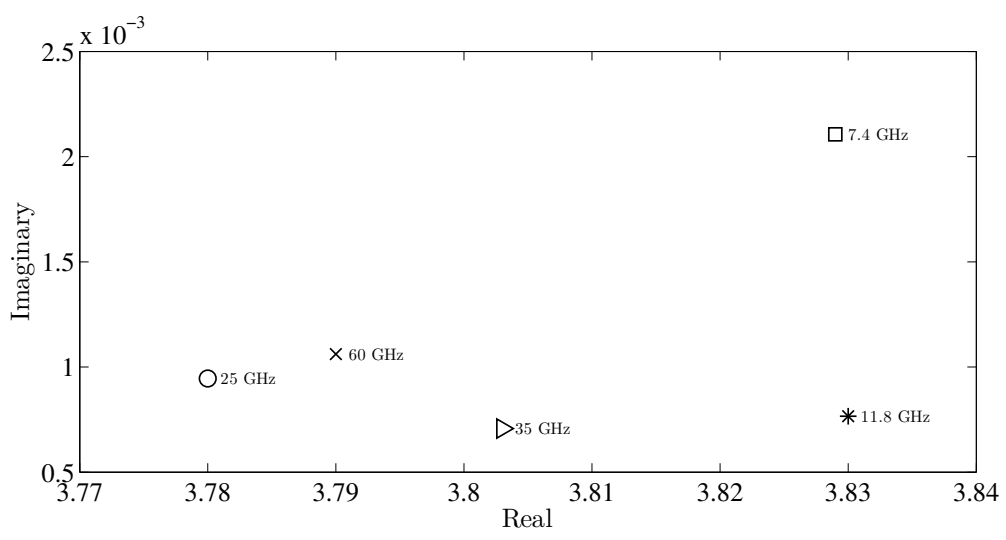

Figure 4.26: Real and imaginary permittivity values of fused quartz from literature 81 85. The value of 3.79 was selected for $\varepsilon^{\prime}$ as an average of the higher frequency data.

\subsection{Liquids}

Initially raw data of the cavity, tube, and methanol were taken with all of the resonances, in order to confirm the predicted sensitivities based on the modeling. A few modes of varying sensitivities were chosen to take calibrated measurements and test the method.

For each day of liquid measurements, the effective cavity radius and conductivity were found by fitting the modeling to the averaged resonant frequency and quality factor measurements of the empty cavity, using the Levenberg-Marquadt method. The same procedure was used with the tube data to determine its outer diameter and loss. Fitting values were considered converged if they were within the uncertainty of the data. Simulated $S$-parameters of the liquids were found either from 
fits or values used from the literature.

\subsubsection{Medium sensitivity}

The first mode measured was the $\mathrm{TE}_{221}$ mode, whose field plot is shown in Figure 4.27 . All of the field plots were generated in MATLAB using the modeling. The strongest lobes of the field are located outside of the nominal $0.5 \mathrm{~mm}$ radius tube, but there is still some interaction between the tube and lower strength fields. A comparison of the measured and simulated $S_{21}$ is shown

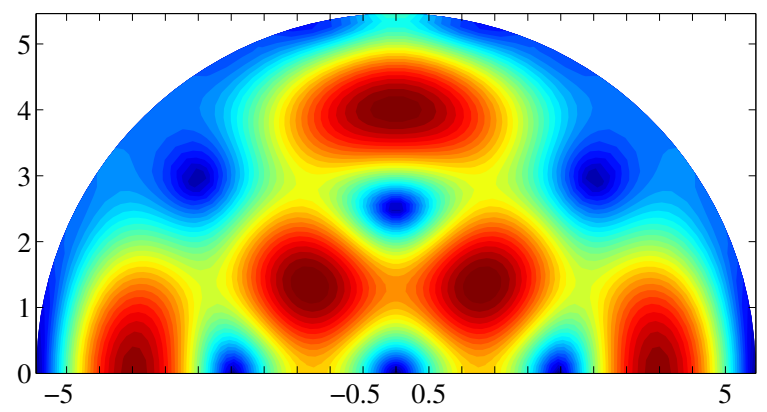

Figure 4.27: Electric field plot of $\mathrm{TE}_{221}$ mode. Axes in mm. Weaker fields interact with the $0.5 \mathrm{~mm}$ tube. Aperture coupling can be seen at $\phi=90^{\circ}$.

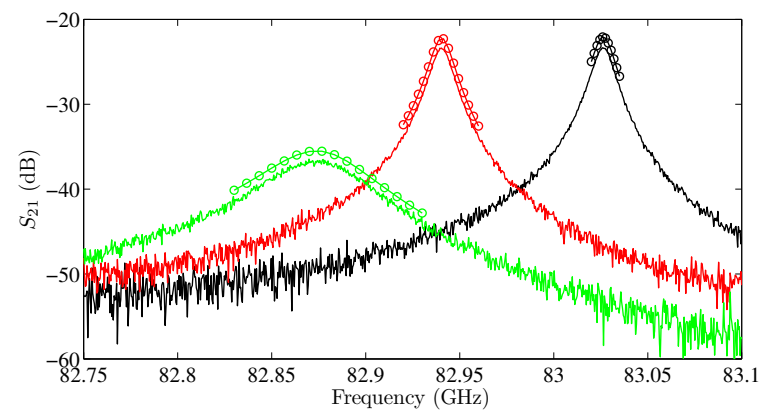

Figure 4.28: Comparison of measured and simulated Methanol I results. Black: empty cavity, $\mathrm{R}_{c}=5.4614 \mathrm{~mm}, \sigma=3.79 \times 10^{7} \mathrm{Sm}^{-1}$. Red: tube, $\mathrm{R}_{b}=511 \mu \mathrm{m}, \varepsilon_{2_{r}}^{\prime \prime}=0.003$. Green: methanol, $\varepsilon_{r}=5.0-j 2.7$.

in Figure 4.28. The electric conductivity is close to that of bulk gold $\left(\sigma=4.1 \times 10^{7} \mathrm{Sm}^{-1}\right)$, but is expected to be less since the block is merely plated with gold. It can be seen that the modeling captures the decreasing $S_{21}$ magnitude due to the dielectric losses, but there is an approximately $1 \mathrm{~dB}$ difference in magnitude. The coupling levels were consistent across multiple days, to within 
$0.5 \mathrm{~dB}$, even when the resonant frequencies shifted. Thus, this magnitude difference is systematic and lies with the modeling. It was found that with more modes $\left|S_{21}\right|$ decreases, so higher convergence would align the magnitudes more closely. Another cause could be asymmetric coupling, namely one aperture being smaller or longer than the averaged value.

From running simulations of multiple values of permittivity, it was deemed that this mode was sensitive enough so that the permittivity of methanol could be determined at a precision comparable to literature values; a more in-depth analysis is shown in $\$ 4.6$.

\section{Water}

In addition to methanol, water was measured with the $\mathrm{TE}_{221}$ mode; the data and fitted simulations for the empty cavity and tube can be seen in Figure 4.29 This set of data was taken over a month after the previous methanol data; the cavity radii are only $0.2 \mu \mathrm{m}$ different, and the conductivities are close to only $1 \%$ off. Different tubes were used, so the results are not comparable.

Water is considerably lossier than methanol, so the magnitude of $S_{21}$ has dropped by over $3 \mathrm{~dB}$. For reference, the permittivity data for water from previous studies is shown in Figure 4.30 7, 14. 16, 17, 25, 29, 86. The simulated $f_{0}$ for the nominal permittivity of water $\left(\varepsilon_{r}=10.2-j 16.6\right.$ 14 $)$ is close to that of the measurement, but $f_{0}$ and $Q$ differ by more than the measurement uncertainty. But, it is possible that on the wall of the tube a surface layer of water is being formed, and its properties differ from that of bulk water. This effect would be further magnified considering the fact that the surface layer would lie in the stronger part of the field, by examining the electric field plot in Figure 4.27

Attempts to use the fitting method to estimate a permittivity were unsuccessful. Manual simulations were done for various values, but $f_{0}$ and $Q$ could not be fitted simultaneously, i.e. $\chi^{2}>100$. Also, as demonstrated by the other permittivity simulations in Figure 4.29, the modeling for this mode is not as sensitive to changes in the permittivity for larger values. For a change of 2 in $\varepsilon_{r}^{\prime}$, only a $1 \mathrm{MHz}$ change is produced, whereas with the methanol fitting, a $3 \mathrm{MHz}$ change was observed for $\Delta \varepsilon_{r}^{\prime}=0.2$. Further work should be done to determine if this is a numerical problem with the modeling or if this decreased sensitivity with increasing permittivity would be seen experimentally. 


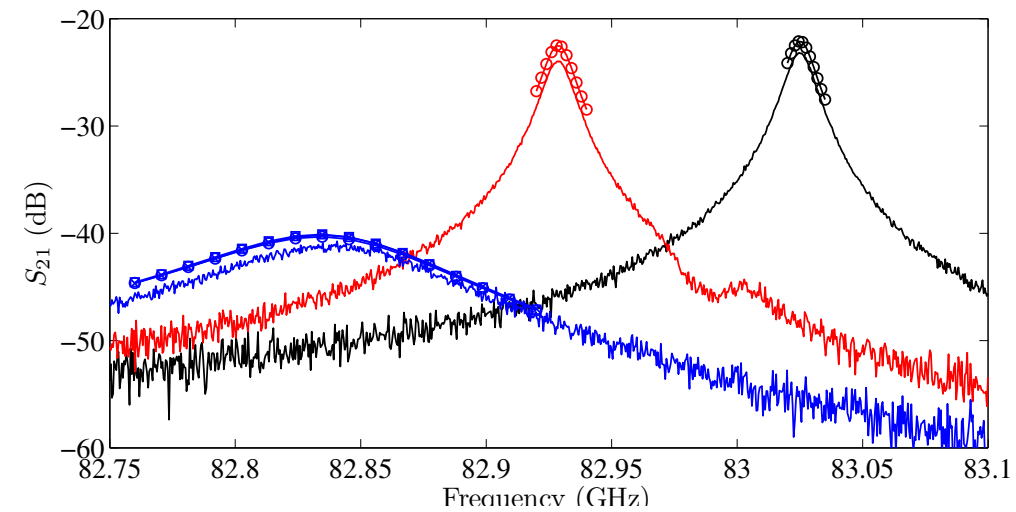

Figure 4.29: Measured and simulated water $26^{\circ} \mathrm{C} \mathrm{TE}_{221}$ results. Black: empty cavity, $\mathrm{R}_{c}=5.4616 \mathrm{~mm}$, $\sigma=3.75 \times 10^{7} \mathrm{Sm}^{-1}$. Red: tube, $\mathrm{R}_{b}=516 \mu \mathrm{m}, \varepsilon_{2_{r}}^{\prime \prime}=0.011$. Blue: water. Measured $f_{0}=82.8417 \pm 0.0002 \mathrm{GHz}, Q=848 \pm 3$. ०: $\varepsilon_{r}=10.2-j 16.6, f_{0}=82.8376, Q=823 . \times: \varepsilon_{r}=8-j 14$, $f_{0}=82.8387, Q=837$. $\square: \varepsilon_{r}=10-j 14, f_{0}=82.8374, Q=844$.

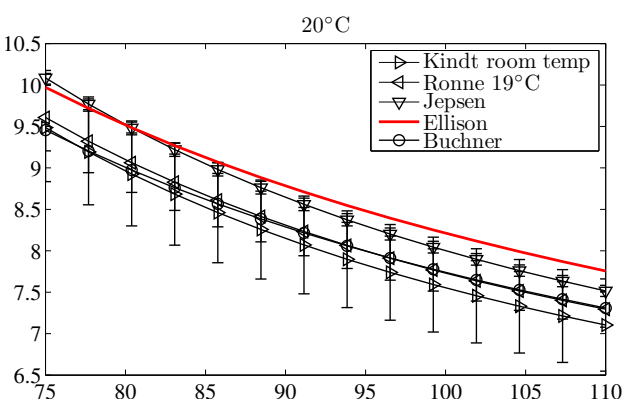

(a) $\varepsilon_{r}^{\prime}$

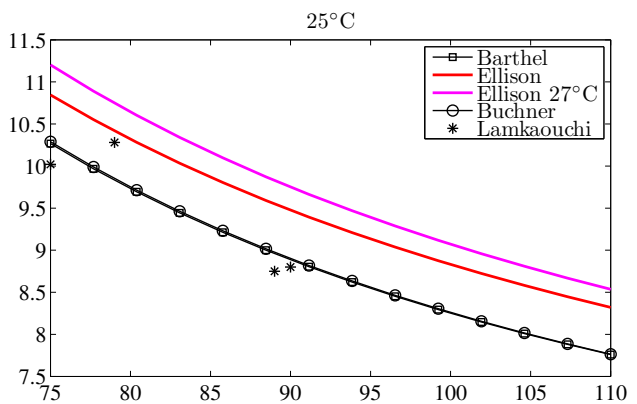

(c) $\varepsilon_{r}^{\prime}$

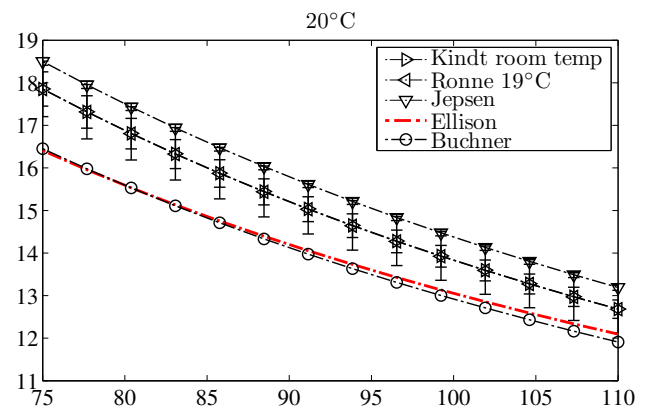

(b) $\varepsilon_{r}^{\prime \prime}$

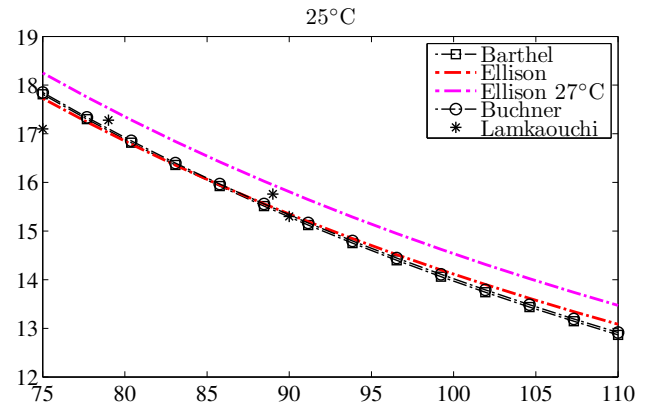

(d) $\varepsilon_{r}^{\prime \prime}$

Figure 4.30: Literature permittivity data of water.

\section{Smaller tube}

The next higher mode at $85 \mathrm{GHz}$ is the $\mathrm{TE}_{021}$ mode, which is more sensitive, as demonstrated by Figure 4.31. A methanol-filled $1 \mathrm{~mm}$ O.D. tube was so lossy that the resonance was rendered 
immeasurable, so a smaller $0.84 \mathrm{~mm}$ O.D. tube was tested. Data and simulation results are shown in Figure 4.32 While still close, the cavity radius and conductivity are a bit larger than with the $\mathrm{TE}_{221}$ mode. The liquid measurement was still considerably lossy, more than the water measurement.

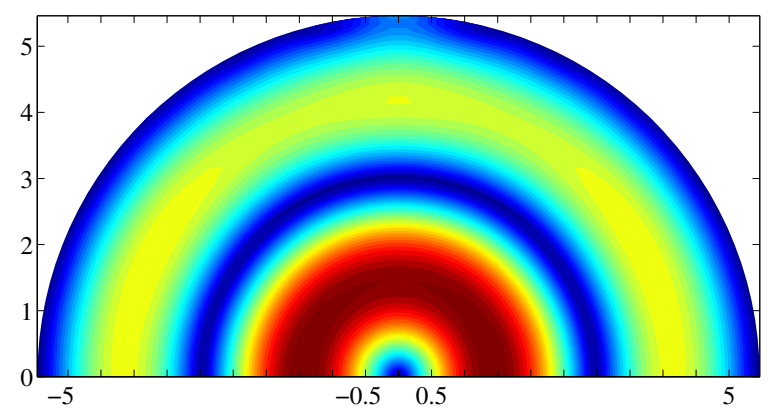

Figure 4.31: Electric field plot of $\mathrm{TE}_{021}$ mode. Axes in $\mathrm{mm}$. Stronger fields interact with the tube, versus the $\mathrm{TE}_{221}$ mode.

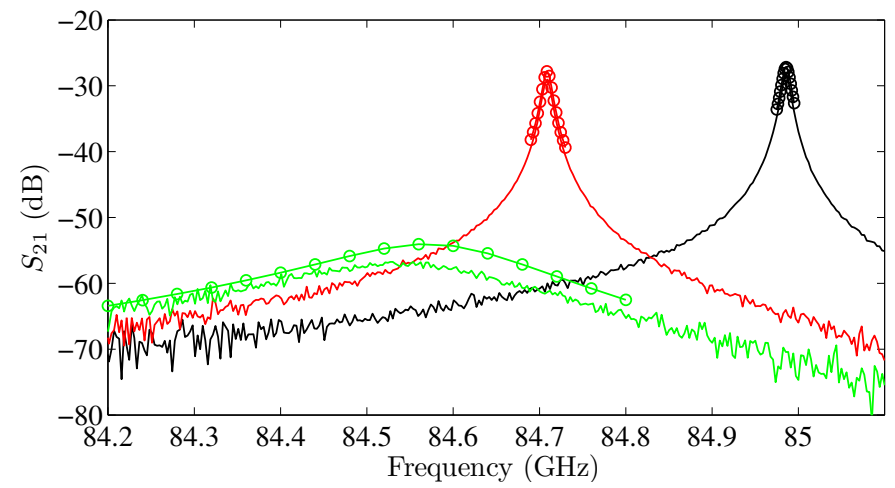

Figure 4.32: Measured and simulated water $\mathrm{TE}_{021}$ results. Black: empty cavity, $\mathrm{R}_{c}=5.4619 \mathrm{~mm}, \sigma=$ $3.80 \times 10^{7} \mathrm{Sm}^{-1}$. Red: tube, $\mathrm{R}_{b}=419 \mu \mathrm{m}, \varepsilon_{2_{r}}^{\prime \prime}=0.001$. Green: methanol. Measured $f_{0}=84.547 \mathrm{GHz}$, $Q=310$. ०: $\varepsilon_{r}=4.9-j 2.6, f_{0}=84.578, Q=371$.

\subsubsection{Low sensitivity}

Due to the high loss of water, more water measurements were done with a less sensitive mode, the $\mathrm{TE}_{521}$ mode seen in Figure 4.33 . Figure 4.34 shows data and the cavity fit, along with simulations for the tube and some water permittivity values. The fitted cavity radius is $5 \mu \mathrm{m}$ smaller than what was found with the lower order modes, and the conductivity is smaller as well. These results 


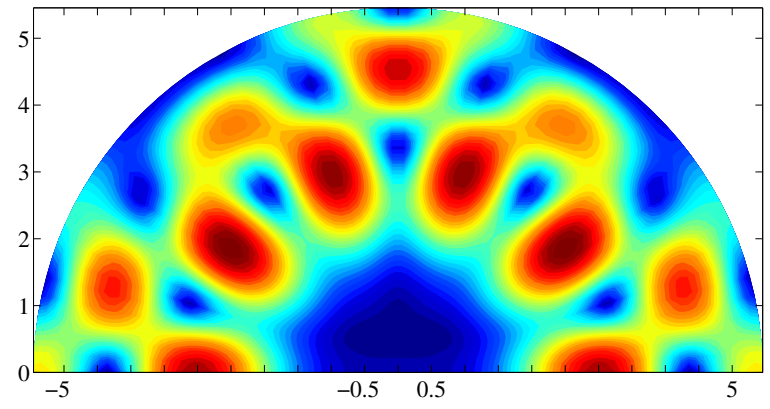

Figure 4.33: Electric field plot of $\mathrm{TE}_{521}$ mode. Axes in mm.

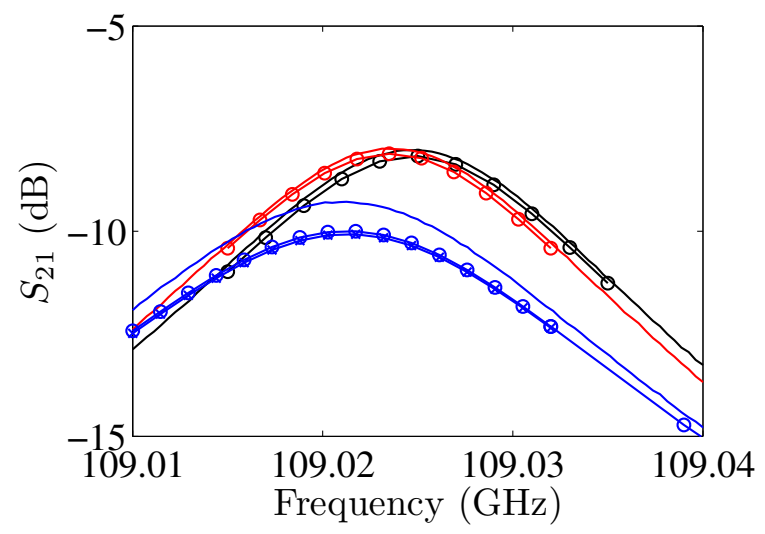

Figure 4.34: Measured and simulated water $27^{\circ} \mathrm{C} \mathrm{TE}_{521}$ results. Black: empty cavity, $\mathrm{R}_{c}=5.4562 \mathrm{~mm}$, $\sigma=3.2 \times 10^{7} \mathrm{Sm}^{-1}$. Red: tube, $\mathrm{R}_{b}=555 \mu \mathrm{m}, \varepsilon_{2_{r}}^{\prime \prime}=0.0009$. Blue: water. Measured $f_{0}=109.0213 \mathrm{GHz}, Q=4507 . \quad \circ: \varepsilon_{r}=8.6-j 13.6$ (nominal), $f_{0}=109.0215, Q=4239 . \quad \times: \varepsilon_{r}=$ $9.0-j 12.0, f_{0}=109.0215, Q=4204$.

were consistently seen across multiple days, so it is not likely that it is due to the data variation discussed in 4.2 .3 Likewise, use of the numerical integrals (Figure 4.20 that produce smaller $f_{0}$ would thus result in an even smaller estimated radius to compensate, since $f_{0}$ and the radius are inversely related. This is the same with the larger $Q$ values of the numerical integrals - an even lower conductivity would be estimated from the fit. Thus, it is believed that possible asymmetries or coupling angle offset might be the source of the error. It can also be observed that with a greater number of azimuthal lobes, the estimated radius and conductivity decrease.

The tube radius was set at the maximum value permitted by the tolerance; its $f_{0}$ is $70 \mathrm{kHz}$ larger than the measured, which is close but still greater than the uncertainty in the measured $f_{0}$. 
However, the resonant frequency for the nominal tube radius is also close, only $400 \mathrm{kHz}$ greater. This is indicative of the decreased sensitivity of this mode, as opposed to the MHz-scale changes seen in Table 4.7 for the $\mathrm{TE}_{221}$ mode.

A literature value for water of $\varepsilon_{r}=8.6-j 13.6$, based on Ellison's fit, was tested, along with another arbitrary value that is significantly different. Since the modeled results are very similar to each other, no permittivity estimation was done for this mode; in future, a larger tube is recommended. However, the simulation results are accurate, although an unexplained magnitude offset in $S_{21}$ appears, whose behavior is the opposite of what was seen with the lower order modes.

\subsubsection{Mode-mixing}

While the tuning of the cavity radius to obtain permittivity has thus far seemed to be a plausible method, the changing estimated cavity radii that appear with the different azimuthal variations is problematic if there are two closely-adjacent modes that mix together. This was seen for measurements with the mode at $95 \mathrm{GHz}$, which is predominately the $\mathrm{TE}_{711}$ mode but is affected by the lower order $\mathrm{TE}_{131}$ mode. After the cavity radius fit, the simulated $f_{0}$ for the nominal tube radius was $8 \mathrm{MHz}$ lower than what was measured, as seen in Figure 4.35. If the radius is decreased by $10 \%$ to $450 \mu \mathrm{m}$, then it is only $1 \mathrm{MHz}$ less, but based on a caliper measurement this value was not realistic. This problem was explored by looking at the broadband response and plotting the electric

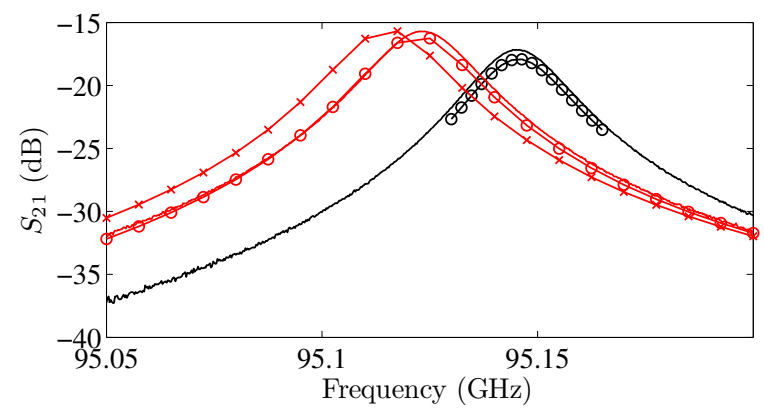

Figure 4.35: Measured and simulated $\mathrm{TE}_{711}$ results. Black: empty cavity, $\mathrm{R}_{c}=5.4576 \mathrm{~mm}, \sigma=$ $3.4 \times 10^{7} \mathrm{Sm}^{-1}$. Red: tube, o: $\mathrm{R}_{b}=450 \mu \mathrm{m}, \times: \mathrm{R}_{b}=500 \mu \mathrm{m}$.

fields. The resonances $\mathrm{A}$ and $\mathrm{C}$ of the simulation in Figure 4.36 are for the $\mathrm{TE}_{711}$ mode, while resonances $\mathrm{B}$ and $\mathrm{D}$ are for the $\mathrm{TE}_{131}$ mode. The electric field plots for these four resonances are 
shown in Figure 4.37. In the empty cavity plots, the $\mathrm{TE}_{711}$ mode has additional weak lobes towards the center that appear to come from the $\mathrm{TE}_{311}$ mode; likewise, the field for the latter mode has slight ripples in the outermost lobe, which seem to correspond to the lobes of the whispering gallery $\mathrm{TE}_{711}$ mode. When the tube is added, the resonances are further separated from one another, and the field plots match more closely to their closed-form responses, seen back in Figure 3.2 Based on the previous results, the estimated cavity radius for the $\mathrm{TE}_{711}-\mathrm{TE}_{131}$ mixture could be larger than it would have been for a more isolated $\mathrm{TE}_{711}$ mode. Thus, when the tube is added, a simulated $f_{0}$ for a feasible tube radius is for, in effect, a different mode. If a smaller cavity radius had been estimated for an isolated $\mathrm{TE}_{711}$ mode, then the simulated tube $f_{0}$ would also shift up in frequency.

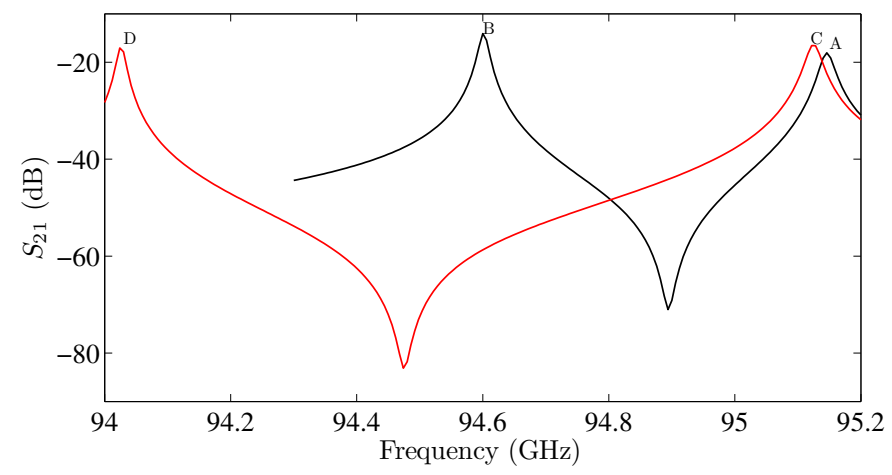

Figure 4.36: Simulated $\mathrm{TE}_{131}(\mathrm{~B}, \mathrm{D})$ and $\mathrm{TE}_{711}(\mathrm{~A}, \mathrm{C})$ results. Black: empty cavity, red: tube.

\subsection{Permittivity Data}

Permittivity data for methanol was obtained with the $\mathrm{TE}_{221}$ mode. The results for one day of methanol measurements can be seen in Table 4.8. This data was obtained at a lower convergence of $M_{x}=3$ and $N_{y}=1 ;$ at $M_{x}=7$ and $N_{y}=2$ the calculated permittivity is within $1 \%$, for retuned conductivity and radius.

The determined permittivity value is compared to literature values of various temperatures in Figure 4.38. Only studies that made measurements in the vicinity of $\mathrm{W}$-band were included. The $\mathrm{TE}_{221}$ value is close to the literature values, particularly the Saxton data if trend lines were extrapolated, but is more than $10 \%$ higher than the Barthel data, which is frequently cited and is for a temperature that is only $1^{\circ} \mathrm{C}$ different. Methanol was measured at $26^{\circ} \mathrm{C}$ because the temperature 


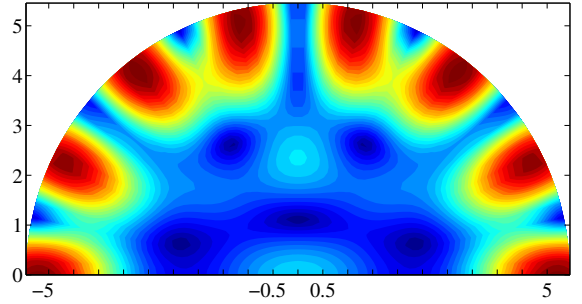

(a) $\mathrm{TE}_{711}$

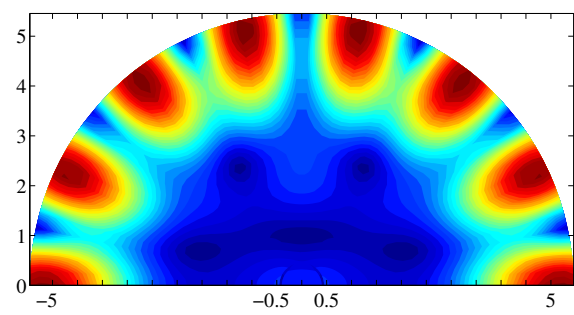

(c) $\mathrm{TE}_{711}$

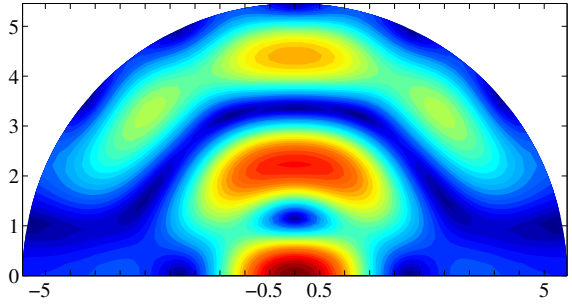

(b) $\mathrm{TE}_{131}$

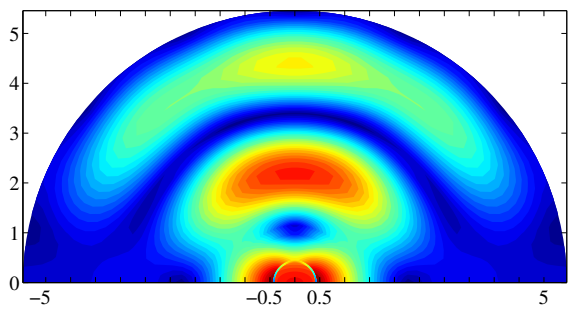

(d) $\mathrm{TE}_{131}$

Figure 4.37: Field plots of $\mathrm{TE}_{711}$ and $\mathrm{TE}_{131}$. Top row: cavity. Bottom row: tube. Mutual influence of $\mathrm{TE}_{131}$ and $\mathrm{TE}_{711}$ modes decreases with addition of tube.

Table 4.8: Methanol I Fitting Results, $83 \mathrm{GHz}$

\begin{tabular}{|l|c|c|c|c|}
\hline & Data & Parameter & Fitted & Nominal \\
\hline \multirow{2}{*}{ Empty } & $83.02636 \mathrm{GHz}$ & $R_{c}$ & $5.4614 \mathrm{~mm}$ & $5.4610 \mathrm{~mm}$ \\
& 6518 & $\sigma$ & $3.788 \times 10^{7} \mathrm{Sm}^{-1}$ & $4.1 \times 10^{7}(\mathrm{bulk} \mathrm{Au})$ \\
\hline \multirow{2}{*}{ Tube } & $82.94044 \mathrm{GHz}$ & $2 R_{b}$ & $1022 \mu \mathrm{m}$ & 1000 \\
& 6469 & $\varepsilon_{2_{r}}^{\prime \prime}$ & $3.4 \times 10^{-3}$ & $1 \times 10^{-3}$ \\
\hline Liquid & $82.87505 \mathrm{GHz}, 1410$ & $\varepsilon_{1_{r}}$ & $5.01-j 2.72\left(26^{\circ} \mathrm{C}\right)$ & $4.47-j 2.16\left(25^{\circ} \mathrm{C}\right)$ \\
\hline
\end{tabular}

of the block went above $25^{\circ} \mathrm{C}$ when it was connected to the frequency extension heads (which have amplifiers and mixers that require a power source, so heat is generated) and immersed in insulation. While the real parts of the $\mathrm{TE}_{221}$ and Kindt values are closer and within their uncertainties, the exact temperature was not specified. Although they compared their Debye parameters to those of Barthel, it is believed that their data was taken at a lower temperature, based on their water data of the same study (see Figure 4.30). The Jordan and Saxton data show how the permittivity of methanol changes with temperature in this frequency range, with the real and imaginary parts inversely and directly related to temperature, respectively. Thus, while the $\mathrm{TE}_{221} \varepsilon^{\prime \prime}$ value is feasible, it is possible that $\varepsilon^{\prime}$ is being overestimated. However, this is difficult to definitively say when the 
few measurements of methanol in this frequency range are contradictory, a point that is further exemplified by the spread of the data in Figure 4.30 . For instance, both parts of permittivity should be decreasing since this frequency range is between the second and third relaxations of methanol, according to the Debye fits of both Barthel and Kindt, which used far-infrared data and thus have estimations for the third relaxation. Given that fact, extrapolation of the Saxton and Jordan data to $83 \mathrm{GHz}$ would also be different from Barthel and Kindt.

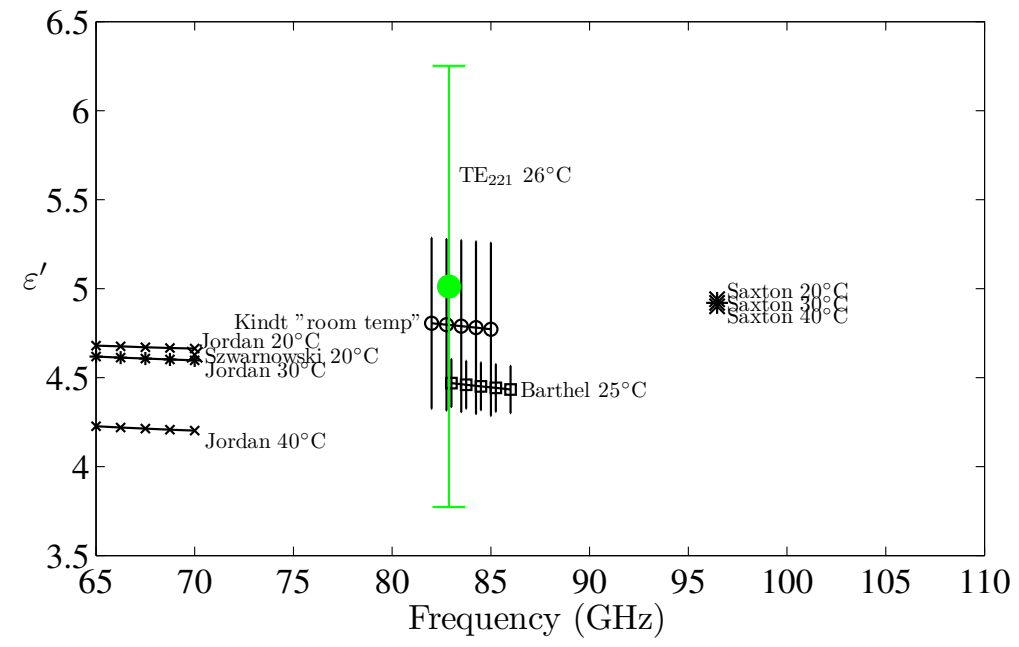

(a) Real part

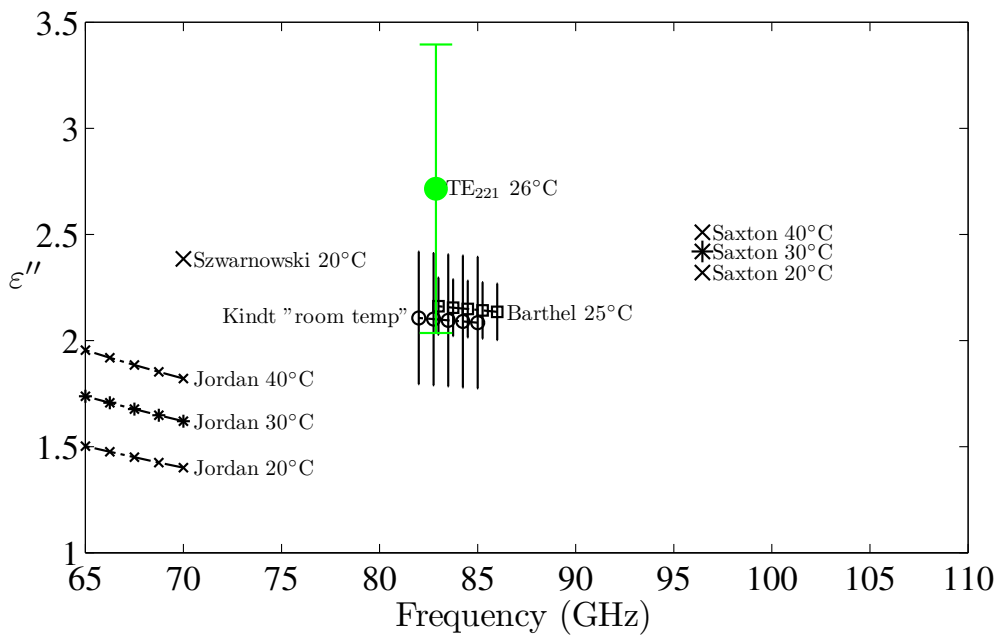

(b) Imaginary part

Figure 4.38: Comparison of methanol permittivity results to literature $7,29,87,89$. Multi-frequency curves are Debye functions. 


\subsubsection{Uncertainty}

The next thing to be addressed is the uncertainty, which was calculated using the propagation of uncertainty formula. All of the estimated parameters $y$ can be considered as a function $f$ of the measured dimensions or other known inputs $x_{i}$ as so

$$
y=f\left(x_{1}, x_{2}, \ldots\right)
$$

where $f$ is the mode-matching modeling of the resonator 70 . The propagation of uncertainty formula is

$$
u_{y}=\sqrt{\left(\frac{\partial f}{\partial x_{1}} u_{x_{1}}\right)^{2}+\left(\frac{\partial f}{\partial x_{2}} u_{x_{2}}\right)^{2}+\ldots}
$$

which is also the norm of the standard uncertainties, or the sensitivities multiplied by their uncertainties. These sensitivities are partial derivatives, which have to be found numerically with the forward difference formula

$$
\frac{\partial f}{\partial x} \approx \frac{f(x+\delta)-f(x)}{\delta}
$$

where $\delta$ is set at a small value. To compute these, each $x$ is altered by $\delta$ so that there is a significant change in $f_{0}$ and $Q$. Then, the fitting method finds $f(x+\delta)$. For the above methanol results, the calculated sensitivities and corresponding uncertainties are shown in Table 4.9. From simulations it was found that the resonances were not very sensitive to the aperture size nor to the inner radius of the tube, so these parameters were not included in the analysis. It can be seen that the liquid permittivity is the most sensitive to the tube outer diameter and the cavity radius and height. But, the former two dimensions are estimated and are also the most sensitive to the cavity height. In fact, if $u_{h}$ were lowered to $0.1 \mu \mathrm{m}$, then the uncertainties in $\varepsilon_{r}^{\prime}$ and $\varepsilon_{r}^{\prime \prime}$ are also reduced to a third of their former value, or about $8 \%$. This is lower than the $10 \%$ uncertainty in the Kindt data, which was determined by propagation of given uncertainties of the Debye parameters through the Debye formula. The accuracy of the Barthel data, on the other hand, was estimated as $3 \%$, but this value seemed to be based on deviations of the data from their Debye fit. Standard deviations for discrete permittivity data points were presented, but these were likely estimated from repeated measurements and did not examine dimensional uncertainties in their modeling 30]. 
Table 4.9: Uncertainty budget for $\mathrm{TE}_{221}$ methanol data

\begin{tabular}{|c|c|c|c|}
\hline $\mathbf{x}$ & $\mathbf{u}_{\mathbf{x}}$ & \multicolumn{2}{|c|}{ Standard Uncertainty } \\
\hline \multicolumn{4}{|c|}{ Empty } \\
\hline & & $R(\mu \mathrm{m})$ & $\sigma\left(10^{5} \mathrm{Sm}^{-1}\right)$ \\
\hline$h$ & $0.3 \mu \mathrm{m}$ & -0.65 & 0 \\
\hline$f_{0}$ & $0.6 \mathrm{MHz}$ & -0.08 & 0.06 \\
\hline$Q$ & 15 & 0.002 & 1.93 \\
\hline & & 0.66 & 1.93 \\
\hline \multicolumn{4}{|c|}{ Tube } \\
\hline & & $R_{b}(\mu \mathrm{m})$ & $\varepsilon_{2_{r}}^{\prime \prime} \times 10^{-3}$ \\
\hline$h$ & $0.3 \mu \mathrm{m}$ & -4.66 & 0.15 \\
\hline$R$ & $0.66 \mu \mathrm{m}$ & -4.79 & 0.15 \\
\hline$\sigma$ & $1.93 \times 10^{5} \mathrm{Sm}^{-1}$ & 0.02 & 0.77 \\
\hline$f_{0}$ & $0.6 \mathrm{MHz}$ & -0.57 & 0.014 \\
\hline$Q$ & 14 & 0.0009 & -0.72 \\
\hline & & 6.7 & 1.07 \\
\hline \multicolumn{4}{|c|}{ Liquid } \\
\hline & & $\varepsilon_{r}^{\prime}$ & $\varepsilon_{r}^{\prime \prime}$ \\
\hline$h$ & $0.3 \mu \mathrm{m}$ & -0.59 & -0.27 \\
\hline$R$ & $0.66 \mu \mathrm{m}$ & -0.53 & -0.25 \\
\hline$\sigma$ & $1.93 \times 10^{5} \mathrm{Sm}^{-1}$ & 0.001 & 0.003 \\
\hline$R_{b}$ & $6.7 \mu \mathrm{m}$ & -0.95 & -0.57 \\
\hline$\varepsilon_{2_{r}}^{\prime \prime}$ & $1.07 \times 10^{-3}$ & 0.001 & -0.002 \\
\hline$f_{0}$ & $0.6 \mathrm{MHz}$ & -0.07 & -0.03 \\
\hline$Q$ & 5 & 0.006 & -0.01 \\
\hline & & 1.24 & 0.68 \\
\hline
\end{tabular}

\subsubsection{Repeatability}

$\mathrm{TE}_{221}$ measurements of methanol at $26^{\circ} \mathrm{C}$ were repeated on a consecutive day, with a different and unused quartz tube. The results of set II can be seen in Table 4.10, with the set I results repeated for comparison. Because the tube outer diameter was considerably larger, which was also observed with the microscope and the caliper, the tube shifted the resonance further down in frequency, so the methanol measurements are $19 \mathrm{MHz}$ apart. Based on the Barthel Debye fit, the permittivity of methanol changes by less than $0.5 \%$ at these two frequencies. The I and II permittivity results are close, within $3 \%$, and are certainly within the uncertainty. The different losses could be sensitive to varying impurities in the methanol and its exposure to the ambient air of the lab, since it was being stored in a jar. In actuality, the set II data was measured the day before set I, so this could help explain the increased loss and decreased real part of the permittivity. 
Table 4.10: Methanol $\left(26^{\circ} \mathrm{C}\right), 83 \mathrm{GHz}$ : I vs. II Fitting Results

\begin{tabular}{|l||c|c||c|c|}
\hline Parameter & Data & I & II & Data \\
\hline$R_{c}$ & $83.02636 \mathrm{GHz}$ & $5.4614 \mathrm{~mm}$ & $5.4616 \mathrm{~mm}$ & $83.02528 \mathrm{GHz}$ \\
$\sigma$ & 6518 & $3.788 \times 10^{7} \mathrm{Sm}^{-1}$ & $3.789 \times 10^{7} \mathrm{Sm}^{-1}$ & 6520 \\
\hline $2 R_{b}$ & $82.94044 \mathrm{GHz}$ & $1022 \mu \mathrm{m}$ & $1050 \mu \mathrm{m}$ & $82.91887 \mathrm{GHz}$ \\
$\varepsilon_{2_{r}}^{\prime \prime}$ & 6469 & $3.4 \times 10^{-3}$ & $2.8 \times 10^{-3}$ & 6462 \\
\hline$\varepsilon_{1_{r}}$ & $82.87505 \mathrm{GHz}, 1410$ & $5.01-j 2.72$ & $5.07-j 2.63$ & $82.85638 \mathrm{GHz}, 1446$ \\
\hline
\end{tabular}




\section{Chapter 5}

\section{Conclusions and Future Work}

In this work, the use of a full-wave electromagnetic model of a cavity resonator to measure the permittivity of liquids was explored. For the first time, a four-port, inhomogeneous waveguide structure with built-in electric conductivity loss was analytically formulated and coded. This complex modeling allowed for the automatic inclusion of the resonator coupling effects, as well as flexibility with modeling the effect of the inlet hole for the liquid. A new waveguide resonator was designed, which included environmental controls for accurate and precise measurements. Over the course of an hour the data was shown to be very precise, as well as over consecutive days with equivalent mechanical connections. Methanol and water were measured at 83 and $109 \mathrm{GHz}$, and the modeling produced permittivity data for methanol that were moderately comparable to that of the literature. Uncertainties in the permittivity results were $25 \%$, which was attributable to uncertainty in the cavity height. The measurements for water were either too lossy or not sensitive enough to obtain meaningful data.

An uncertainty analysis was conducted for a day of methanol measurements and included the more influential sources of error. It was concluded that a majority of the uncertainty was due to the uncertainty in the cavity height. In light of this, the next resonator designed should instead have TM mode-excitation so that the $\mathrm{TM}_{n p 0}$ modes can be measured, since they are insensitive to the cavity height. The field plot of an example $\mathrm{TM}_{n p 0}$ mode is shown in Figure 5.1 in opposition to the $\mathrm{TE}_{221}$ mode that was measured. As with the TE-mode, moderate-strength fields are located within the inlet hole region. From repeated measurements, it was determined that the disassembly 


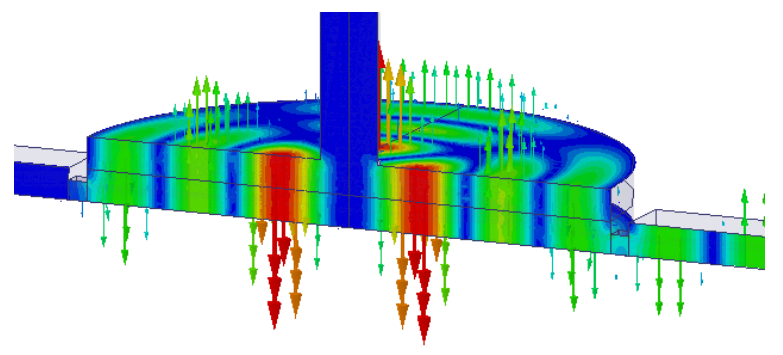

(a) $\mathrm{TM}_{230}$

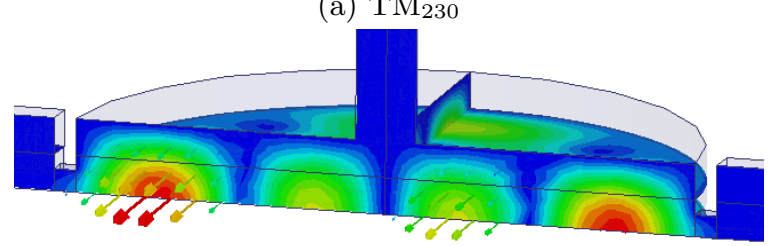

(b) $\mathrm{TE}_{221}$

Figure 5.1: HFSS field plots of TM and TE modes. The TM-fields are insensitive to the cavity height.

of the block was responsible for the largest variation in inter-day resonant frequencies, to which the cavity diameter is estimated. In future, it is recommended to not split the block directly through the waveguide but rather at either end of the cavity, as shown in Figure 5.2 While this should present no difficulty in creating the circular cylinder for the cavity region, the side-coupled aperture and waveguide will likely have larger corner radii and not be as rectangular. Since the fields for the input excitation, the $\mathrm{TE}_{10}$ mode, are mostly in the center, this should not have a huge effect, but as a worst case there are numerous papers on elliptical waveguide. For the split at the top of the block, the $E_{z}$ field must short at the cavity sidewall, so there must be good contact between the two halves.

Measurement of water was difficult with the available dynamic range of the network analyzer. This is less of a problem with newer VNAs and frequency extension head systems that provide more dynamic range, up to $100 \mathrm{~dB}$ in $\mathrm{W}$-band. To take the fullest advantage of this capability, a very low-loss material should be used to make the dielectric plug in the Nitrogen blocks, such as hydrocarbon foams.

Additionally for water, the properties of surface layers should be explored, and its effects should be taken into consideration in the new design, in particular the size of the tube and its relation to 


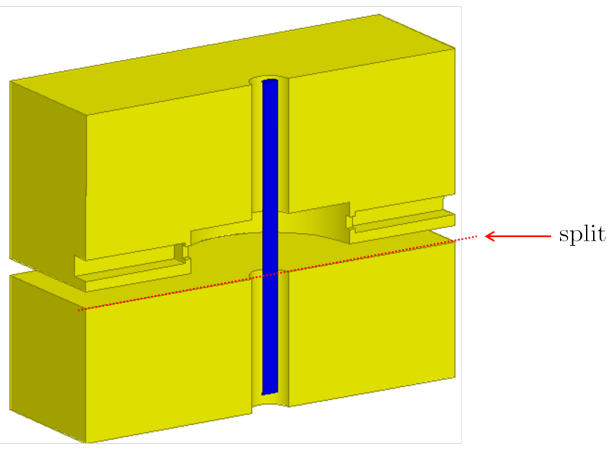

Figure 5.2: Cross-sectional view of recommended split for TM mode-excitation block.

the electric field.

The biggest challenge with the modeling was the computation of the inhomogeneous propagation constants. The root-search would sometimes converge to erroneous roots, so the method was made to ensure that all of the roots were found, and became computationally time-consuming. Instead, the very recent four-port ring network method is recommended, in which a four-port matrix is found for each concentric dielectric layer; the method also has the capability to include lossy metallic walls 90 . 


\section{Appendix A}

\section{Equations}

\section{A.1 Waveguide}

Fields solutions for waveguide start with the source-free, time-harmonic Helmholtz Equation, derivable from Maxwell's Equations [37]:

$$
\nabla^{2} \psi+k^{2} \psi=0
$$

where $\nabla^{2}$ is the Laplacian, $\psi$ is a vector potential, and $k$ is the wavenumber. For mode-matching, the functional form of the solutions to the Helmholtz equation are found by separating the problem into regions that can be solved more easily, i.e. regions of uniform waveguide with geometries that are definable by coordinate surfaces of a curvilinear coordinate system in which the Helmholtz equation is separable [54]. The measurement cavity is separable into rectangular and cylindrical regions, and their respective building blocks are rectangular and circular/parallel-plate waveguide, which are diagrammed in Figures A.1 and A.2. Fields in both types of waveguide are designated as either TE $(h)$, transverse electric, or TM $(e)$, transverse magnetic. The following relations, which are for lossless and homogeneous waveguide, are standard in advanced electromagnetic texts, but the notation used is mostly from Harrington and Balanis 36,37. In rectangular waveguide, the TE- 
and TM-wave potentials are

$$
\begin{aligned}
\psi_{m n}^{h} & =\cos \frac{m \pi x}{a} \cos \frac{n \pi y}{b} e^{-k_{z} z} \\
\psi_{m n}^{e} & =\sin \frac{m \pi x}{a} \sin \frac{n \pi y}{b} e^{-k_{z} z} \\
k_{z} & =\sqrt{\left(\frac{m \pi}{a}\right)^{2}+\left(\frac{n \pi}{b}\right)^{2}-k^{2}}
\end{aligned}
$$

where $m$ and $n$ are integers. From these wave potentials, the field components in each direction can be found with the following

$$
\begin{aligned}
E_{x}^{h} & =-\frac{\partial \psi^{h}}{\partial y} & H_{x}^{h} & =\frac{1}{j \omega \mu} \frac{\partial^{2} \psi^{h}}{\partial x \partial z} \\
E_{y}^{h} & =\frac{\partial \psi^{h}}{\partial x} & H_{y}^{h} & =\frac{1}{j \omega \mu} \frac{\partial^{2} \psi^{h}}{\partial y \partial z} \\
E_{z}^{h} & =0 & H_{z}^{h} & =\frac{1}{j \omega \mu}\left(\frac{\partial^{2}}{\partial z^{2}}+k^{2}\right) \psi^{h} \\
E_{x}^{e} & =\frac{1}{j \omega \varepsilon} \frac{\partial^{2} \psi^{e}}{\partial x \partial z} & H_{x}^{e} & =\frac{\partial \psi^{e}}{\partial y} \\
E_{y}^{e} & =\frac{1}{j \omega \varepsilon} \frac{\partial^{2} \psi^{e}}{\partial y \partial z} & H_{y}^{e} & =-\frac{\partial \psi^{e}}{\partial x} \\
E_{z}^{e} & =\frac{1}{j \omega \varepsilon}\left(\frac{\partial^{2}}{\partial z^{2}}+k^{2}\right) \psi^{e} & H_{z}^{e} & =0
\end{aligned}
$$

Similarly for circular and parallel-plate waveguide the potentials are

$$
\begin{aligned}
& \psi_{m n}^{h}=J_{m}\left(k_{\rho}^{h} \rho\right)\left\{\begin{array}{c}
\cos m \phi \\
\sin m \phi
\end{array}\right\} Z^{h}(z) \\
& \psi_{m n}^{e}=J_{m}\left(k_{\rho}^{e} \rho\right)\left\{\begin{array}{c}
\cos m \phi \\
\sin m \phi
\end{array}\right\} Z^{e}(z)
\end{aligned}
$$


where $m$ is an integer. The $z$-wavefunctions and wavenumbers for circular waveguide, which has a perfect electric conductor at $\rho=R$, are

$$
\begin{aligned}
k_{\rho}^{h} & =\frac{\chi_{m n}^{\prime}}{R} \\
k_{\rho}^{e} & =\frac{\chi_{m n}}{R} \\
k_{z}^{h, e} & =\sqrt{k^{2}-k_{\rho}^{h, e^{2}}} \\
Z^{h, e}(z) & =e^{-j k_{z}^{h, e} z}
\end{aligned}
$$

where $\chi_{m n}$ and $\chi_{m n}^{\prime}$ are the $n$th zeros of $J_{m}$ and $J_{m}^{\prime}$, respectively. For parallel-plate waveguide, which has perfect electric conductors at $z=0$ and $h$, the $z$-wavefunctions and wavenumber are

$$
\begin{aligned}
Z^{h}(z) & =\sin \frac{n \pi}{h} z \\
Z^{e}(z) & =\cos \frac{n \pi}{h} z \\
k_{\rho}^{h, e} & =\sqrt{k^{2}-\left(\frac{n \pi}{h}\right)^{2}}
\end{aligned}
$$

where $n$ is an integer. The fields can be found in a fashion similar to rectangular waveguide

$$
\begin{aligned}
E_{\rho}^{h} & =-\frac{1}{\rho} \frac{\partial \psi^{h}}{\partial \phi} & H_{\rho}^{h} & =\frac{1}{j \omega \mu} \frac{\partial^{2} \psi^{h}}{\partial \rho \partial z} \\
E_{\phi}^{h} & =\frac{\partial \psi^{h}}{\partial \rho} & H_{\phi}^{h} & =\frac{1}{j \omega \mu \rho} \frac{\partial^{2} \psi^{h}}{\partial \phi \partial z} \\
E_{z}^{h} & =0 & H_{z}^{h} & =\frac{1}{j \omega \mu}\left(\frac{\partial^{2}}{\partial z^{2}}+k^{2}\right) \psi^{h} \\
E_{\rho}^{e} & =\frac{1}{j \omega \varepsilon} \frac{\partial^{2} \psi^{e}}{\partial \rho \partial z} & H_{\rho}^{e} & =\frac{1}{\rho} \frac{\partial \psi^{e}}{\partial \phi} \\
E_{\phi}^{e} & =\frac{1}{j \omega \varepsilon \rho} \frac{\partial^{2} \psi^{e}}{\partial \phi \partial z} & H_{\phi}^{e} & =-\frac{\partial \psi^{e}}{\partial \rho} \\
E_{z}^{e} & =\frac{1}{j \omega \varepsilon}\left(\frac{\partial^{2}}{\partial z^{2}}+k^{2}\right) \psi^{e} & H_{z}^{e} & =0
\end{aligned}
$$




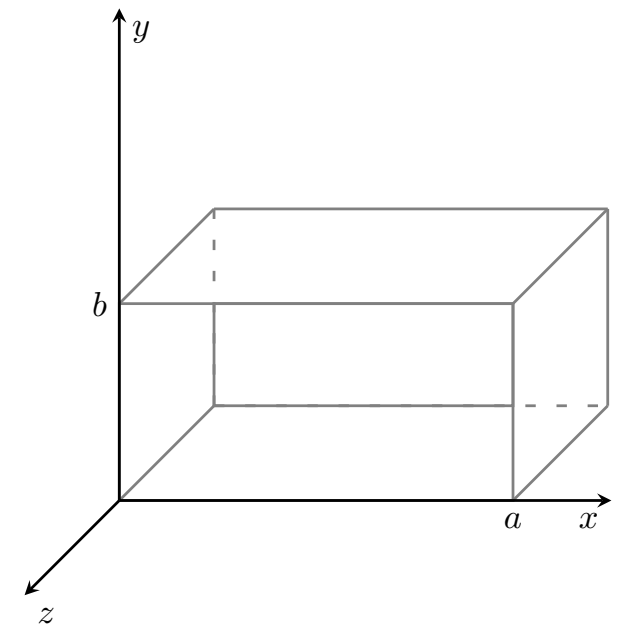

Figure A.1: Rectangular waveguide.

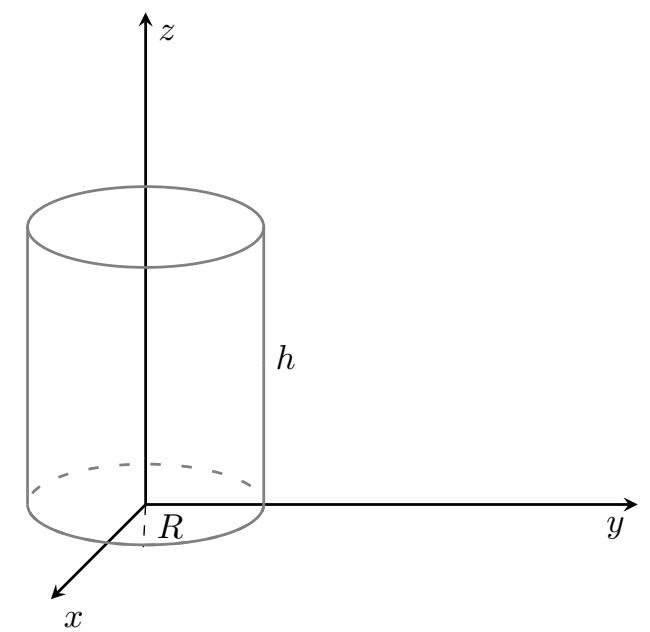

Figure A.2: Circular waveguide.

\section{A.2 Fields in Cavity and Hole regions}

Diagrams of the breakdown of the inhomogeneous cavity into regions for modeling are shown again in Figure A.3. The fields in the cavity and hole, or $C$ - and $H$-regions are equivalent, with the only difference being the radius $R$ of the outer conducting surface, so only the analysis for the cavity fields are presented. The wavenumbers and TE- and TM-wavefunctions are a more general version of A.3 for circular waveguide, allowing for inhomogeneous composition along with incoming and outgoing waves:

$$
\begin{gathered}
k_{i}=\omega \sqrt{\mu \varepsilon_{i}}, \quad k_{c_{\rho i}}=\sqrt{k_{i}^{2}-k_{c_{z}}^{2}} \\
\Psi_{c_{m}^{e}}^{h}=n_{c}\left[C_{c_{i}}^{h} J_{m}\left(k_{c_{\rho i}} \rho\right)+D_{c_{i}}^{h} Y_{m}\left(k_{c_{\rho i}} \rho\right)\right]\left\{\begin{array}{r}
\cos m \phi \\
-\sin m \phi
\end{array}\right\}\left[B_{c} e^{-j k_{c_{z} z} z}+A_{c} e^{+j k_{c_{z}} z}\right] \\
=n_{c} B_{c_{i}}^{h}(\rho)\left\{\begin{array}{c}
\cos m \phi \\
-\sin m \phi
\end{array}\right\} Z_{E}(z) \\
\Psi_{c_{m}^{e}}^{e}=n_{c}\left[C_{c_{i}}^{e} J_{m}\left(k_{c_{\rho i}} \rho\right)+D_{c_{i}}^{e} Y_{m}\left(k_{c_{\rho i}} \rho\right)\right]\left\{\begin{array}{c}
\sin m \phi \\
\cos m \phi
\end{array}\right\}\left[B_{c} e^{-j k_{c_{z} z} z}-A_{c} e^{+j k_{c_{z}} z}\right] \\
=n_{c} B_{c_{i}}^{e}(\rho)\left\{\begin{array}{c}
\sin m \phi \\
\cos m \phi
\end{array}\right\} Z_{H}(z)
\end{gathered}
$$

where $i$ is inner dielectric region 1,2, or 3 and $c$ designates a unique $C$-mode. The electric and magnetic symmetry walls at $\phi=0$ and $\pi$ define the $\phi$-functions in the braces, where the top function 


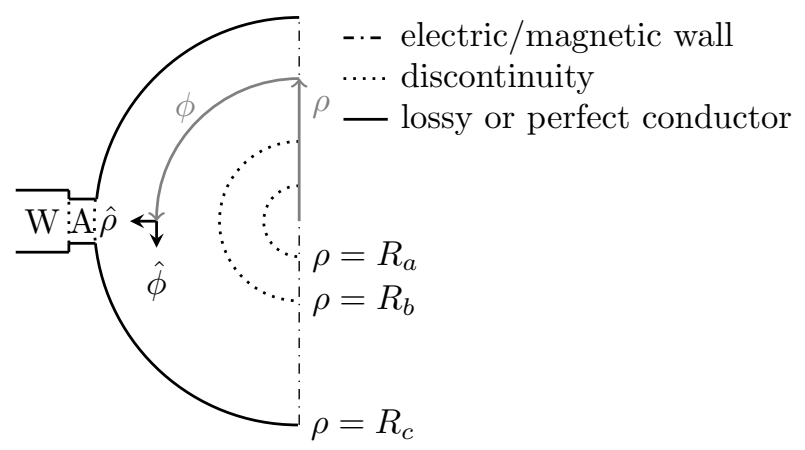

(a) Cross-section, $z=0$

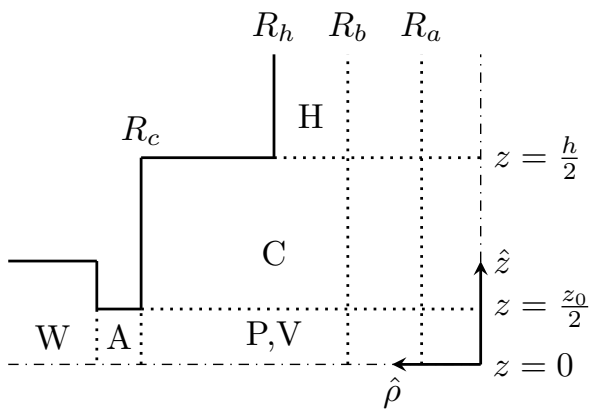

(b) Cross-section, $\phi=\frac{\pi}{2}$

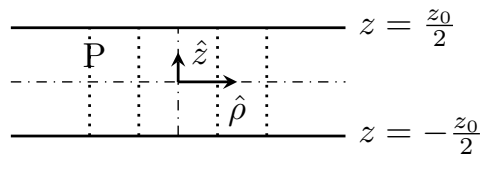

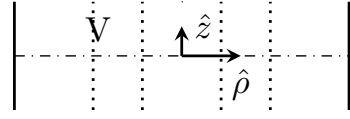

$$
\begin{aligned}
& \rho=R_{c}
\end{aligned}
$$

(c) $P$ - and $V$-intermediate regions for junction model. $P$ is radial waveguide with perfect conductors at $z=$ $\pm \frac{z_{0}}{2}$, while $V$ is inhomogeneous circular waveguide with perfect conductor at $\rho=R_{c}$.

Figure A.3: Diagram of regions and discontinuities for modeling.

is for the electric wall and the bottom one is for the magnetic wall. The $\phi$-indices $m$, which are also the order of the Bessel functions, are integers. Negative signs which appear arbitrary are to maintain consistent functions when derivatives are taken, which is useful later for making cancellations in the formulation of the inhomogeneous propagation constants. In region 1, or for an empty cavity, $D_{c_{i}}^{h, e}=0$ since $Y_{m}(\rho)$ is singular at $\rho=0$. Finally $n_{c}$ is a normalization factor that is added so that 
$\left\langle\vec{e}_{C}, \vec{h}_{C}\right\rangle_{\hat{z}}=1$. From the wavefunctions the fields can be found as

$$
\begin{aligned}
& \vec{E}_{c_{i}}^{h}=n_{c}\left[\hat{\rho} \frac{m}{\rho} B_{c_{i}}^{h}(\rho)\left\{\begin{array}{c}
\sin m \phi \\
\cos m \phi
\end{array}\right\}+\hat{\phi} k_{c_{\rho i}} B_{c_{i}}^{h^{\prime}}(\rho)\left\{\begin{array}{r}
\cos m \phi \\
-\sin m \phi
\end{array}\right\}\right] Z_{E}(z) \\
& \vec{H}_{c_{i}}^{h}=\frac{k_{c_{z}} n_{c}}{\omega \mu}\left[-\hat{\rho} k_{c_{\rho i}} B_{c_{i}}^{h \prime}(\rho)\left\{\begin{array}{r}
\cos m \phi \\
-\sin m \phi
\end{array}\right\}+\hat{\phi} \frac{m}{\rho} B_{c_{i}}^{h}(\rho)\left\{\begin{array}{c}
\sin m \phi \\
\cos m \phi
\end{array}\right\}\right] Z_{H}(z) \\
& +\hat{z} \frac{n_{c}\left(k_{i}^{2}-k_{c_{z}}^{2}\right)}{j \omega \mu} B_{c_{i}}^{h}(\rho)\left\{\begin{array}{r}
\cos m \phi \\
-\sin m \phi
\end{array}\right\} Z_{E}(z) \\
& \vec{E}_{c_{i}}^{e}=\frac{k_{c_{z}} n_{c}}{\omega \varepsilon_{i}}\left[-\hat{\rho} k_{c_{\rho i}} B_{c_{i}}^{e \prime}(\rho)\left\{\begin{array}{c}
\sin m \phi \\
\cos m \phi
\end{array}\right\}-\hat{\phi} \frac{m}{\rho} B_{c_{i}}^{e}(\rho)\left\{\begin{array}{r}
\cos m \phi \\
-\sin m \phi
\end{array}\right\}\right] Z_{E}(z) \\
& +\hat{z} \frac{n_{c}\left(k_{i}^{2}-k_{c_{z}}^{2}\right)}{j \omega \varepsilon_{i}} B_{c_{i}}^{e}(\rho)\left\{\begin{array}{c}
\sin m \phi \\
\cos m \phi
\end{array}\right\} Z_{H}(z) \\
& \vec{H}_{c_{i}}^{e}=n_{c}\left[\hat{\rho} \frac{m}{\rho} B_{c_{i}}^{e}(\rho)\left\{\begin{array}{r}
\cos m \phi \\
-\sin m \phi
\end{array}\right\}-\hat{\phi} k_{c_{\rho i}} B_{c_{i}}^{e \prime}(\rho)\left\{\begin{array}{c}
\sin m \phi \\
\cos m \phi
\end{array}\right\}\right] Z_{H}(z)
\end{aligned}
$$

For an inhomogeneous cylinder, the propagation constants $k_{c_{z}}$ and the Bessel coefficients $C_{c_{i}}^{h, e}, D_{c_{i}}^{h, e}$ in A.4a and A.4b are found numerically by satisfying the boundary conditions at the radial discontinuities. Specifically, at each inner radius $j$, which separates regions $a$ and $b$, the tangential electric and magnetic fields are equated. In the following, $J_{m(a, j)} \equiv J_{m}\left(k_{c_{\rho a}} \rho_{j}\right)$.

$$
\begin{array}{cc}
E_{\phi}: & k_{c_{\rho a} a}\left[C_{c_{a}}^{h} J_{m(a j)}^{\prime}+D_{c_{a}}^{h} Y_{m(a j)}^{\prime}\right]-\frac{k_{c_{z}} m}{\omega \varepsilon_{a} \rho_{j}}\left[C_{c_{a}}^{e} J_{m(a j)}+D_{c_{a}}^{e} Y_{m(a j)}\right] \\
=k_{c_{\rho b}}\left[C_{c_{b}}^{h} J_{m(b j)}^{\prime}+D_{c_{b}}^{h} Y_{m(b j)}^{\prime}\right]-\frac{k_{c_{z}} m}{\omega \varepsilon_{b} \rho_{j}}\left[C_{c_{b}}^{e} J_{m(b j)}+D_{c_{b}}^{e} Y_{m(b j)}\right] \\
E_{z}: \quad \frac{k_{a}^{2}-k_{c_{z}}^{2}}{\omega \varepsilon_{a}}\left[C_{c_{a}}^{e} J_{m(a j)}+D_{c_{a}}^{e} Y_{m(a j)}\right]=\frac{k_{b}^{2}-k_{c_{z}}^{2}}{\omega \varepsilon_{b}}\left[C_{c_{b}}^{e} J_{m(b j)}+D_{c_{b}}^{e} Y_{m(b j)}\right] \\
H_{\phi}: \quad \frac{k_{c_{z}} m}{\omega \mu \rho_{j}}\left[C_{c_{a}}^{h} J_{m(a j)}+D_{c_{a}}^{h} Y_{m(a j)}\right]-k_{c_{\rho a}}\left[C_{c_{a}}^{e} J_{m(a j)}^{\prime}+D_{c_{a}}^{e} Y_{m(a j)}^{\prime}\right] \\
\quad=\frac{k_{c_{z}} m}{\omega \mu \rho_{j}}\left[C_{c_{b}}^{h} J_{m(b j)}+D_{c_{b}}^{h} Y_{m(b j)}\right]-k_{\rho b}^{C}\left[C_{c_{b}}^{e} J_{m(b j)}^{\prime}+D_{c_{b}}^{e} Y_{m(b j)}^{\prime}\right] \\
H_{z}: \quad \frac{k_{a}^{2}-k_{c_{z}}^{2}}{\omega \mu}\left[C_{c_{a}}^{h} J_{m(a j)}+D_{c_{a}}^{h} Y_{m(a j)}\right]=\frac{k_{b}^{2}-k_{c_{z}}^{2}}{\omega \mu}\left[C_{c_{b}}^{h} J_{m(b j)}+D_{c_{b}}^{h} Y_{m(b j)}\right]
\end{array}
$$

At the outer radius $R_{c}$, a final boundary condition for the tangential electric fields of the third region (or single region for an empty cavity) is used, which takes into account a low-loss conductor, whose value is embedded in the surface impedance $Z_{s}$

$$
\vec{E}_{c_{T 3}}=Z_{s}(-\hat{\rho}) \times \vec{H}_{c_{T 3}}
$$


which gives

$$
\begin{gathered}
E_{c_{\phi 3}}=Z_{s} H_{c_{z 3}} \\
E_{c_{z 3}}=-Z_{s} H_{c_{\phi 3}} \\
E_{\phi}: \quad\left(k_{c_{\rho 3}} J_{m(3 c)}^{\prime}-Z_{s} \frac{k_{c_{\rho 3}}^{2}}{j \omega \mu} J_{m(3 c)}\right) C_{c_{3}}^{h}+\left(k_{c_{\rho 3}} Y_{m(3 c)}^{\prime}-Z_{s} \frac{k_{c_{\rho 3}}^{2}}{j \omega \mu} Y_{m(3 c)}\right) D_{c_{3}}^{h} \\
\quad-\frac{k_{c_{z}} m}{\omega \varepsilon_{3} \rho_{c}}\left(J_{m(3 c)} C_{c_{3}}^{e}+Y_{m(3 c)} D_{c_{3}}^{e}\right)=0 \\
E_{z}: \quad Z_{s} \frac{k_{c_{z}} m}{\omega \mu \rho_{c}}\left(J_{m(3 c)} C_{c_{3}}^{h}+Y_{m(3 c)} D_{c_{3}}^{h}\right) \\
+\left(\frac{k_{c_{\rho 3}}^{2}}{j \omega \varepsilon_{3}} J_{m(3 c)}-Z_{s} k_{c_{\rho 3}} J_{m(3 c)}^{\prime}\right) C_{c_{3}}^{e}+\left(\frac{k_{c_{\rho 3}}^{2}}{j \omega \varepsilon_{3}} Y_{m(3 c)}-Z_{s} k_{c_{\rho 3}} Y_{m(3 c)}^{\prime}\right) D_{c_{3}}^{e}
\end{gathered}
$$

These ten equations form a homogeneous linear system, and when combined into a matrix, the zero-valued determinant gives the values of $k_{c_{z}}$. With each known mode propagation constant, the corresponding nullspace of the system comprises the Bessel coefficients $C$ and $D$. Each mode is a hybrid mode, meaning it is composed of both TE- and TM-modes.

\section{A.3 Fields in $V$-region}

The $V$-region is similar to the $C$-region, except that the surface at radius $R_{c}$ is a perfect electric conductor with $Z_{s}=0$, and the $z$-component of the wavefunction is defined to satisfy the boundary conditions for electric and magnetic symmetry walls at $z=0$. As with the $C$-region, the extra negative signs and the extra $j$ factor in $\Psi_{v}$ are for maintaing a consistent formulation for finding $k_{v_{z}}, V_{v_{i}}$, and $W_{v_{i}}$. Each unique mode is signified by $v$. A normalization of $n_{v}$ is included but not necessary because these modes do not include scattering coefficients.

$$
k_{i}=\omega \sqrt{\mu \varepsilon_{i}}, \quad k_{v_{\rho i}}=\sqrt{k_{i}^{2}-k_{v_{z}}^{2}}
$$




$$
\begin{aligned}
\Psi_{v}^{h} & =n_{v}\left[V_{v_{i}}^{h} J_{m}\left(k_{v_{\rho i}} \rho\right)+W_{v_{i}}^{h} Y_{m}\left(k_{v_{\rho i}} \rho\right)\right]\left\{\begin{array}{r}
\cos m \phi \\
-\sin m \phi
\end{array}\right\}\left\{\begin{array}{c}
\sin k_{v_{z}} z \\
\cos k_{v_{z}} z
\end{array}\right\} A_{v} \\
& =n_{v} B_{v_{i}}^{h}(\rho)\left\{\begin{array}{r}
\cos m \phi \\
-\sin m \phi
\end{array}\right\}\left\{\begin{array}{c}
\sin k_{v_{z}} z \\
\cos k_{v_{z}} z
\end{array}\right\} A_{v} \\
\Psi_{v}^{e} & =j n_{v}\left[V_{v_{i}}^{e} J_{m}\left(k_{v_{\rho i}} \rho\right)+W_{v_{i}}^{e} Y_{m}\left(k_{v_{\rho i}} \rho\right)\right]\left\{\begin{array}{c}
\sin m \phi \\
\cos m \phi
\end{array}\right\}\left\{\begin{array}{c}
\cos k_{v_{z}} z \\
-\sin k_{v_{z}} z
\end{array}\right\} A_{v} \\
& =j n_{v} B_{v_{i}}^{e}(\rho)\left\{\begin{array}{c}
\sin m \phi \\
\cos m \phi
\end{array}\right\}\left\{\begin{array}{c}
\cos k_{v_{z} z} z \\
-\sin k_{v_{z}} z
\end{array}\right\} A_{v}
\end{aligned}
$$

$$
\begin{aligned}
& \vec{E}_{v_{i}}^{h}=n_{v}\left[\hat{\rho} \frac{m}{\rho} B_{v_{i}}^{h}(\rho)\left\{\begin{array}{c}
\sin m \phi \\
\cos m \phi
\end{array}\right\}+\hat{\phi} k_{v_{\rho i}} B_{v_{i}}^{h^{\prime}}(\rho)\left\{\begin{array}{r}
\cos m \phi \\
-\sin m \phi
\end{array}\right\}\right]\left\{\begin{array}{c}
\sin k_{v_{z} z} z \\
\cos k_{v_{z}} z
\end{array}\right\} A_{v} \\
& \vec{H}_{v_{i}}^{h}=\frac{k_{v_{z}} n_{v}}{j \omega \mu}\left[\hat{\rho} k_{v_{\rho i}} B_{v_{i}}^{h^{\prime}}(\rho)\left\{\begin{array}{r}
\cos m \phi \\
-\sin m \phi
\end{array}\right\}-\hat{\phi} \frac{m}{\rho} B_{v_{i}}^{h}(\rho)\left\{\begin{array}{c}
\sin m \phi \\
\cos m \phi
\end{array}\right\}\right]\left\{\begin{array}{r}
\cos k_{v_{z} z} z \\
-\sin k_{v_{z}} z
\end{array}\right\} A_{v} \\
& +\hat{z} \frac{n_{v}\left(k_{i}^{2}-k_{v_{z}}^{2}\right)}{j \omega \mu} B_{v_{i}}^{h}(\rho)\left\{\begin{array}{r}
\cos m \phi \\
-\sin m \phi
\end{array}\right\}\left\{\begin{array}{c}
\sin k_{v_{z} z} \\
\cos k_{v_{z}} z
\end{array}\right\} A_{v} \\
& \vec{E}_{v_{i}}^{e}=\frac{n_{v} k_{v_{z}}}{\omega \varepsilon_{i}}\left[-\hat{\rho} k_{v_{\rho i}} B_{v_{i}}^{e^{\prime}}(\rho)\left\{\begin{array}{c}
\sin m \phi \\
\cos m \phi
\end{array}\right\}-\hat{\phi} \frac{m}{\rho} B_{v_{i}}^{e}(\rho)\left\{\begin{array}{c}
\cos m \phi \\
-\sin m \phi
\end{array}\right\}\right]\left\{\begin{array}{c}
\sin k_{v_{z} z} z \\
\cos k_{v_{z}} z
\end{array}\right\} A_{v} \\
& +\hat{z} \frac{n_{v}\left(k_{i}^{2}-k_{v_{z}}^{2}\right)}{\omega \varepsilon_{i}} B_{v_{i}}^{e}(\rho)\left\{\begin{array}{r}
\sin m \phi \\
\cos m \phi
\end{array}\right\}\left\{\begin{array}{r}
\cos k_{v_{z} z} \\
-\sin k_{v_{z}} z
\end{array}\right\} A_{v} \\
& \vec{H}_{v_{i}}^{e}=j n_{v}\left[\hat{\rho} \frac{m}{\rho} B_{v_{i}}^{e}(\rho)\left\{\begin{array}{r}
\cos m \phi \\
-\sin m \phi
\end{array}\right\}-\hat{\phi} k_{v_{\rho i}} B_{v_{i}}^{e^{\prime}}(\rho)\left\{\begin{array}{c}
\sin m \phi \\
\cos m \phi
\end{array}\right\}\right]\left\{\begin{array}{r}
\cos k_{v_{z} z} z \\
-\sin k_{v_{z}} z
\end{array}\right\} A_{v}
\end{aligned}
$$

\section{A.4 Fields in $P$-region}

The wavefunctions are formulated for cylindrical parallel-plate waveguide, with perfect electric conductors at $z= \pm \frac{z_{0}}{2}$. At $z=0$, even integer values for $n$ satisfy the electric symmetry wall, while the odd integers are modes for the magnetic wall. Unique modes in each region $i$ are represented by $p$, which is simply a combination of $m$ and $n$. Unlike the hybrid modes in $C$ - and $V$-regions, the modes here are separable into TE and TM.

$$
k_{i}=\omega \sqrt{\mu \varepsilon_{i}}, \quad k_{p_{\rho i}}=\sqrt{k_{i}^{2}-\frac{n^{2} \pi^{2}}{z_{0}^{2}}}
$$




$$
\begin{aligned}
\Psi_{p_{i}}^{h} & =\left[J_{m}\left(k_{p_{\rho i}} \rho\right)+Q_{p_{i}}^{h} Y_{m}\left(k_{p_{\rho i}} \rho\right)\right]\left\{\begin{array}{r}
\cos m \phi \\
-\sin m \phi
\end{array}\right\} \sin \frac{n \pi\left(z+\frac{z_{0}}{2}\right)}{z_{0}} A_{p_{i}}^{h} \\
& =B_{p_{m i}}^{h}(\rho)\left\{\begin{array}{c}
\cos m \phi \\
-\sin m \phi
\end{array}\right\} \sin \frac{n \pi\left(z+\frac{z_{0}}{2}\right)}{z_{0}} A_{p_{i}}^{h} \\
\Psi_{p_{i}}^{e} & =\left[J_{m}\left(k_{p_{\rho i}} \rho\right)+Q_{p_{i}}^{e} Y_{m}\left(k_{p_{\rho i}} \rho\right)\right]\left\{\begin{array}{c}
\sin m \phi \\
\cos m \phi
\end{array}\right\} \cos \frac{n \pi\left(z+\frac{z_{0}}{2}\right)}{z_{0}} A_{p_{i}}^{e} \\
& =B_{p_{m i}}^{e}(\rho)\left\{\begin{array}{c}
\sin m \phi \\
\cos m \phi
\end{array}\right\} \cos \frac{n \pi\left(z+\frac{z_{0}}{2}\right)}{z_{0}} A_{p_{i}}^{e}
\end{aligned}
$$

The fields are

$$
\begin{aligned}
\vec{E}_{p_{i}}^{h}= & {\left[-\hat{\rho} \frac{m}{\rho} B_{p_{m i}}^{h}(\rho)\left\{\begin{array}{r}
-\sin m \phi \\
-\cos m \phi
\end{array}\right\}+\hat{\phi} k_{p_{\rho i}} B_{p_{m i}}^{h^{\prime}}(\rho)\left\{\begin{array}{r}
\cos m \phi \\
-\sin m \phi
\end{array}\right\}\right] \sin \frac{n \pi\left(z+\frac{z_{0}}{2}\right)}{z_{0}} A_{p_{i}}^{h} } \\
\vec{H}_{p_{i}}^{h}= & {\left[\hat{\rho} \frac{n \pi}{j \omega \mu z_{0}} k_{p_{\rho i}} B_{p_{m i}}^{h^{\prime}}(\rho)\left\{\begin{array}{r}
\cos m \phi \\
-\sin m \phi
\end{array}\right\}+\hat{\phi} \frac{n \pi}{j \omega \mu z_{0}} \frac{m}{\rho} B_{p_{m i}}^{h}(\rho)\left\{\begin{array}{l}
-\sin m \phi \\
-\cos m \phi
\end{array}\right\}\right] \cos \frac{n \pi\left(z+\frac{z_{0}}{2}\right)}{z_{0}} } \\
& +\hat{z} \frac{\left(k_{i}^{2}-\left(\frac{n \pi}{z_{0}}\right)^{2}\right)}{j \omega \mu} B_{p_{m i}}^{h}(\rho)\left\{\begin{array}{c}
\cos m \phi \\
-\sin m \phi
\end{array}\right\} \sin \frac{n \pi\left(z+\frac{z_{0}}{2}\right)}{z_{0}} A_{p_{i}}^{h} \\
\vec{E}_{p_{i}}^{e}= & {\left[-\hat{\rho} \frac{n \pi}{j \omega \varepsilon_{i} z_{0}} k_{p_{\rho i}} B_{p_{m i}}^{e^{\prime}}(\rho)\left\{\begin{array}{c}
\sin m \phi \\
-\cos m \phi
\end{array}\right\}-\hat{\phi} \frac{n \pi}{j \omega \varepsilon_{i} z_{0}} \frac{m}{\rho} B_{p_{m i}}^{e}(\rho)\left\{\begin{array}{c}
\cos m \phi \\
-\sin m \phi
\end{array}\right\}\right] \sin \frac{n \pi\left(z+\frac{z_{0}}{2}\right)}{z_{0}} } \\
& +\hat{z} \frac{\left(k_{i}-\left(\frac{n \pi}{z_{0}}\right)^{2}\right)}{j \omega \varepsilon} B_{p_{m i}}^{e}(\rho)\left\{\begin{array}{c}
\sin m \phi \\
\cos m \phi
\end{array}\right\} \cos \frac{n \pi\left(z+\frac{z_{0}}{2}\right)}{z_{0}} A_{p_{i}}^{e} \\
\vec{H}_{p_{i}}^{e}= & {\left[\hat{\rho} \frac{m}{\rho} B_{p_{m i}}^{e}(\rho)\left\{\begin{array}{c}
\cos m \phi \\
-\sin m \phi
\end{array}\right\}-\hat{\phi} k_{p_{\rho i}} B_{p_{m i}}^{e^{\prime}}(\rho)\left\{\begin{array}{c}
\sin m \phi \\
\cos m \phi
\end{array}\right]\right] \cos \frac{n \pi\left(z+\frac{z_{0}}{2}\right)}{z_{0}} A_{p_{i}}^{e} }
\end{aligned}
$$

If there are inner dielectrics, then the Bessel coefficients $A_{p i}^{h, e}$ and $Q_{p i}^{h, e}$ serve to satisfy the tangential field boundary conditions. As in 91, starting from $Q_{p 1}^{h, e}=0, A_{p 1}^{h, e}$ and $A_{p 2}^{h, e}$ are found in terms of $A_{p 3}^{h, e}$ and $Q_{p 2}^{h, e}$ and $Q_{p 3}^{h, e}$ are solved by doing mode-matching at each radius. In this analysis, all $Q_{p_{i}}^{h, e}$ are diagonal matrices. First, all of the $A_{p_{1}}$ coefficients, or $A_{1}$, are formulated from electric field 
boundary condition at $\rho=R_{a}$

$$
\begin{aligned}
\left\langle\vec{e}_{P_{1}}, \vec{h}_{P_{1}}\right\rangle_{\hat{\rho}} \mathbf{R}_{\mathbf{1} \mathbf{E}_{\mathbf{a}}}^{\mathbf{J}} A_{1} & =\left\langle\vec{e}_{P_{2}}, \vec{h}_{P_{1}}\right\rangle_{\hat{\rho}}\left(\mathbf{R}_{\mathbf{2} \mathbf{E}_{\mathbf{a}}}^{\mathbf{J}}+\mathbf{R}_{\mathbf{2} \mathbf{E}_{\mathbf{a}}}^{\mathbf{Y}} \mathbf{Q}_{\mathbf{2}}\right) A_{2} \\
\mathbf{P}_{\mathbf{1}} \mathbf{R}_{\mathbf{1} \mathbf{E}_{\mathbf{a}}}^{\mathbf{J}} A_{1} & =\mathbf{P}_{\mathbf{2 1}}^{\mathbf{T}}\left(\mathbf{R}_{\mathbf{2} \mathbf{E}_{\mathbf{a}}}^{\mathbf{J}}+\mathbf{R}_{\mathbf{2} \mathbf{E}_{\mathbf{a}}}^{\mathbf{Y}} \mathbf{Q}_{\mathbf{2}}\right) A_{2} \\
A_{1} & =\mathbf{R}_{\mathbf{1} \mathbf{E}_{\mathbf{a}}}^{\mathbf{J}} \mathbf{P}_{\mathbf{1}}^{-\mathbf{1}} \mathbf{P}_{\mathbf{2 1}}^{\mathbf{T}}\left(\mathbf{R}_{\mathbf{2} \mathbf{E}_{\mathbf{a}}}^{\mathbf{J}}+\mathbf{R}_{\mathbf{2} \mathbf{E}_{\mathbf{a}}}^{\mathbf{Y}} \mathbf{Q}_{\mathbf{2}}\right) A_{2}
\end{aligned}
$$

where $\mathbf{R}_{\mathbf{1}}^{\mathbf{J}} \mathbf{a}$ is a diagonal matrix of of the appropriate Bessel function of the first order (superscript $J$ ), for the electric field in region 1 at $\rho=R_{a}$ (subscripts $E, 1$, and $a$ ), e.g. $J_{m}^{\prime}\left(k_{p_{\rho 1}} R_{a}\right)$ for TEmodes. This relation is then substituted into the magnetic boundary condition so that a formulation for $\mathbf{Q}_{\mathbf{2}}$ is found

$$
\begin{aligned}
& \mathbf{P}_{\mathbf{2 1}} \mathbf{R}_{\mathbf{1} \mathbf{H}_{\mathbf{a}}}^{\mathbf{J}} A_{1}=\mathbf{P}_{\mathbf{2}}\left(\mathbf{R}_{\mathbf{2} \mathbf{H}_{\mathbf{a}}}^{\mathrm{J}}+\mathbf{R}_{\mathbf{2} \mathbf{H}_{\mathbf{a}}}^{\mathrm{Y}} \mathbf{Q}_{\mathbf{2}}\right) A_{2}
\end{aligned}
$$

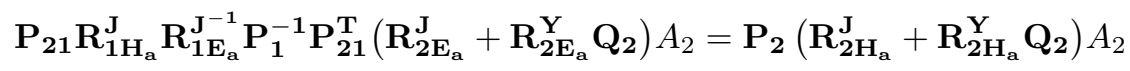

$$
\begin{aligned}
& \mathbf{K}_{\mathbf{2}} \mathbf{R}_{\mathbf{2}}^{\mathbf{J}} \mathbf{E}_{\mathbf{a}} A_{2}+\mathbf{K}_{\mathbf{2}} \mathbf{R}_{\mathbf{2} \mathbf{E}_{\mathbf{a}}}^{\mathbf{Y}} \mathbf{Q}_{\mathbf{2}} A_{2}=\mathbf{P}_{\mathbf{2}} \mathbf{R}_{\mathbf{2} \mathbf{H}_{\mathbf{a}}}^{\mathbf{J}} A_{2}+\mathbf{P}_{\mathbf{2}} \mathbf{R}_{\mathbf{2} \mathbf{H}_{\mathbf{a}}}^{\mathbf{Y}} \mathbf{Q}_{\mathbf{2}} A_{2} \\
& \mathbf{Q}_{2}=\left(\mathbf{K}_{2} \mathbf{R}_{2 \mathrm{E}_{\mathrm{a}}}^{\mathrm{Y}}-\mathbf{P}_{\mathbf{2}} \mathbf{R}_{2 \mathrm{H}_{\mathrm{a}}}^{\mathrm{Y}}\right)^{-1}\left(\mathbf{P}_{\mathbf{2}} \mathbf{R}_{2 \mathrm{H}_{\mathrm{a}}}^{\mathrm{J}}-\mathbf{K}_{\mathbf{2}} \mathbf{R}_{2 \mathrm{E}_{\mathrm{a}}}^{\mathrm{J}}\right)
\end{aligned}
$$

and $A_{2}$ is the remaining unknown. As in the electric field relation, $\mathbf{R}_{\mathbf{1}_{\mathbf{a}}}^{\mathbf{J}}$ are the Bessel functions of the magnetic field. This is repeated at $\rho=R_{b}$

$$
\begin{aligned}
A_{2} & =\left(\mathbf{R}_{\mathbf{2} \mathbf{E}_{\mathbf{b}}}^{\mathbf{J}}+\mathbf{R}_{\mathbf{2} \mathbf{E}_{\mathbf{b}}}^{\mathbf{Y}} \mathbf{Q}_{\mathbf{2}}\right)^{-\mathbf{1}} \mathbf{P}_{\mathbf{2}}^{-\mathbf{1}} \mathbf{P}_{\mathbf{3 2}}^{\mathbf{T}}\left(\mathbf{R}_{\mathbf{3} \mathbf{E}_{\mathbf{b}}}^{\mathbf{J}}+\mathbf{R}_{\mathbf{3} \mathbf{E}_{\mathbf{b}}}^{\mathbf{Y}} \mathbf{Q}_{\mathbf{3}}\right) A_{3} \\
& =\left(\mathbf{J}_{\mathbf{2 3}}+\mathbf{Y}_{\mathbf{2 3}} \mathbf{Q}_{\mathbf{3}}\right) A_{3}
\end{aligned}
$$


which gives $A_{2}$ in terms of $A_{3}$ and $\mathbf{Q}_{\mathbf{3}}$. With the magnetic boundary condition, $\mathbf{Q}_{\mathbf{3}}$ is found

$$
\begin{aligned}
& \mathbf{P}_{\mathbf{3 2}}\left(\mathbf{R}_{\mathbf{2} \mathbf{H}_{\mathrm{b}}}^{\mathrm{J}}+\mathbf{R}_{\mathbf{2} \mathbf{H}_{\mathrm{b}}}^{\mathrm{Y}} \mathbf{Q}_{\mathbf{2}}\right) A_{2}=\mathbf{P}_{\mathbf{3}}\left(\mathbf{R}_{\mathbf{3} \mathbf{H}_{\mathrm{b}}}^{\mathrm{J}}+\mathbf{R}_{\mathbf{3} \mathbf{H}_{\mathrm{b}}}^{\mathrm{Y}} \mathbf{Q}_{\mathbf{3}}\right) A_{3} \\
& \left(\mathbf{P}_{\mathbf{3 2}} \mathbf{R}_{\mathbf{2} \mathbf{H}_{\mathrm{b}}}^{\mathbf{J}}+\mathbf{P}_{\mathbf{3 2}} \mathbf{R}_{\mathbf{2} \mathbf{H}_{\mathrm{b}}}^{\mathbf{Y}} \mathbf{Q}_{\mathbf{2}}\right)\left(\mathbf{J}_{\mathbf{2 3}}+\mathbf{Y}_{\mathbf{2 3}} \mathbf{Q}_{\mathbf{3}}\right) A_{3}=\left(\mathbf{P}_{\mathbf{3}} \mathbf{R}_{\mathbf{3} \mathbf{H}_{\mathrm{b}}}^{\mathbf{J}}+\mathbf{P}_{\mathbf{3}} \mathbf{R}_{\mathbf{3} \mathbf{H}_{\mathrm{b}}}^{\mathbf{Y}} \mathbf{Q}_{\mathbf{3}}\right) A_{3} \\
& \mathbf{K}_{32}\left(\mathbf{J}_{23}+\mathbf{Y}_{23} \mathbf{Q}_{3}\right)=\mathbf{P}_{3} \mathbf{R}_{3 H_{\mathrm{b}}}^{\mathrm{J}}+\mathbf{P}_{3} \mathbf{R}_{3 \mathrm{H}_{\mathrm{b}}}^{\mathbf{Y}} \mathbf{Q}_{3} \\
& \mathbf{Q}_{3}=\left(\mathbf{K}_{32} \mathbf{Y}_{23}-\mathbf{P}_{3} \mathbf{R}_{3 \mathbf{H}_{\mathrm{b}}}^{\mathbf{Y}}\right)^{-1}\left(\mathbf{P}_{3} \mathbf{R}_{3 \mathbf{H}_{\mathrm{b}}}^{\mathbf{J}}-\mathbf{K}_{32} \mathbf{J}_{23}\right)
\end{aligned}
$$

and thus $A_{1}$ and $A_{2}$ are expressed in terms of $A_{3}$, which is henceforth written as $A_{P}$.

\section{A.5 Fields in rectangular waveguide regions}

As in the analysis of rectangular waveguide starting with A.2, the wavenumbers and wavefunctions in the aperture (and likewise the input and output WR-10 waveguide) are defined as

$$
\begin{gathered}
k=\omega \sqrt{\mu \varepsilon}, \quad k_{a_{z}}=\sqrt{\frac{m^{2} \pi^{2}}{a_{x}^{2}}+\frac{n^{2} \pi^{2}}{b_{y}^{2}}-k^{2}} \\
\Psi_{a_{m}}^{h}=h_{a} \cos \frac{m \pi x_{A}}{a_{x}} \cos \frac{n \pi y_{A}}{b_{y}}\left(B_{a_{m}}^{h} e^{-k_{a}^{h} z_{A}}+A_{a_{m}}^{h} e^{+k_{a}^{h} z_{A}}\right) \\
\Psi_{a_{m}^{e}}^{e}=e_{a} \sin \frac{m \pi x_{A}}{a_{x}} \sin \frac{n \pi y_{A}}{b_{y}}\left(B_{a_{m}}^{e} e^{-k_{a}^{e} z_{A}}-A_{a_{m}^{e}}^{e} e^{+k_{a}^{e} z_{A}}\right)
\end{gathered}
$$


where $m$ and $n$ are integers that are modal indices, which are combined in the unique mode identifier subscript $a$. Each mode is normalized by $h_{a}$ and $e_{a}$. The fields are found as

$$
\begin{aligned}
\vec{E}_{a}^{h}= & h_{a}\left[\hat{x}_{A} \frac{n \pi}{b_{y}} \cos \frac{m \pi x_{A}}{a_{x}} \sin \frac{n \pi y_{A}}{b_{y}}-\hat{y}_{A} \frac{m \pi}{a_{x}} \sin \frac{m \pi x_{A}}{a} \cos \frac{n \pi y_{A}}{b_{y}}\right]\left(B_{a_{m}^{e}}^{h} e^{-k_{a}^{h} z_{A}}+A_{a_{m}^{e}}^{h} e^{+k_{a} z_{A}}\right) \\
\vec{H}_{a}^{h}= & \frac{h_{a} k_{a}^{h}}{j \omega \mu}\left[\hat{x}_{A} \frac{m \pi}{a_{x}} \sin \frac{m \pi x_{A}}{a_{x}} \cos \frac{n \pi y_{A}}{b_{y}}+\hat{y}_{A} \frac{n \pi}{b_{y}} \cos \frac{m \pi x_{A}}{a_{x}} \sin \frac{n \pi y_{A}}{b_{y}}\right]\left(B_{a_{m}^{e}}^{h} e^{-k_{a}^{h} z_{A}}-A_{a_{m}^{e}}^{h} e^{+k_{a}^{h} z_{A}}\right) \\
& +\hat{z}_{A} \frac{h_{a}\left(k^{2}+k_{a}^{2}\right)}{j \omega \mu} \cos \frac{m \pi x_{A}}{a_{x}} \cos \frac{n \pi y_{A}}{b_{y}}\left(B_{a_{m}^{e}}^{h} e^{-k_{a}^{h} z_{A}}+A_{a_{m}^{e}}^{h} e^{+k_{a}^{h} z_{A}}\right) \\
\vec{E}_{a}^{e}= & \frac{e_{a} k_{a}^{e}}{j \omega \varepsilon}\left[-\hat{x}_{A} \frac{m \pi}{a_{x}} \cos \frac{m \pi x_{A}}{a_{x}} \sin \frac{n \pi y_{A}}{b_{y}}-\hat{y}_{A} \frac{n \pi}{b_{y}} \sin \frac{m \pi x_{A}}{a_{x}} \cos \frac{n \pi y_{A}}{b_{y}}\right]\left(B_{a_{m}^{e}}^{e} e^{-k_{a}^{e} z_{A}}+A_{a_{m}^{e}}^{e} e^{+k_{a}^{e} z_{A}}\right) \\
& \quad+\hat{z}_{A} \frac{e_{a}\left(k^{2}+k_{a}^{e^{2}}\right)}{j \omega \varepsilon} \sin \frac{m \pi x_{A}}{a_{x}} \sin \frac{n \pi y_{A}}{b_{y}}\left(B_{a_{m}^{e}}^{e} e^{-k_{a}^{e} z_{A}}-A_{a_{m}^{e}}^{e} e^{+k_{a}^{e} z_{A}}\right) \\
\vec{H}_{a}^{e}= & e_{a}\left[\hat{x}_{A} \frac{n \pi}{b_{y}} \sin \frac{m \pi x_{A}}{a_{x}} \cos \frac{n \pi y_{A}}{b_{y}}-\hat{y}_{A} \frac{m \pi}{a_{x}} \cos \frac{m \pi x_{A}}{a_{x}} \sin \frac{n \pi y_{A}}{b_{y}}\right]\left(B_{a_{m}^{e}}^{e} e^{-k_{a}^{e} z_{A}}-A_{a_{m}^{e}}^{e} e^{+k_{a}^{e} z_{A}}\right)
\end{aligned}
$$

\section{A.5.1 Rectangular-to-cylindrical coordinate transformation}

To apply the mode-matching method at the cavity-aperture boundary, there needs to be a common coordinate system in order to do the cross-product integrations. The diagram in Figure A.4 shows how the cylindrical and rectangular coordinate systems in each region relate to one another. So that an integration is not done on Bessel functions, which would be computationally intensive, the aperture is transformed into cylindrical coordinates, allowing for the integration to be done over a constant $\rho=R_{c}$. A consequence of this is that $\hat{\rho}$ is completely normal to the surface, while $\hat{z}_{A}$ is not, so the reaction matrices in 2.6 and $(2.7)$ are not common factors of the incoming and outgoing aperture waves. Instead, these waves are a function of $\phi$, over which the cross-product reactions are integrated. The aperture coordinates are expressed in terms of the cavity coordinates as

$$
\begin{aligned}
\hat{x}_{A}=\hat{z} & x_{A}=z \\
\hat{y}_{A}=\hat{\rho} \cos \phi-\hat{\phi} \sin \phi & y_{A}=\rho \cos \phi \\
\hat{z}_{A}=\hat{\rho} \sin \phi+\hat{\phi} \cos \phi & z_{A}=\rho \sin \phi
\end{aligned}
$$


and the transformed fields, $\hat{\rho}$-tangential, are

$$
\begin{aligned}
& \vec{E}_{a}^{h}=h_{a}\left[\hat{\phi} \frac{m \pi}{a_{x}} \sin \frac{m \pi z}{a} \sin \phi \cos \frac{n \pi \rho \cos \phi}{b_{y}}+\hat{z} \frac{n \pi}{b_{y}} \cos \frac{m \pi z}{a_{x}} \sin \frac{n \pi \rho \cos \phi}{b_{y}}\right] . \\
& \left(B_{a}^{h} e^{-k_{a}^{h} \rho \sin \phi}+A_{a}^{h} e^{+k_{a} \rho \sin \phi}\right) \\
& \vec{H}_{a}^{h}=\frac{h_{a} k_{a}^{h}}{j \omega \mu}\left[\hat{z} \frac{m \pi}{a_{x}} \sin \frac{m \pi z}{a_{x}} \cos \frac{n \pi \rho \cos \phi}{b_{y}}-\hat{\phi} \frac{n \pi}{b_{y}} \cos \frac{m \pi z}{a_{x}} \sin \phi \sin \frac{n \pi \rho \cos \phi}{b_{y}}\right] . \\
& \left(B_{a}^{h} e^{-k_{a}^{h} \rho \sin \phi}-A_{a_{m}^{e}}^{h} e^{+k_{a}^{h} \rho \sin \phi}\right) \\
& +\hat{\phi} \frac{h_{a}\left(k^{2}+k_{a}^{2}\right)}{j \omega \mu} \cos \frac{m \pi z}{a_{x}} \cos \phi \cos \frac{n \pi \rho \cos \phi}{b_{y}}\left(B_{a_{m}^{e}}^{h} e^{-k_{a}^{h} \rho \sin \phi}+A_{a_{m}^{e}}^{h} e^{+k_{a}^{h} \rho \sin \phi}\right) \\
& \vec{E}_{a}^{e}=\frac{e_{a} k_{a}^{e}}{j \omega \varepsilon}\left[\hat{\phi} \frac{n \pi}{b_{y}} \sin \frac{m \pi z}{a_{x}} \sin \phi \cos \frac{n \pi \rho \cos \phi}{b_{y}}-\hat{z} \frac{m \pi}{a_{x}} \cos \frac{m \pi z}{a_{x}} \sin \frac{n \pi \rho \cos \phi}{b_{y}}\right] . \\
& \left(B_{a_{m}^{e}}^{e} e^{-k_{a}^{e} \rho \sin \phi}+A_{a_{m}^{e}}^{e} e^{+k_{a}^{e} \rho \sin \phi}\right) \\
& +\hat{\phi} \frac{e_{a}\left(k^{2}+k_{a}^{e^{2}}\right)}{j \omega \varepsilon} \sin \frac{m \pi z}{a_{x}} \cos \phi \sin \frac{n \pi \rho \cos \phi}{b_{y}}\left(B_{a_{m}^{e}}^{e} e^{-k_{a}^{e} \rho \sin \phi}-A_{a_{m}^{e}}^{e} e^{+k_{a}^{e} \rho \sin \phi}\right) \\
& \vec{H}_{a}^{e}=e_{a}\left[\hat{z} \frac{n \pi}{b_{y}} \sin \frac{m \pi z}{a_{x}} \cos \frac{n \pi \rho \cos \phi}{b_{y}}+\hat{\phi} \frac{m \pi}{a_{x}} \cos \frac{m \pi z}{a_{x}} \sin \phi \sin \frac{n \pi \rho \cos \phi}{b_{y}}\right] \text {. } \\
& \left(B_{a_{m}^{e}}^{e} e^{-k_{a}^{e} \rho \sin \phi}-A_{a_{m}^{e}}^{e} e^{+k_{a}^{e} \rho \sin \phi}\right)
\end{aligned}
$$

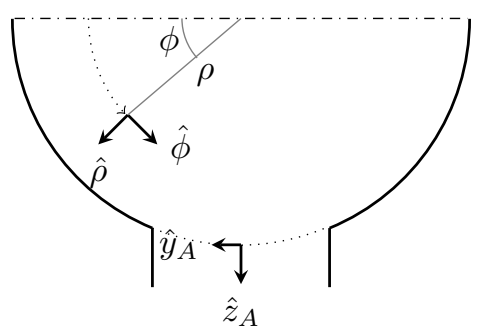

Figure A.4: Diagram of coordinate systems in cavity and aperture regions. 


\section{A.6 Full boundary conditions}

At $z=\frac{z_{0}}{2}$, enforcement of the boundary conditions produces

$$
\begin{aligned}
\hat{z} \times \vec{E}_{C} & =\hat{z} \times \vec{E}_{V} \\
\left\langle\vec{e}_{C}, \vec{h}_{V}\right\rangle\left(B_{C}+A_{C}\right) & =\left\langle\vec{e}_{V}, \vec{h}_{V}\right\rangle A_{V} \\
\mathbf{\Phi}_{\mathbf{C V}}\left(B_{C}+A_{C}\right) & =\mathbf{\Psi}_{\mathbf{V}} A^{V} \\
\hat{z} \times \vec{H}_{C} & =\hat{z} \times\left(\vec{H}_{V}+\vec{H}_{P}\right) \\
\left\langle\vec{e}_{C}, \vec{h}_{C}\right\rangle\left(B_{C}-A_{C}\right) & =\left\langle\vec{e}_{C}, \vec{h}_{V}\right\rangle A_{V}+\left\langle\vec{e}_{C}, \vec{h}_{P}\right\rangle A_{P} \\
\mathbf{\Psi}_{\mathbf{C}}\left(B_{C}-A_{C}\right) & =\mathbf{\Phi}_{\mathbf{C V}} A_{V}+\mathbf{\Phi}_{\mathbf{C P}} A_{P}
\end{aligned}
$$

At $\rho=R$, the boundary conditions are

$$
\begin{aligned}
& \hat{\rho} \times \vec{E}_{P}= \begin{cases}\hat{\rho} \times \vec{E}_{A} & \text { on } S_{A} \\
Z_{s}(-\hat{\rho}) \times\left(\vec{H}_{V}+\vec{H}_{P}\right) & \text { on } S_{P}-S_{A}\end{cases} \\
&\left\langle\vec{e}_{P}, \vec{h}_{P}\right\rangle A_{P}+Z_{s}\left\langle\left(\hat{\rho} \times \vec{h}_{P}\right), \vec{h}_{P}\right\rangle A_{P} \\
&+Z_{s}\left\langle\left(\hat{\rho} \times \vec{h}_{V}\right), \vec{h}_{P}\right\rangle A_{V}=\left\langle\vec{e}_{A}^{+}, \vec{h}_{P}\right\rangle B_{A}+\left\langle\vec{e}_{A}^{-}, \vec{h}_{P}\right\rangle A_{A} \\
&\left(\mathbf{\Psi}_{\mathbf{P}}-\mathbf{\Psi}_{\mathbf{P}}^{\mathbf{H}}\right) A_{P}+\mathbf{\Phi}_{\mathbf{V} \mathbf{P}}^{\mathbf{H}} A_{V}=\mathbf{\Phi}_{\mathbf{A} \mathbf{P}}^{+} B_{A}+\mathbf{\Phi}_{\mathbf{A} \mathbf{P}}^{-} A_{A} \\
& \hat{\rho} \times\left(\vec{H}_{P}+\vec{H}_{V}\right)=\hat{\rho} \times \vec{H}_{A} \\
&\left\langle\frac{1}{2}\left(\vec{e}_{A}^{+}+\vec{e}_{A}^{-}\right),\left(\vec{h}_{P} A_{P}+\vec{h}_{V} A_{V}\right)\right\rangle=\left\langle\frac{1}{2}\left(\vec{e}_{A}^{+}+\vec{e}_{A}^{-}\right),\left(\vec{h}_{A}^{+} B_{A}+\vec{h}_{A}^{-} A_{A}\right)\right\rangle \\
& \frac{1}{2}\left(\mathbf{\Phi}_{\mathbf{A P}}^{+\mathbf{T}}+\mathbf{\Phi}_{\mathbf{A P}}^{-\mathbf{T}}\right) A_{P}+\mathbf{\Phi}_{\mathbf{A V}} A_{V}=\mathbf{\Psi}_{\mathbf{A}}^{+} B_{A}+\mathbf{\Psi}_{\mathbf{A}}^{-} A_{A} \\
& \mathbf{K}_{\mathbf{A P}} A_{P}+\mathbf{\Phi}_{\mathbf{A V}} A_{V}=\mathbf{\Psi}_{\mathbf{A}}^{+} B_{A}+\mathbf{\Psi}_{\mathbf{A}}^{-} A_{A}
\end{aligned}
$$

where the choice of the weighting function $\frac{1}{2}\left(\vec{e}_{A}^{+}+\vec{e}_{A}^{-}\right)$in A.34b is from 59 . It is a combination of the outgoing and incoming waves, which are respectively represented by the superscripts ${ }^{+}$and $^{-}$, indicating their direction relative to $\hat{z}_{A}$. 
Solving for $A_{V}$ and $A_{P}$ in A.33a

$$
\begin{aligned}
A_{V} & =\mathbf{\Psi}_{\mathbf{V}}^{-\mathbf{1}} \mathbf{\Phi}_{\mathbf{C V}}\left(B_{C}+A_{C}\right) \\
& =\mathbf{M}_{\mathbf{V C}}\left(B_{C}+A_{C}\right)
\end{aligned}
$$

This is placed into A.34a so $A_{P}$ can be found

$$
\begin{aligned}
A_{P} & =\left(\mathbf{\Psi}_{\mathbf{P}}+\mathbf{\Psi}_{\mathbf{P}}^{\mathbf{H}}\right)^{-\mathbf{1}}\left[\mathbf{\Phi}_{\mathbf{A} \mathbf{P}}^{+} B_{A}+\mathbf{\Phi}_{\mathbf{A} \mathbf{P}}^{-} A_{A}-\mathbf{\Phi}_{\mathbf{V} \mathbf{P}}^{\mathbf{H}} \mathbf{M}_{\mathbf{V C}}\left(B_{C}+A_{C}\right)\right] \\
& =\mathbf{M}_{\mathbf{P} \mathbf{A}}^{+} B_{A}+\mathbf{M}_{\mathbf{P} \mathbf{R}}^{-} A_{A}-\mathbf{M}_{\mathbf{P V}} \mathbf{M}_{\mathbf{V C}}\left(B_{C}+A_{C}\right)
\end{aligned}
$$

Both of these can be substituted into the magnetic boundary conditions, starting with A.33b

$$
\begin{aligned}
\mathbf{\Psi}_{\mathbf{C}}\left(B_{C}-A_{C}\right)+\left(\mathbf{\Phi}_{\mathbf{C P}} \mathbf{M}_{\mathbf{P V}}-\mathbf{\Phi}_{\mathbf{C V}}\right) \mathbf{M}_{\mathbf{V C}}\left(B_{C}+A_{C}\right) & =\mathbf{\Phi}_{\mathbf{C P}} \mathbf{M}_{\mathbf{P A}}^{+} B_{A}+\mathbf{\Phi}_{\mathbf{C P}} \mathbf{M}_{\mathbf{P A}}^{-} A_{A} \\
\mathbf{H}_{\mathbf{B}_{\mathbf{C}}}^{\mathbf{z}} B_{C}+\mathbf{H}_{\mathbf{A}_{\mathbf{C}}}^{\mathbf{z}} A_{C} & =\mathbf{H}_{\mathbf{B}_{\mathbf{A}}}^{\mathbf{z}} B_{A}+\mathbf{H}_{\mathbf{A}_{\mathbf{A}}}^{\mathbf{z}} A_{A}
\end{aligned}
$$

and then A.34b

$$
\begin{aligned}
\left(\mathbf{\Phi}_{\mathbf{A V}} \mathbf{M}_{\mathbf{V C}}-\mathbf{K}_{\mathbf{A P}} \mathbf{M}_{\mathbf{P V}} \mathbf{M}_{\mathbf{V C}}\right) & \left(B_{C}+A_{C}\right) \\
= & \left(\mathbf{\Psi}_{\mathbf{A}}^{+}-\mathbf{K}_{\mathbf{A P}} \mathbf{M}_{\mathbf{P A}}^{+}\right) B_{A}+\left(\mathbf{\Psi}_{\mathbf{A}}^{-}-\mathbf{K}_{\mathbf{A P}} \mathbf{M}_{\mathbf{P A}}^{-}\right) A_{A} \\
\mathbf{H}_{\mathbf{B}_{\mathbf{C}}}^{\rho} B_{C}+\mathbf{H}_{\mathbf{A}_{\mathbf{C}}}^{\rho} A_{C} & =\mathbf{H}_{\mathbf{B}_{\mathbf{A}}}^{\rho} B_{A}+\mathbf{H}_{\mathbf{A}_{\mathbf{A}}}^{\rho} A_{A}
\end{aligned}
$$

Designating the aperture and its wave coefficients $A_{A}$ and $B_{A}$ as port 1, and the the circular region $C$ and $A_{C}$ and $B_{C}$ as port 2, the $S$-parameters of the junction can be found.

\section{A.6.1 Analytical Integrations}

Most of the integrations for the inner products are combinations of sine and cosine functions. For integrals over $\rho$, the expressions in papers by Kajfez and Manring were used 92, 93. 


\section{A.6.2 Small-angle approximation}

As the aperture width is much smaller than the cavity circumference, a small-angle approximation can be used for the surface integrals of the cross-product at $\rho=R_{c}$ discontinuity. Specifically, for an aperture that is centered at $\phi=\frac{\pi}{2}$, the integral over $\phi$, then a change of variable, is

$$
\int_{\frac{\pi}{2}-\theta}^{\frac{\pi}{2}+\theta} f(\phi) d \phi=\int_{-\theta}^{+\theta} f\left(\psi+\frac{\pi}{2}\right) d \psi
$$

For a small $\theta$, the coordinate-transformed aperture fields can then be approximated with the following

$$
\begin{array}{rrr}
\cos \phi & =\cos \left(\psi+\frac{\pi}{2}\right) & \sin \phi=\sin \left(\psi+\frac{\pi}{2}\right) \\
& =-\sin \psi & =\cos \psi \\
\approx-\psi & \approx 1
\end{array}
$$

\section{A.7 Cascading $S$-matrices}

A waveguide system frequently consists of multiple discontinuities. A diagram for combining two $S$-matrices that are separated by a constant waveguide section of electrical length $\ell$ is shown in Figure A.5

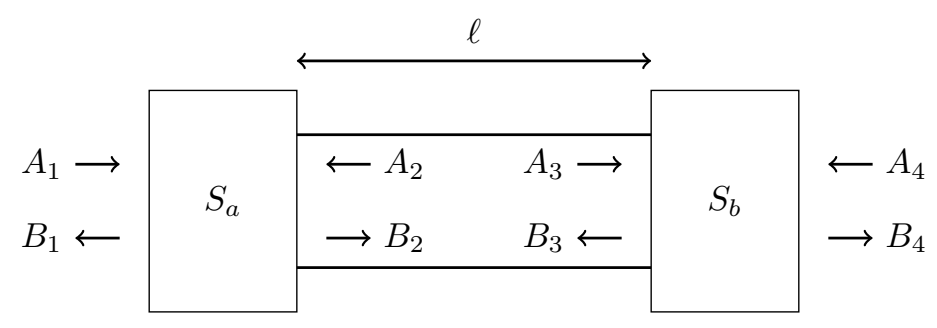

Figure A.5: Combination of two cascaded S-matrices.

In the end, what is desired is a final $S$-matrix $S^{\prime}$ with wave coefficients $A_{1}, B_{1}, A_{4}$, and $B_{4}$. First, it can be seen that $A_{2}$ is simply $B_{3}$ with a phase shift, and likewise for $A_{3}$ with regards to 
$B_{2}$. They can be defined as

$$
A_{2}=e^{-j k_{n} \ell} B_{3}=\mathbf{T} B_{3}
$$

where $k_{n}$ are the modal propagation constants in the center waveguide section and $\mathbf{T}$ is a square diagonal matrix. These are substituted into the relations between the outgoing and incoming waves

$$
\begin{aligned}
& B_{1}=\mathbf{S}_{\mathbf{1 1}} A_{1}+\mathbf{S}_{\mathbf{1 2}} \mathbf{T} B_{3} \\
& B_{2}=\mathbf{S}_{\mathbf{2 1}} A_{1}+\mathbf{S}_{\mathbf{2 2}} \mathbf{T} B_{3} \\
& B_{3}=\mathbf{S}_{\mathbf{3 3}} \mathbf{T} B_{2}+\mathbf{S}_{\mathbf{3 4}} A_{4} \\
& B_{4}=\mathbf{S}_{\mathbf{4 3}} \mathbf{T} B_{2}+\mathbf{S}_{\mathbf{4 4}} A_{4}
\end{aligned}
$$

Then, A.41c is substituted into A.41b, and $B_{2}$ is found, then substituted back into A.41c

$$
\begin{aligned}
& B_{2}=\mathbf{M}^{-1}\left(\mathbf{S}_{\mathbf{2 1}} A_{1}+\mathbf{S}_{\mathbf{2 2}} \mathbf{T} \mathbf{S}_{\mathbf{3 4}} A_{4}\right) \\
& B_{3}=\mathbf{S}_{\mathbf{3 3}} \mathbf{T} \mathbf{M}^{-1}\left(\mathbf{S}_{\mathbf{2 1}} A_{1}+\mathbf{S}_{\mathbf{2 2}} \mathbf{T} \mathbf{S}_{\mathbf{3 4}} A_{4}\right)+\mathbf{S}_{\mathbf{3 4}} A_{4} \\
& M=\left(\mathbf{I}-\mathbf{S}_{\mathbf{2 2}} \mathbf{T} \mathbf{S}_{\mathbf{3 3}} \mathbf{T}\right)
\end{aligned}
$$

When these relations replace $B_{2}$ and $B_{3}$ in A.41a and A.41d, only the desired wave coefficients are left, and the $S$-parameters are found as

$$
\begin{aligned}
& \mathbf{S}_{11}^{\prime}=\mathbf{S}_{11}+\mathrm{S}_{12} \mathrm{TS}_{33} \mathrm{TM}^{-1} \mathbf{S}_{21} \\
& \mathbf{S}_{14}^{\prime}=\mathbf{S}_{12} \mathrm{TS}_{33} \mathrm{TM}^{-1} \mathbf{S}_{22} \mathbf{T S}_{34} \\
& \mathbf{S}_{41}^{\prime}=\mathbf{S}_{43} \mathrm{TM}^{-1} \mathbf{S}_{21} \\
& \mathbf{S}_{41}^{\prime}=\mathbf{S}_{43} \mathrm{TM}^{-1} \mathbf{S}_{22} \mathrm{TS}_{34}
\end{aligned}
$$




\section{A.8 Symmetrical $S$-matrix}

The $S$-matrix found from analysis of the regions in Figure A.3 is two-port, but the final structure is a symmetrical four-port. The following shows how the four-port is obtained from the two-port. Credit must be given to the helpful derivation provided by Sabbagh 94 .

First, the technique will be demonstrated with deriving a symmetrical two-port from a one-port. To start, an arbitrary two-port is shown in Figure A.6a at each port is is presumed that a modematching analysis has occurred and the interior coefficients are not shown. If the two-port is known to be symmetric, then mode-matching only needs to be done on one-port, and the full two-port GSM can be derived from it. This is accomplished by placing electric and magnetic walls at the line of symmetry. With an electrical wall, a voltage minimum will occur in the center, so the waves at ports 1 and 2 will be out of phase. In other words, incoming waves of $\frac{A_{1}}{2}$ at port 1 and $-\frac{A_{1}}{2}$ at port

2 will satisfy this configuration, and likewise $\frac{A_{2}}{2}$ at port 2 and $-\frac{A_{2}}{2}$ at port 1 . For the magnetic wall, a voltage maximum occurs, and the waves must be in phase. Then if we set

$$
B_{1}=B_{1}^{e}+B_{1}^{m}
$$

we can substitute in for $B_{1}^{e, m}$ simple relations that include the incoming waves $A_{1,2}$ and the $S^{e, m_{-}}$ matrices

$$
\begin{aligned}
B_{1} & =\frac{1}{2} S^{e}\left(A_{1}-A_{2}\right)+\frac{1}{2} S^{m}\left(A_{1}+A_{2}\right) \\
& =\frac{1}{2}\left(S^{m}+S^{e}\right) A_{1}+\frac{1}{2}\left(S^{m}-S^{e}\right) A_{2} \\
& \equiv S_{11} A_{1}+S_{12} A_{2}
\end{aligned}
$$

and since the structure is symmetric, $S_{21}=S_{12}$, although the same analysis can be used with $B_{2}$.

A four-port can be analyzed in terms of a two-port with two lines of symmetry. With regards to structure of interest in Figure A.3b, the combination of electric and magnetic walls and the resulting waves is shown in Figure A.7 port $W$ in the former diagram corresponds to port 1, and likewise port $H$ with port 3 . 


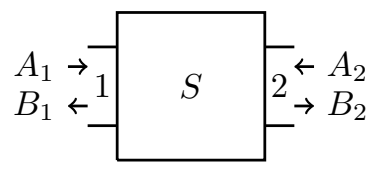

(a) Two-port $S$-matrix.

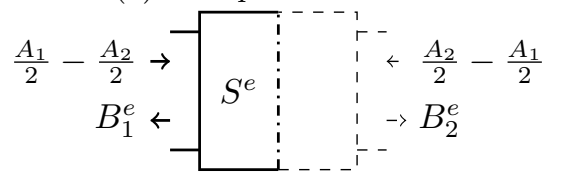

(b) Electric wall.

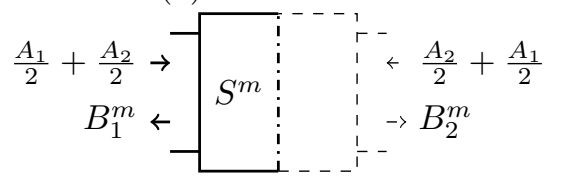

(c) Magnetic wall.

Figure A.6: Analysis of symmetrical 2-port from 1-port.
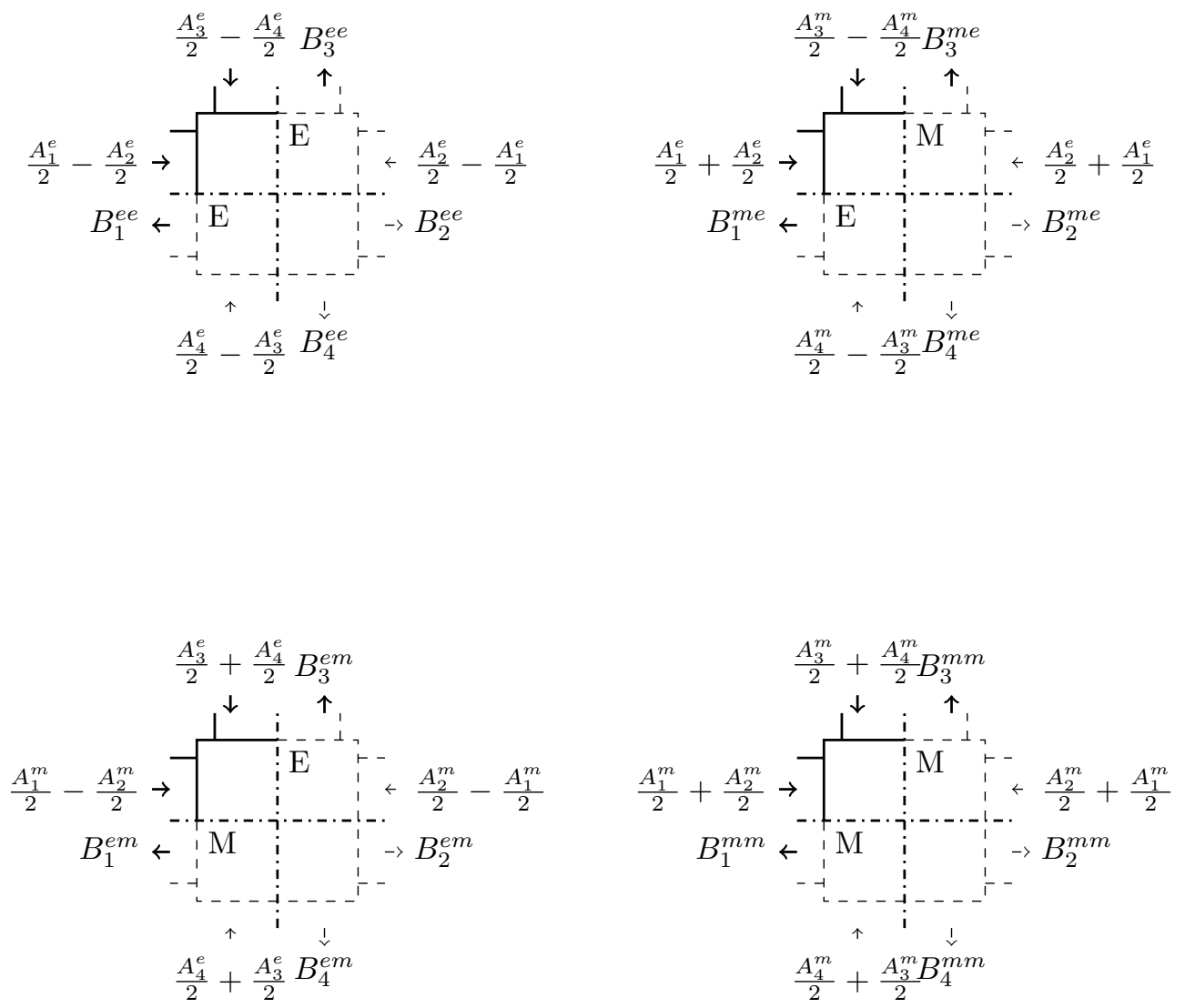

Figure A.7: Analysis of symmetrical 4-port from 2-port. 
Appendix B

\section{Drawings}




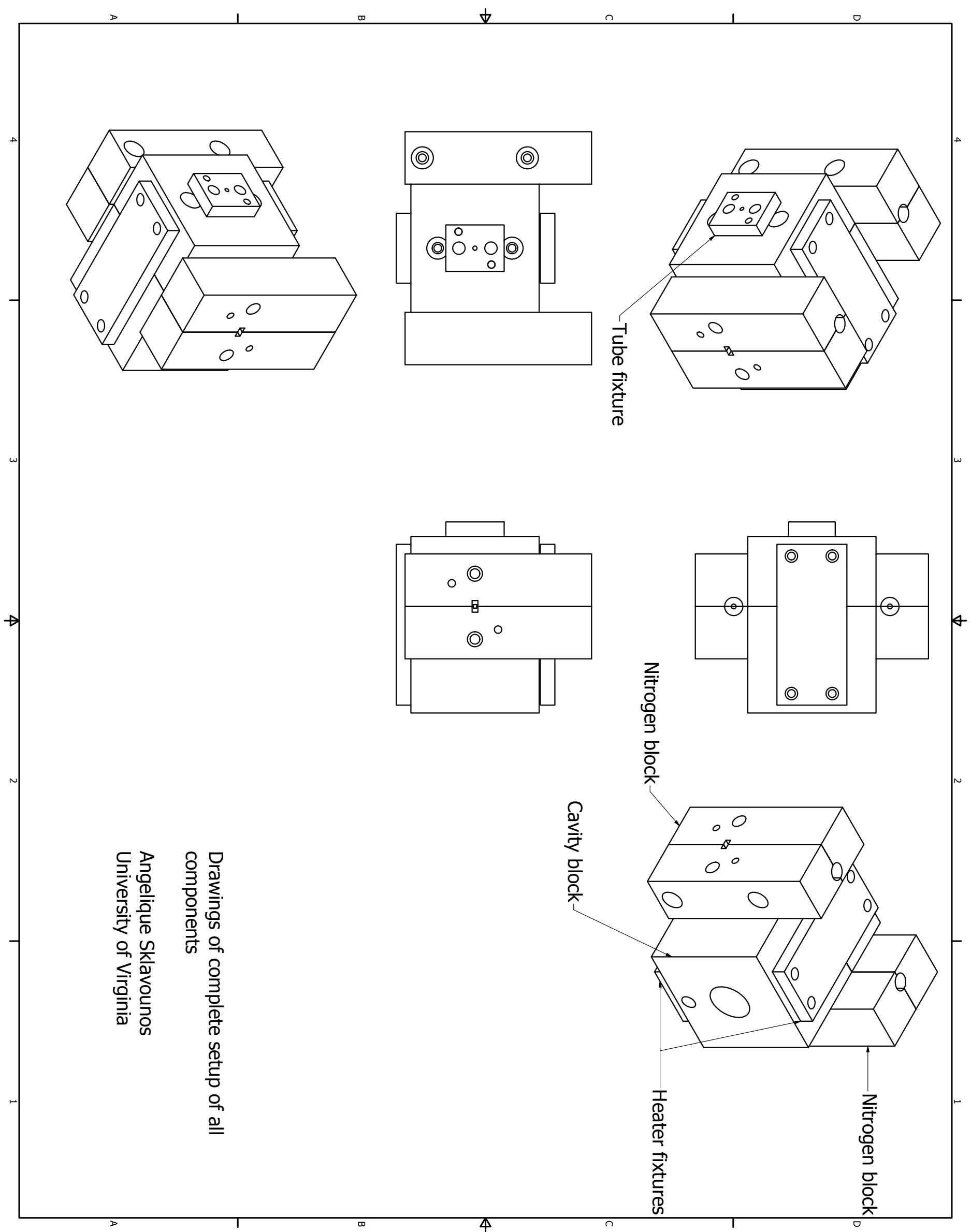




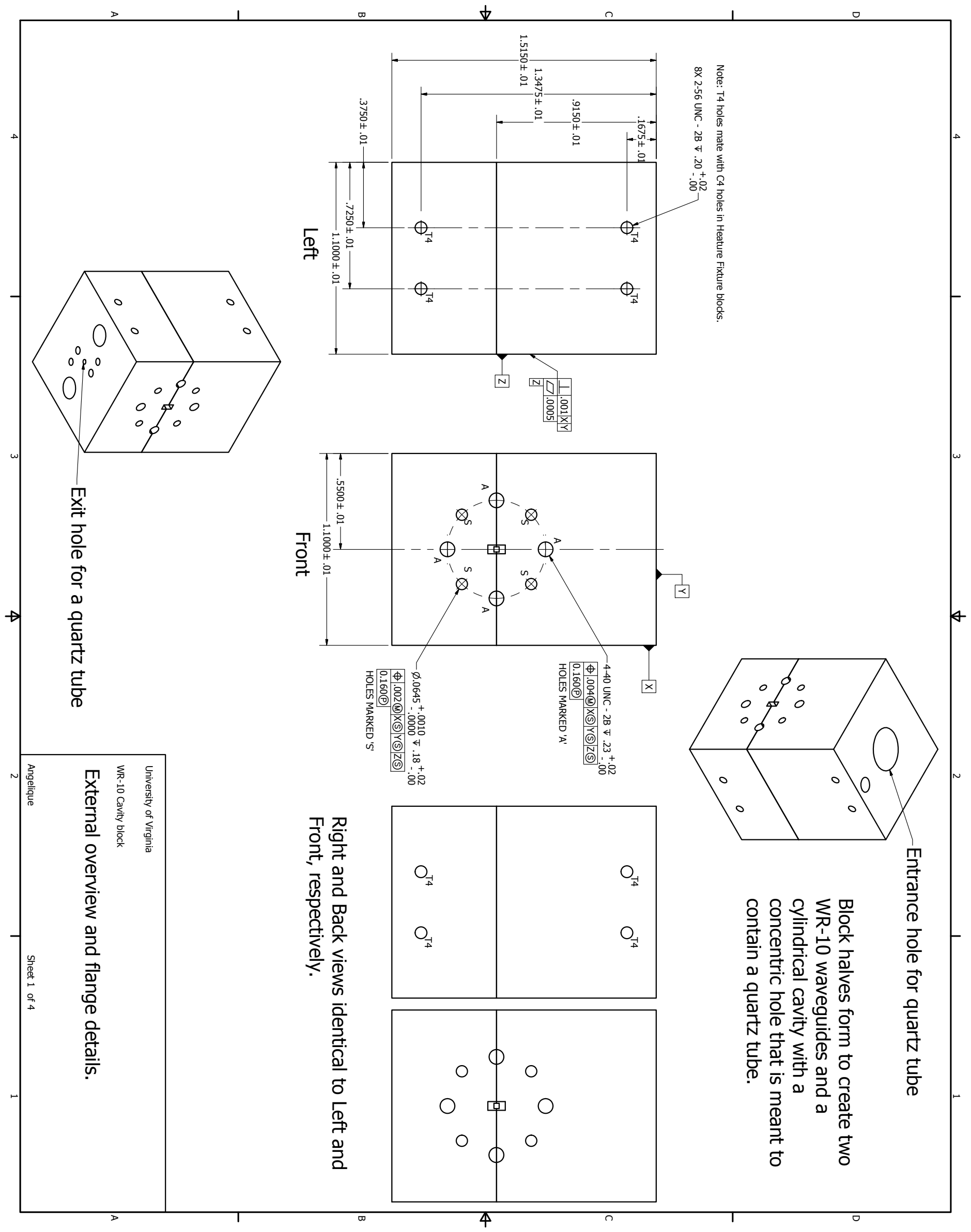




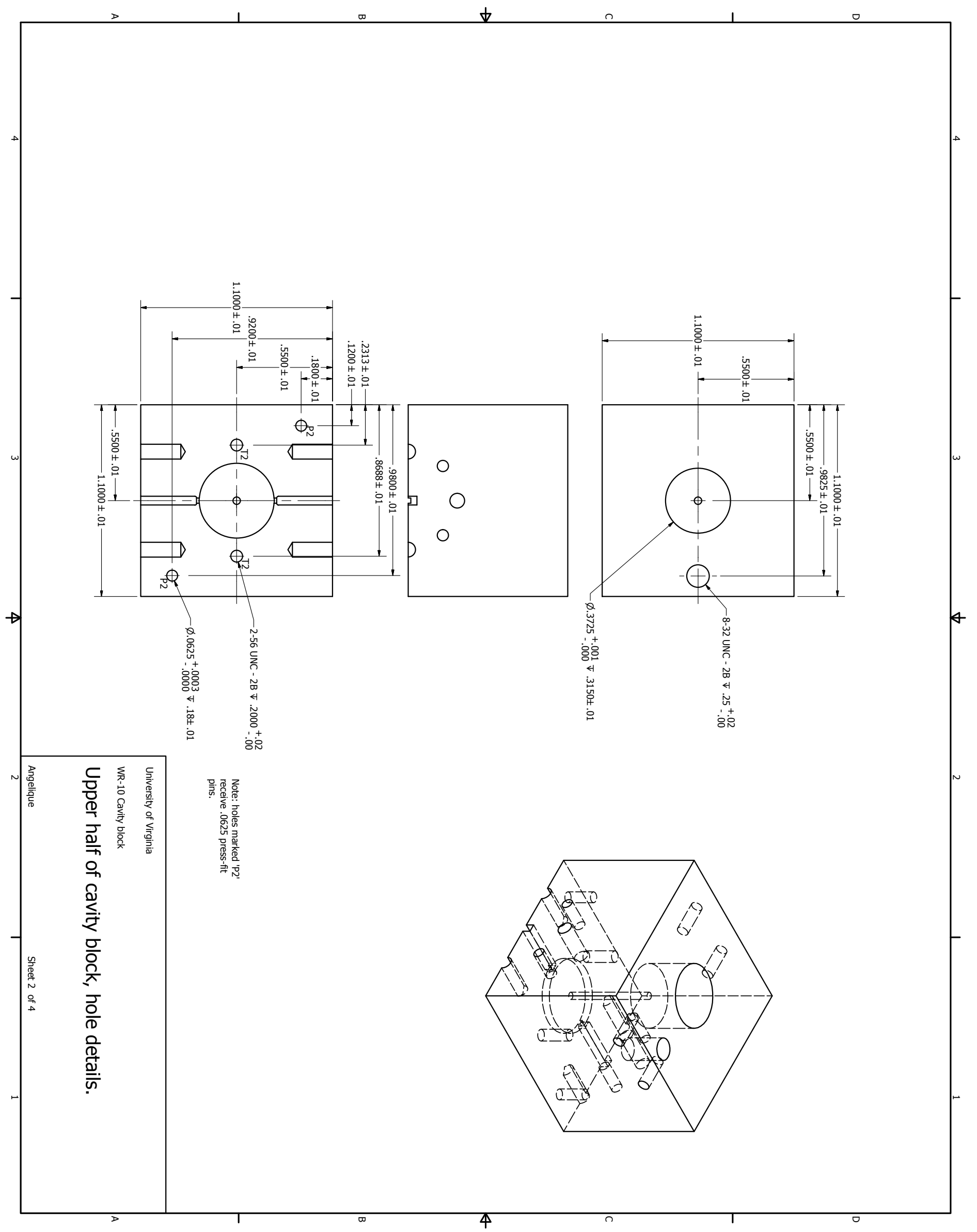




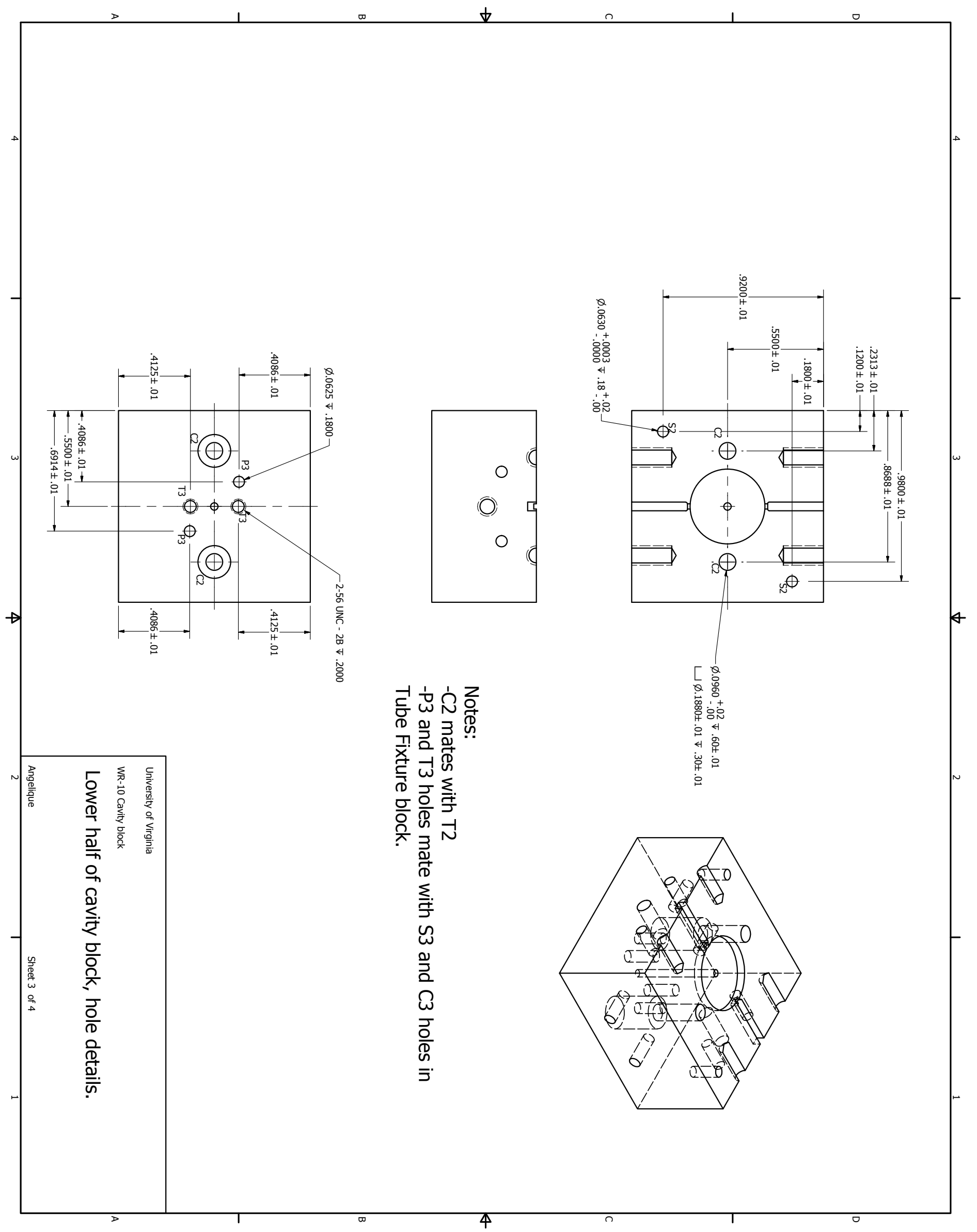




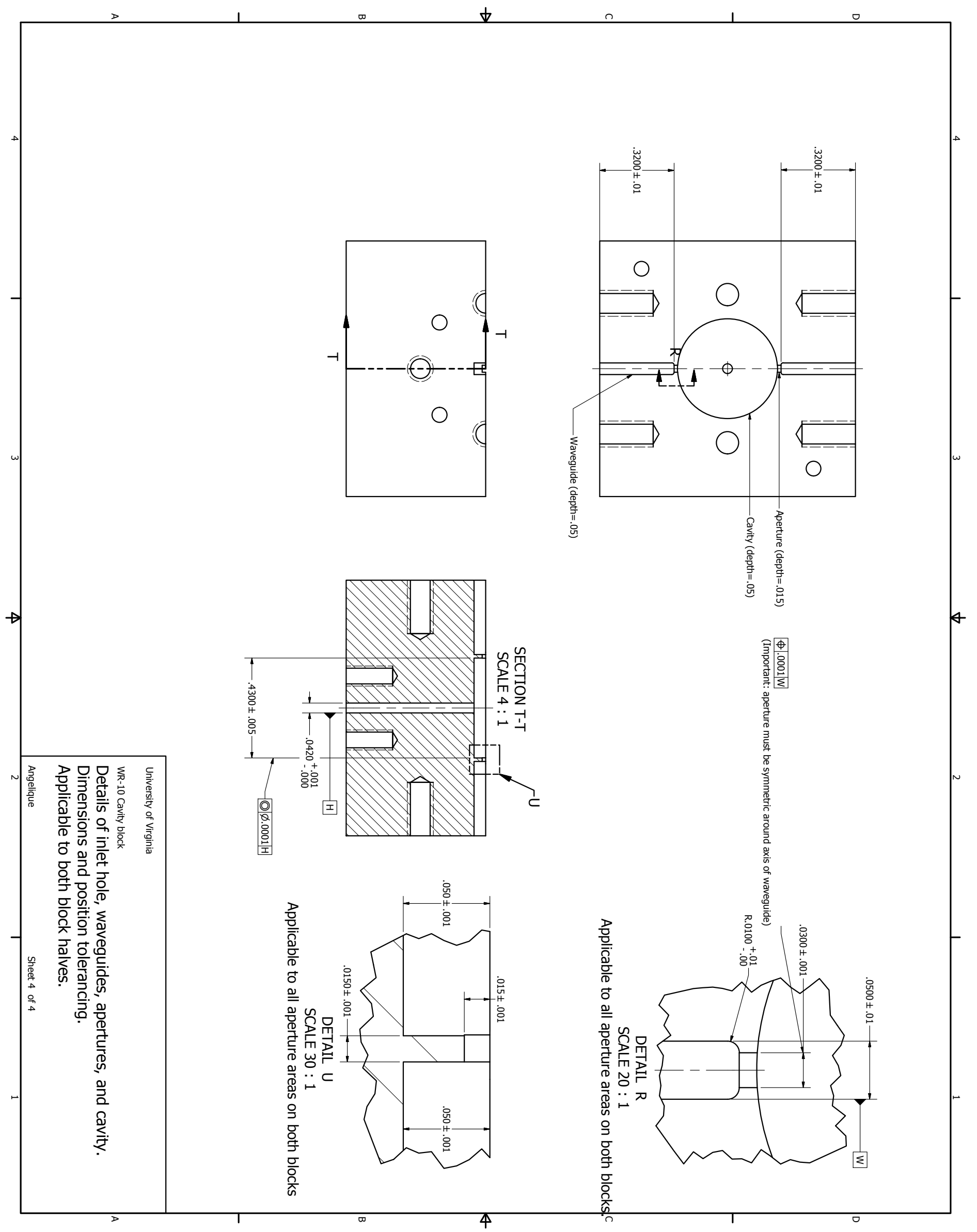




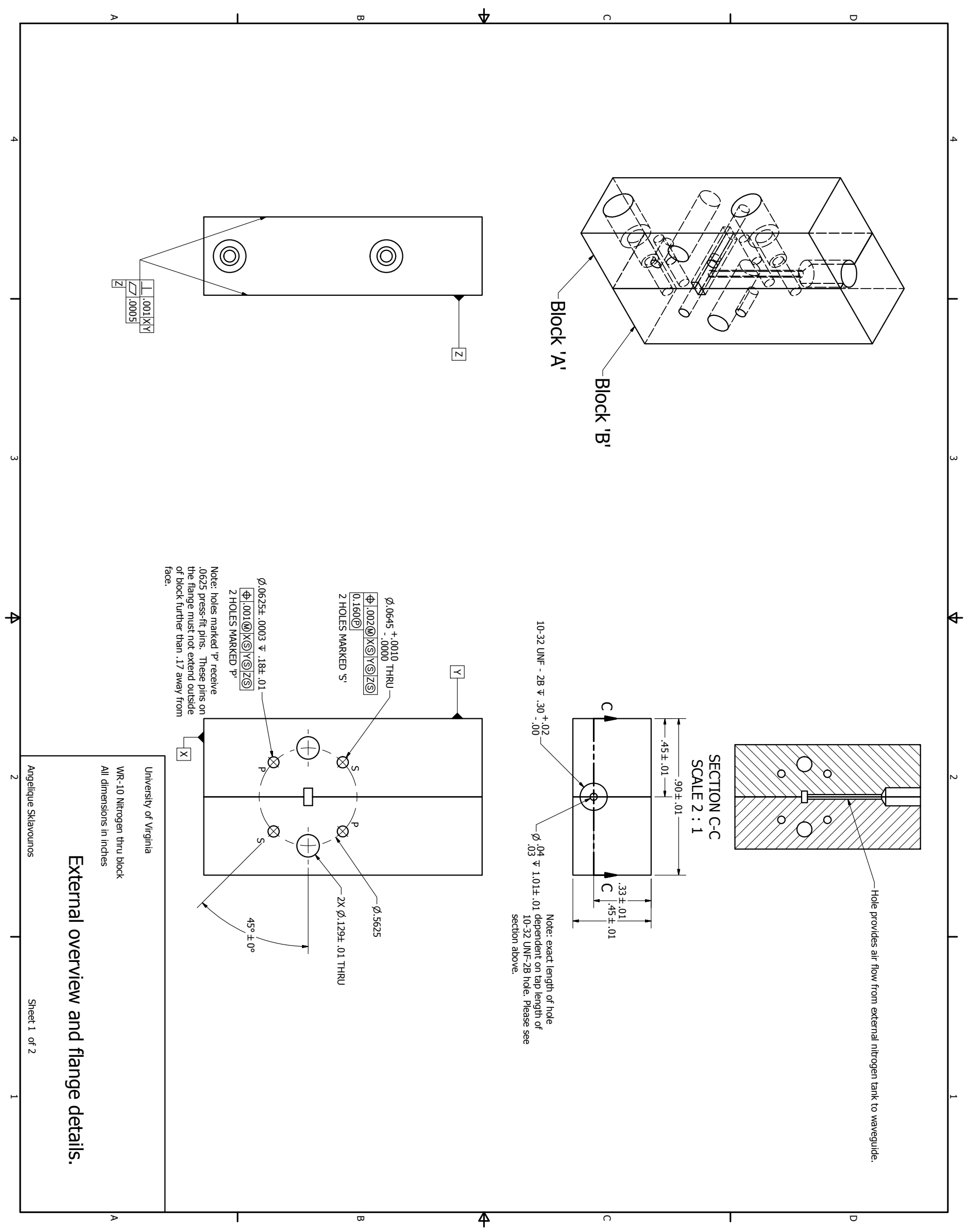




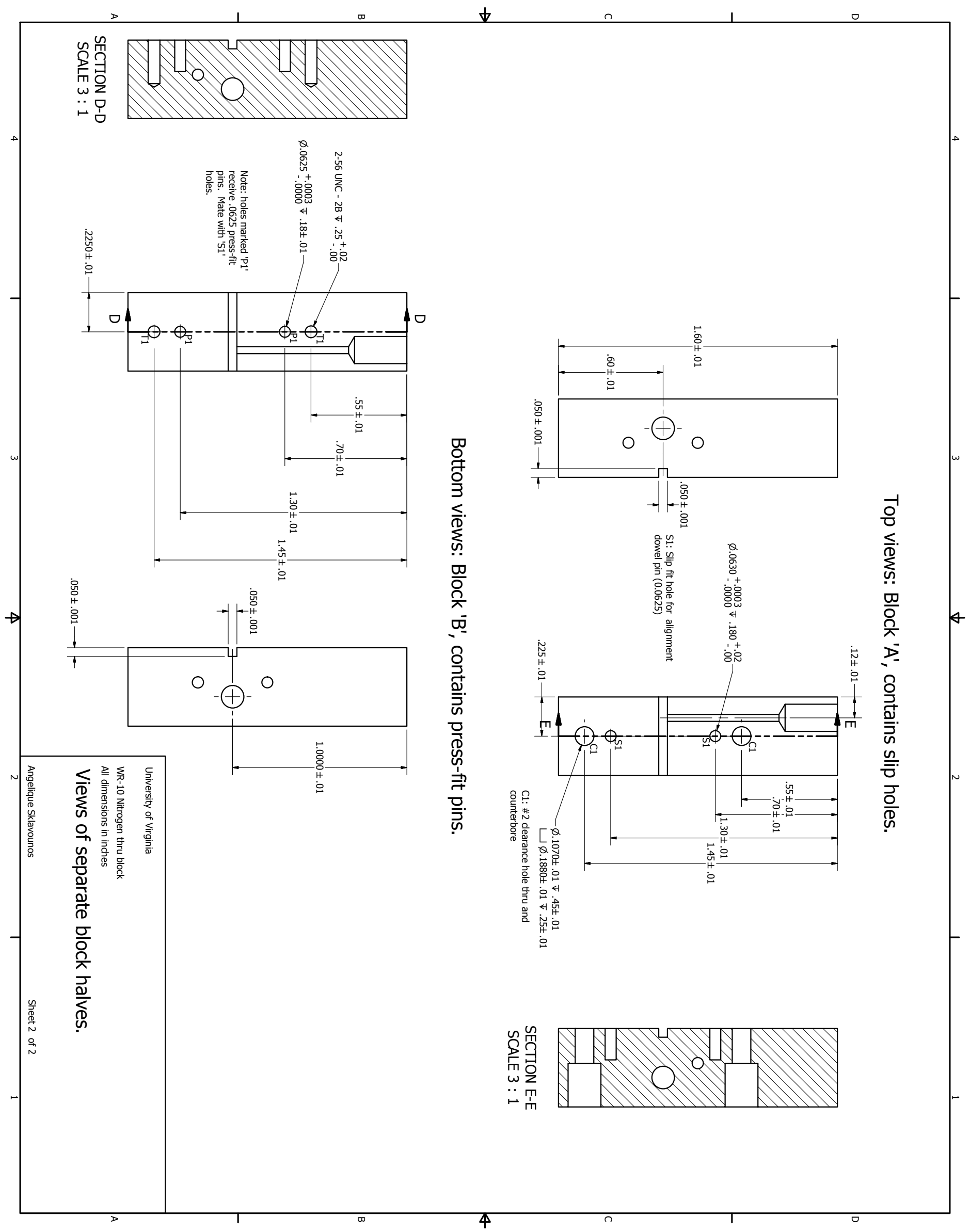




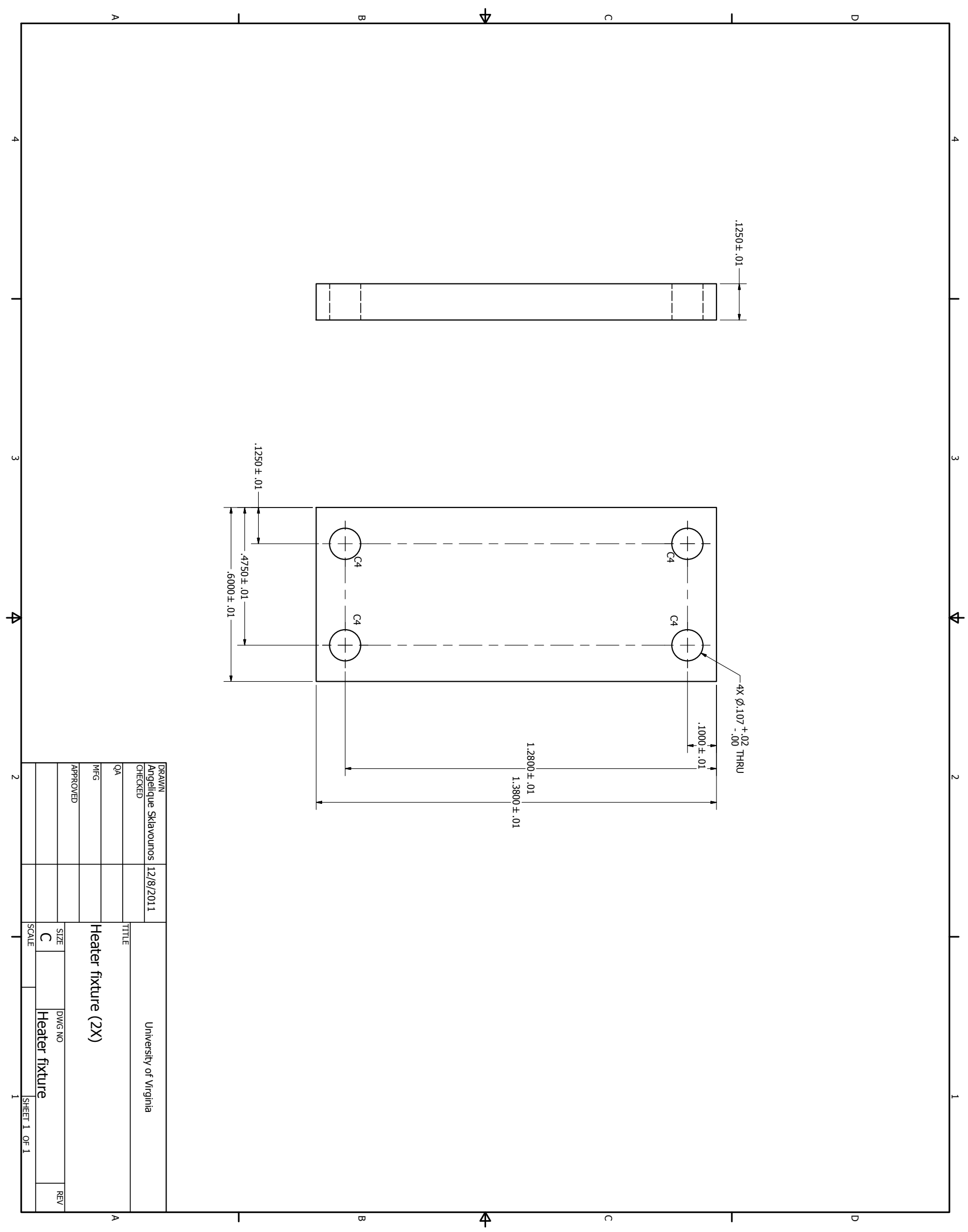




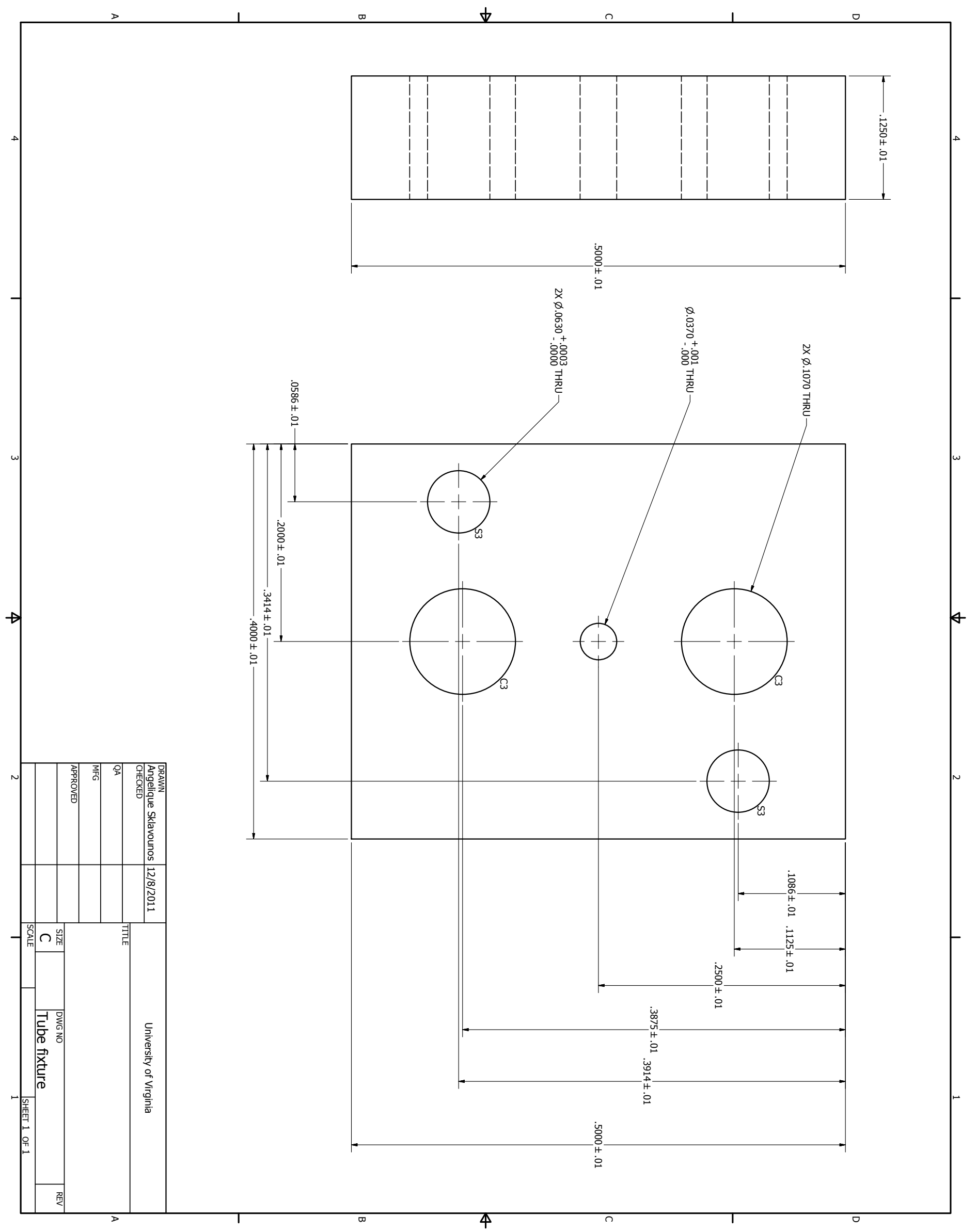




\section{Appendix C}

\section{Microwave PCR}

One use for the measurement of liquid permittivity is dielectric heating, which is found in the field of microwave chemistry [4]. Recently microwave dielectric heating has been explored for the polymerase chain reaction (PCR), which is a method for the replication of DNA. This reaction has applications in medicine and forensic sciences. It essentially consists of repeated heating and cooling cycles of the sample DNA along with a mixture of enzymes and proteins. In each cycle the mixture is heated to about $95^{\circ} \mathrm{C}$ so that denaturation of the DNA can occur, meaning the hydrogen bonds between the two strands are broken so that the DNA becomes single-stranded. Then, the sample is cooled to intermediate temperatures so that the copies of the DNA can be formed. This cycle is repeated usually thirty times on the new copies of the DNA.

Typically PCR is performed with commercial bench-top equipment and takes approximately one to two hours. Currently, there is an increasing push for for portable PCR in microchips, and the ability to combine multiple reactions onto a single chip. To accomplish this, one focus has been to localize the heating within an area on the chip, rather than heating the entire chip with the use of a hot plate in the bench-top equipment. Methods that have been demonstrated include resistive heaters, Peltier elements, and IR-mediated heating 95 98.

Likewise, with proper design, microwave heating can be localized for PCR thermal cycling. Dielectric heating occurs with the absorption of energy from the movement of rotating dipolar molecules. This effect is related to the dielectric loss of the material, so it is more effective at 
frequencies close to where the relaxation mechanism occurs. For water, which makes up the majority of PCR solutions, this relaxation occurs near $20 \mathrm{GHz}$ at room temperature. However, the loss is still considerable at frequencies between 1 and $10 \mathrm{GHz}$, for which there are cheaper and smaller microwave sources and amplifiers, thanks to the wireless industry.

The use of microwave heating for successful PCR was initially conducted with a single-mode cavity 99,100 . While this is not a localized method, as it is rather akin to a microwave oven, it showed that the components of the PCR solution were compatible with the microwave energy. A similar system involved placing a microchip inside of an X-band waveguide; PCR was successful, requiring $50 \mathrm{~W}$ of $\mathrm{RF}$ power 101 .

In order to reduce power consumption by targeting the heating to a specific micro-area, the microchip and microwave system should be designed in tandem. Recently, one design custom-fitted a machined waveguide cavity to a microchip, which reduced the RF power consumption to less than $1 \mathrm{~W}[102$. While cavities have traditionally been used for heating solutions, planar microwave circuitry is more adaptable to microchip geometry and fabrication. At the same time, however, the microchip design is guided by the electric field distribution of the planar microwave circuit. One example accomplished heating by placing a microchannel within the fields produced by coplanar waveguide, while another used a slot line-like system for the heating of water drops [103,104].

Such systems can be taken a step further by placing the liquid within strong electric fields that are induced by circuit design, such as creating standing waves along a microstrip line 105. In collaboration with Dr. James Landers, Dr. Dan Marchiarullo, Dr. Susan Barker, Kyudam Oh, and Brian Poe in the Chemistry department, the use of a microstrip matching network for efficient power transfer was investigated for low-power localized microwave heating 106 108. Within this partnership, the author of this thesis was responsible for the matching network design and measurements, along with the equipment setup for the RF heating, while the personnel in Chemistry fabricated the microchips and conducted the heating, temperature control, and PCR experiments. Computer modeling of the heating distribution within the microchip was shared equally. This appendix includes the essential RF and microchip design for efficient heating, as well as electromagnetic and thermal simulations that investigated the validity of the chamber circuit model and the temperature distribution within the chamber. The instrumentation and setup for the heating and PCR exper- 
iments is described in [106]. There was marginal success with PCR replication, but the efficiency must be increased, likely by improving the homogeneity and sensing accuracy of the temperature in the heating chamber.

\section{C.1 Microchip design}

The conceptual design of the microchip is the incorporation of the chamber and fluidic channels as part of the substrate of a microstrip circuit, within which the chamber is modeled as an electrical load, as illustrated in Figure C.1. In order to achieve low power consumption and localized heating, a microstrip matching network is designed to transform the equivalent load impedance of the heating chamber to $50 \Omega$.

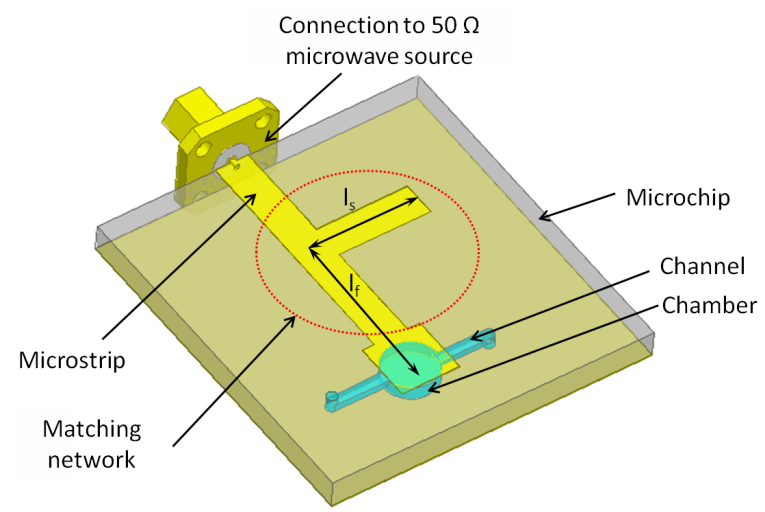

Figure C.1: Schematic of integration of microchip and microstrip network for efficient transfer of RF energy to the PCR reaction chamber.

\section{C.2 Fluidic microchip}

The fluidic microchips are fabricated from multiple polymeric layers, which each have areas cut out that form reservoir, channel, and heating well volumes when aligned and bonded together, as seen in Figure C.2. The reservoir starts at the top surface of the microchip so that the sample can be injected into the channel, which directs the sample to the heating well where the localized heating occurs. This configuration of the heating well within the microchip is very typical, and is 
well-suited for integration with microstrip, a type of microwave transmission line whose fields are located between the two conductors of the top microstrip line and the bottom ground plane.

Details of the microchip fabrication can be found in 106,109. In short, a variety of substrates were tested, starting with glass, which is easy to etch and bond but is very lossy, so the denaturation temperature could not be attained in initial experiments. Thus, a switch was made to polymeric substrates, namely polycarbonate (PC) and poly(methyl methacrylate) (PMMA). Also, due to microstrip circuit design, it is preferable to minimize the thickness of material between the top and bottom conductors in order to keep the microstrip width small and minimize the microstrip footprint. For plastics, the dielectric constant is approximately 3, thus a chip with a thickness of $2 \mathrm{~mm}$ or less would allow the microstrip width to remain below $5 \mathrm{~mm}$.

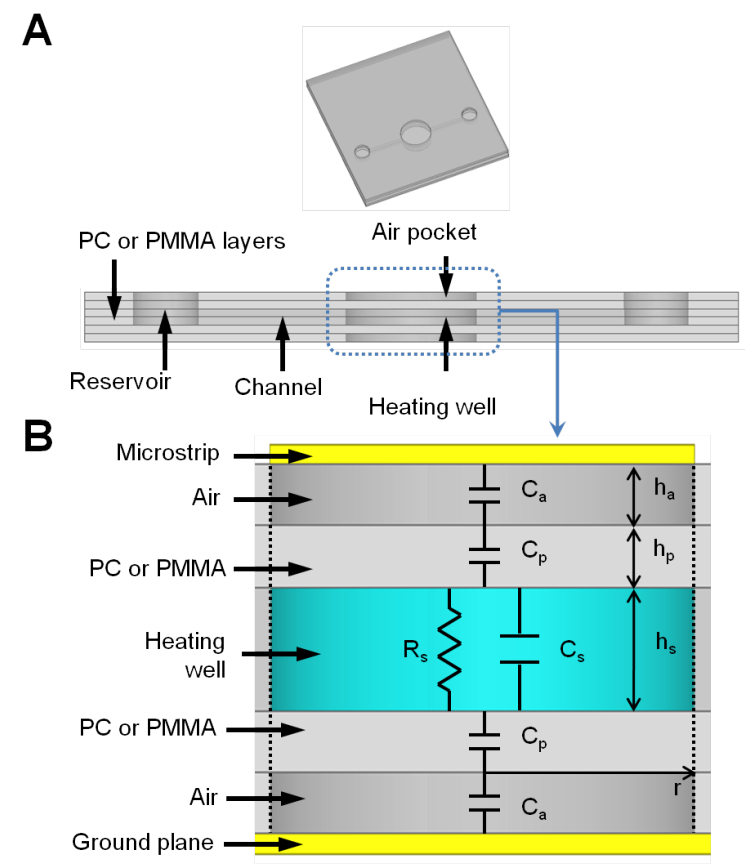

Figure C.2: A: Overall and cross-sectional views of PCR chip with air pockets and the six polymeric layers fitted together to form a chip. B: Cross-sectional view of chamber showing derivation of electrical parameters of chamber load, which consists of the solution and additional chip substrate layers.

The air pockets seen in Figure C.2 were added to reduce the non-uniformity of the chamber temperature, which was experimentally observed. Temperature distribution due to microwave heating was studied by first creating a three-dimensional electromagnetic model of the microchip and 
matching network in ANSYS HFSS, which solved for the electric fields that were next imported into ANSYS ePhysics, along with the model. Table C.1 contains thermal conductivities, densities, and specific heats for various materials (copper and constantan represented the thermocouple); room temperature was assumed to be $25{ }^{\circ} \mathrm{C}$. Simulated RF input power was adjusted to maximize the temperature close to $95{ }^{\circ} \mathrm{C}$ in order to approximate the denaturation temperatures for the PCR reaction and both static and transient heating simulations were examined. In the original chip design, as seen in Figures C.3A and B, there were both horizontal and vertical temperature gradients. The horizontal one features two hotspots at the microstrip edges, likely due to edge currents, as well as a smaller gradient in the direction perpendicular to the channel. This last effect is probably due to the asymmetrical configuration of the microstrip with regards to the chamber; the microstrip line leading to the source is bigger, so it will draw away more heat than the amount of microstrip covering the chamber in the opposite direction. The same can be said for the vertical gradient, in which the bottom of the chamber is cooler due to the larger ground plane. To mitigate this affect, air pockets were added between the chamber and the microstrip in order to reduce the heat conduction. These air pockets were also found to have helped with the horizontal gradient, but the design was further developed with guard channels, which are dummy chambers filled with solution that aid in moving the non-uniformities away from the reaction chamber. As seen in Figures C.3 C and D these added features reduced the gradient to less than $1^{\circ} \mathrm{C}$. While microchips were fabricated with the guard channels, it was difficult to completely seal the chip, so only the air pockets were included in chips for experiments.

Table C.1: Coefficients for material properties used in modeling simulations.

\begin{tabular}{|c|c|c|c|}
\hline Material & $\begin{array}{c}\text { Thermal conductivity } \\
\left(\mathrm{Wm}^{-1} \mathrm{~K}^{-1}\right)\end{array}$ & $\begin{array}{c}\text { Specific heat } \\
\left(\mathrm{Jg}^{-1} \mathrm{~K}^{-1}\right)\end{array}$ & $\begin{array}{c}\text { Mass density } \\
\left(\mathrm{gcm}^{-3}\right)\end{array}$ \\
\hline Air & 0.0257 & 1.005 & 0.0013 \\
PCR buffer (water) & 0.58 & 4.187 & 1.0 \\
Polycarbonate & 0.2 & 1.2 & 1.22 \\
Copper & 401 & 0.385 & 8.96 \\
Constantan & 19.5 & 0.39 & 8.9 \\
\hline
\end{tabular}



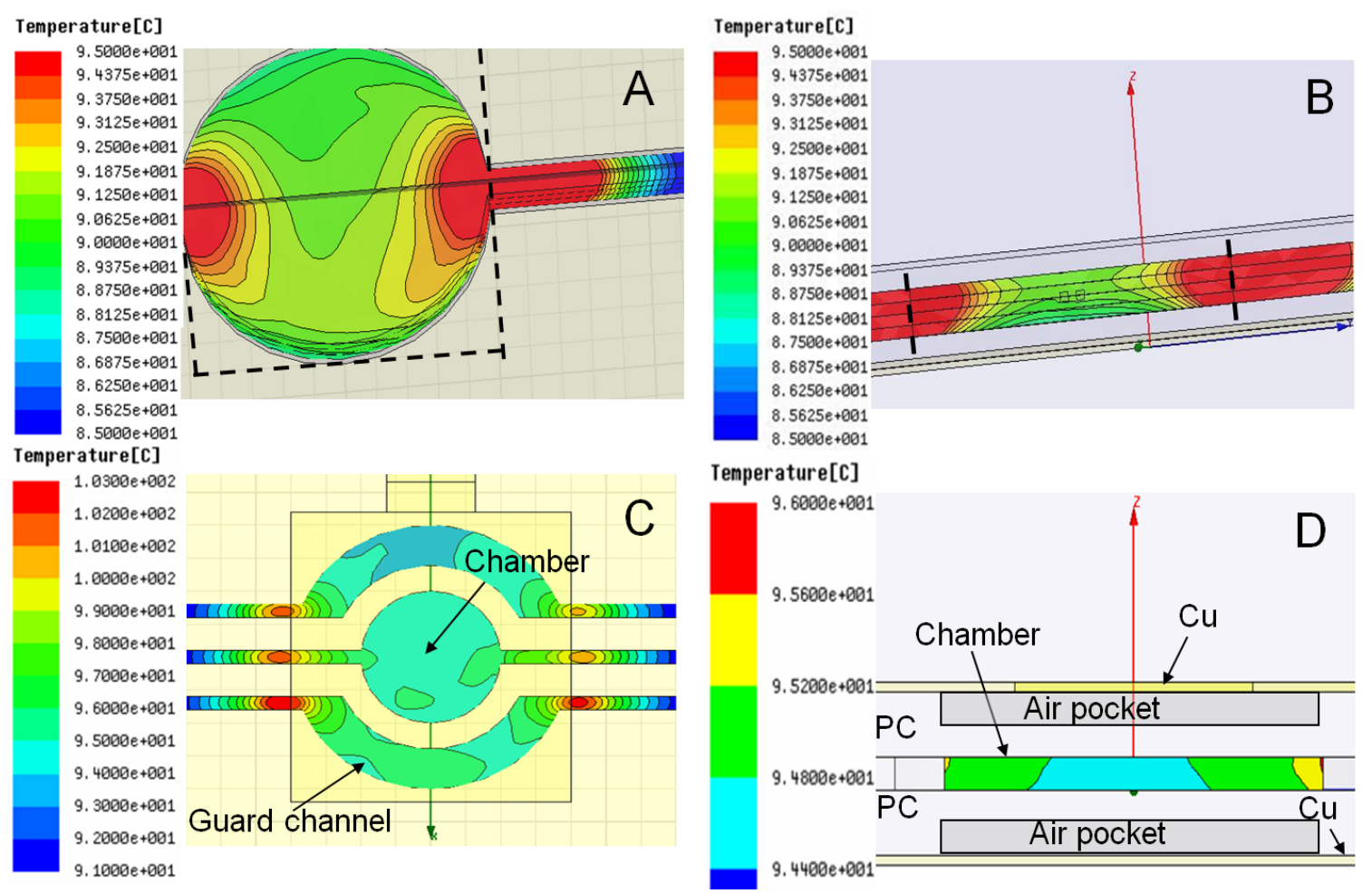

Figure C.3: Simulated temperature distributions in different chambers. A: Top view of original chamber (dashed line indicates transmission line) B: Side view of original chamber. C: Top view of chip with guard channels and air pockets. D: Side view of chip with guard channels and air pockets.

\section{C.3 Electrical design}

Microwave heating of water is based on the tendency of water molecules to rotate in order to align their permanent dipole with an applied electric field. This can be modeled as a complex permittivity:

$$
\varepsilon=\left(\varepsilon^{\prime}-j \varepsilon^{\prime \prime}\right) \varepsilon_{o}
$$

where the real component represents stored electric energy (capacitance), the imaginary component represents the energy converted to heat (resistance), and $\varepsilon_{o}$ is the vacuum permittivity. The Debye 
formula in Equation. C.1 provides a model for this permittivity as a function of frequency, $f$ :

$$
\begin{array}{r}
\varepsilon^{\prime}=\varepsilon_{\infty}+\frac{\varepsilon_{s}-\varepsilon_{\infty}}{1+(2 \pi f \tau)^{2}} \\
\varepsilon^{\prime \prime}=\frac{2 \pi f \tau\left(\varepsilon_{s}-\varepsilon_{\infty}\right)}{1+(2 \pi f \tau)^{2}}+\frac{\sigma}{2 \pi \varepsilon_{o} f}
\end{array}
$$

where $\varepsilon_{\infty}$ is the high-frequency (or optical) limit, $\varepsilon_{s}$ is the temperature-dependent static dielectric constant, $\tau$ is the temperature-dependent relaxation time constant, and $\sigma$ is the ionic conductivity [110]. Values for these parameters at room temperature are shown in Table C.2. An equivalent

Table C.2: Parameters of Debye formula $\left(\mathrm{T}=25^{\circ} \mathrm{C}\right.$, Salinity $\left.=4 \mathrm{ppt}\right)$.

\begin{tabular}{|c|c|}
\hline Parameter & \\
\hline$\varepsilon_{\infty}$ & 4.9 \\
$\varepsilon_{s}$ & 77.2 \\
$\tau$ & $8.1 \mathrm{ps}$ \\
$\sigma$ & $0.7 \Omega^{-1} \mathrm{~m}^{-1}$ \\
\hline
\end{tabular}

circuit model of the chamber, seen in Figure C.2 that represents its electrical impedance can be created using this permittivity. The equivalent resistance and capacitances of the heating chamber can be calculated as:

$$
\begin{array}{r}
R_{s}=\frac{h_{s}}{2 \pi f \varepsilon^{\prime \prime} \varepsilon_{o} \pi r^{2}}, \quad C_{s}=\frac{\varepsilon^{\prime} \varepsilon_{o} \pi r^{2}}{h_{s}} \\
C_{p}=\frac{\varepsilon_{p}^{\prime} \varepsilon_{o} \pi r^{2}}{h_{p}}, \quad C_{a}=\frac{\varepsilon_{a}^{\prime} \varepsilon_{o} \pi r^{2}}{h_{a}}
\end{array}
$$

where $h_{s}$ is the height of the heating well, $h_{p}$ is the thickness of the polymeric layers, $h_{a}$ is the thickness of the air pockets, and $r$ is the radius of the chamber; dielectric losses $\varepsilon^{\prime \prime}$ in the polymers were considered negligible. After the impedance of the chamber load was calculated, a singlestub matching circuit (Figure C.1) was designed using microstrip transmission lines. Essentially, this entails transforming the chamber impedance $Z_{L}$, which at $5.5 \mathrm{GHz}$ equals $2-j 365 \Omega$, to the microwave source impedance $Z_{0}$ of $50 \Omega$, with the parameters listed in TableC.3. First, the microstrip line of length $l_{f}$ tunes the chamber impedance to $50+j X \Omega$, then the stub of length $l_{s}$ tunes out the imaginary part of the impedance, $X$ [11]. The above model served as a guide for creating the matching network, but further tuning was still necessary and performed after chip fabrication since the simple equivalent circuit model presented above ignores second-order effects such as fringing fields between the microstrip line and the ground plane. To make the chip, the matching network 
Table C.3: $\quad$ Parameters for calculation of chamber impedance, $Z_{L}=2-j 365 \Omega$, at $f=5.8 \mathrm{GHz}$, $r=1 \mathrm{~mm}$

\begin{tabular}{|c|c|c|c|}
\hline & $\mathrm{h}(\mu \mathrm{m})$ & $\varepsilon^{\prime}$ & $\varepsilon^{\prime \prime}$ \\
\hline$R_{s}$ & 400 & - & 21.1 \\
$C_{s}$ & 400 & 72.0 & - \\
$C_{p}$ & 200 & $2.6(\mathrm{PMMA}), 2.8(\mathrm{PC})$ & - \\
$C_{a}$ & 200 & 1.0005 & - \\
\hline
\end{tabular}

was first designed using the simple model, with length $l_{s}$ of the stub made longer for tuning on the network analyzer; the circuit is cut out of copper tape and placed on the top of the chip. The ground plane consisted of a layer of copper tape affixed to a separate Plexiglas block, into which a rotatable SMA connector was screwed, allowing for multiple chip thicknesses; this setup can be seen in Figure C.4. While better power transmission is achieved if the ground plane is soldered to the SMA connector, the ground connection by mechanical contact made for faster and less wasteful experimentation. To tune the match, the chamber was filled with water or PCR buffer and the reflected power was observed on the network analyzer. The length of the stub line was trimmed until the reflected power minimum was $-15 \mathrm{~dB}$ or less and fell into the frequency range (2-7 GHz) of the microwave sources and amplifiers; an example measurement is seen in Figure C.5. The

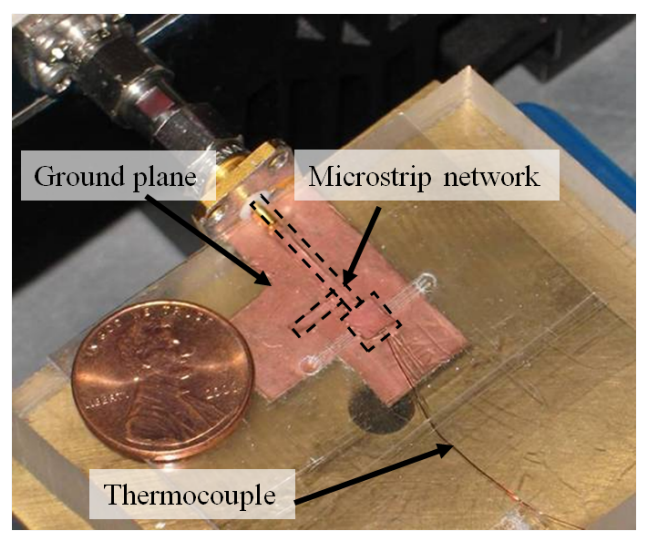

Figure C.4: Photograph of experimental PCR microchip on a copper tape-covered Plexiglas block that served as the ground plane. The black dashed line is the boundary of the matching network.

problem with the simple RC load model is that it assumes that the electric fields lie entirely within the chamber cross-section as denoted by the dashed line in Figure C.2. However, the microstrip fields do not exist wholly inside the substrate; instead, there are fringing fields on the edges of the 


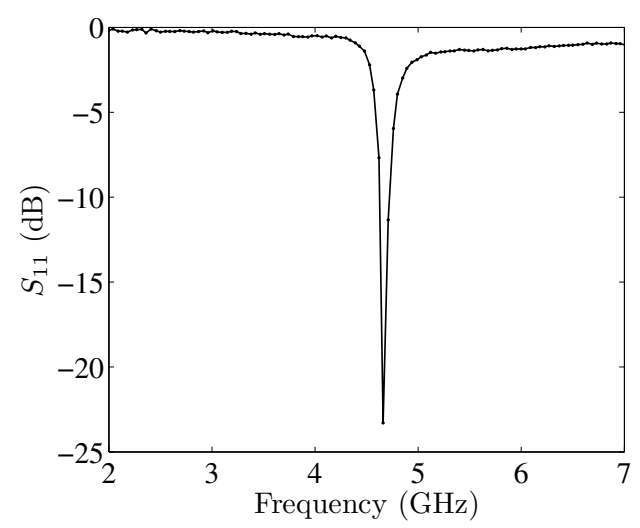

Figure C.5: Network analyzer measurement of power reflected. The dip at $4.66 \mathrm{GHz}$ indicates that the chamber is matched at this frequency.

microstrip that extend outside of the chamber cross-section. This would cause the effective radius of the additional substrate capacitances to increase, altering the chamber impedance. To test this, a model was created for a hypothetical chamber, as seen in Figure C.6, its de-embedded impedance was compared to the original RC load model in Figure C.7. As can be seen, if the radius of the chamber in the RC load model is increased, the increased capacitance reconciles the RC load model with its full-wave counterpart. More effort would be required to create a geometrically-dependent analytical model, but generally there is less fringing for shorter, wider chambers.

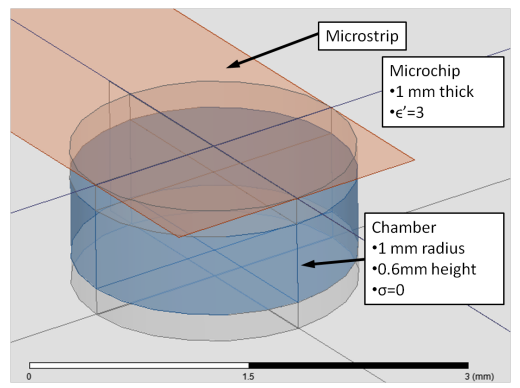

Figure C.6: HFSS setup for determination of chamber impedance. 


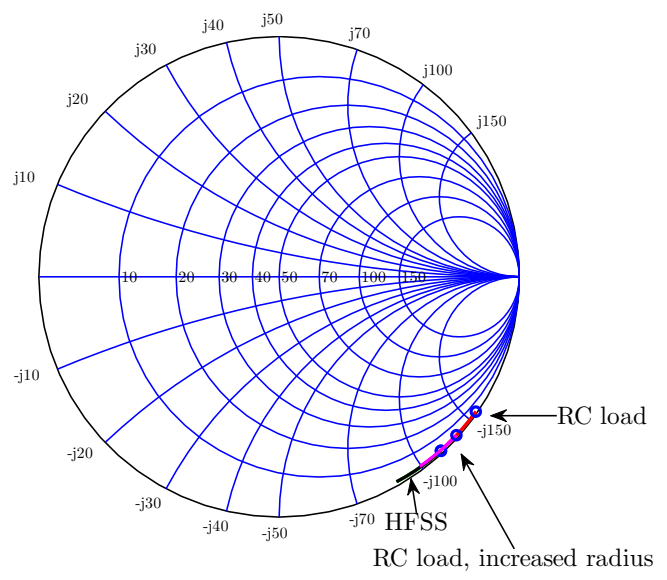

Figure C.7: Comparison of HFSS and RC load impedances. To reflect the fringing fields of microstrip, increasing the chamber radius in the RC load model shifts the impedance closer to the HFSS result. 


\section{Bibliography}

[1] J. Anderson, Dielectrics. New York: Reinhold Publishing Corporation, 1964.

[2] B. Clarke, A. Gregory, D. Cannell, M. Patrick, S. Wylie, I. Youngs, and G. Hill, "A guide to characterisation of dielectric materials at RF and microwave frequencies," Institute of Measurement and Control and the National Physical Laboratory, Tech. Rep., 2003.

[3] S. Ramo, J. R. Whinnery, and T. V. Duzer, Fields and Waves in Communication Electronics. John Wiley \& Sons, 1993.

[4] U. Kaatze and Y. Feldman, "Broadband dielectric spectrometry of liquids and biosystems," Meas. Sci. Technol., vol. 17, pp. R17-R36, February 2006.

[5] A. P. Gregory and R. N. Clarke, "A review of RF and microwave techniques for dielectric measurements on polar liquids," IEEE Transactions on Dielectrics and Electrical Insulation, vol. 13, pp. 727-743, August 2006.

[6] A. P. Gregory, R. N. Clarke, and M. G. Cox, "Traceable measurement of dielectric reference liquids over the temperature interval $10-50^{\circ} \mathrm{C}$ using coaxial-line methods," Measurement Science and Technology, vol. 20, 2009.

[7] J. T. Kindt and C. A. Schmuttenmaer, "Far-infrared dielectric properties of polar liquids probed by femtosecond terahertz pulse spectroscopy," J. Phys. Chem, vol. 100, pp. 1037310379 , March 1996.

[8] J. Barthel and R. Buchner, "High frequency permittivity and its use in the investigation of solution properties," Pure and Appl. Chem., vol. 63, 1991. 
[9] T. Globus, D. Woolard, T. W. Crowe, T. Khromova, B. Gelmont, and J. Hesler, "Terahertz fourier transform characterization of biological materials in a liquid phase," J. Phys. D: Appl. Phys., vol. 39, pp. 3405-3413, 2006.

[10] T. Globus, A. Bykhovski, T. Khromova, B. Gelmont, L. K. Tam, and L. C. Salay, "Lowterahertz spectroscopy of liquid water," in Proceedings of SPIE Vol. 6772, 2007.

[11] D. L. Woolard, E. R. Brown, M. Pepper, and M. Kemp, "Terahertz frequency sensing and imaging: a time of reckoning future applications?" Proceedings of the IEEE, vol. 93, pp. $1722-1743,2005$.

[12] R. Appleby and H. B. Wallace, "Standoff detection of weapons and contraband in the $100 \mathrm{GHz}$ to $1 \mathrm{THz}$ region," IEEE Transactions on Antennas and Propagation, vol. 55, pp. 2944-2956, 2007.

[13] N. Agmon, "Tetrahedral displacement: The molecular mechanism behind the debye relaxation in water," Journal of Physical Chemistry, vol. 100, pp. 1072-1080, 1996.

[14] W. J. Ellison, "Permittivity of pure water, at standard atmospheric pressure, over the frequency range $0-25 \mathrm{THz}$ and the temperature range $0-100^{\circ} \mathrm{C}, "$ J. Phys. Chem. Ref. Data, vol. 36, February 2007.

[15] A. Beneduci, "Which is the effective time scale of the fast Debye relaxation process in water?" Journal of Molecular Liquids, vol. 138, 2008.

[16] C. Rønne, L. Thrane, P. Åstrand, A. Wallqvist, K. Mikkelsen, and S. Keiding, "Investigation of the temperature dependence of dielectric relaxation in liquid water by $\mathrm{THz}$ reflection spectroscopy and molecular dynamics simulation," J. Chem. Phys., vol. 107, p. 5319, 1997.

[17] R. Buchner, J. Barthel, and J. Stauber, "The dielectric relaxation of water between $0^{\circ} \mathrm{C}$ and 35C," Chemical Physics Letters, vol. 306, pp. 57-63, June 1999.

[18] J. K. Vij, D. R. J. Simpson, and O. E. Panarina, "Far infrared spectroscopy of water at different temperatures: $\mathrm{GHz}$ to $\mathrm{THz}$ dielectric spectroscopy of water," Journal of Molecular Liquids, vol. 112, pp. 125-135, 2004. 
[19] S. Palese, L. Schilling, R. D. Miller, P. R. Staver, and W. T. Lotshaw, "Femtosecond optical Kerr effect studies of water," The Journal of Physical Chemistry, vol. 98, no. 25, pp. 6308-6316, 1994.

[20] E. Castner Jr, Y. Chang, Y. Chu, and G. Walrafen, "The intermolecular dynamics of liquid water," The Journal of Chemical Physics, vol. 102, p. 653, 1995.

[21] J. R. Peacock, "Millimetre wave permittivity of water near $25^{\circ} \mathrm{C}, "$ Journal of Physics D: Applied Physics, vol. 42, September 2009.

[22] M. van Exter and D. R. Grischkowsky, "Characterization of an optoelectronics terahertz beam system," IEEE Transactions on Microwave Theory and Techniques, vol. 38, pp. 1684-1691, November 1990.

[23] W. L. Chan, J. Deibel, and D. M. Mittleman, "Imaging with terahertz radiation," Reports on Progress in Physics, vol. 70, July 2008.

[24] U. Møller, D. G. Cooke, K. Tanaka, and P. U. Jepsen, "Terahertz reflection spectroscopy of debye relaxation in polar liquids [invited]," J. Opt. Soc. Am. B, vol. 26, no. 9, pp. A113-A125, 2009. [Online]. Available: http://josab.osa.org/abstract.cfm?URI=josab-26-9-A113

[25] P. U. Jepsen, U. Møller, H. Merbold et al., "Investigation of aqueous alcohol and sugar solutions with reflection terahertz time-domain spectroscopy," Opt. Express, vol. 15, no. 22, pp. 14717$14737,2007$.

[26] M. N. Afsar and K. J. Button, "Millimeter-wave dielectric measurement of materials," Proceedings of the IEEE, vol. 73, no. 1, January 1985.

[27] U. A. Khan and M. N. Afsar, "Measurement of broadband dielectric properties of cyclohexane, chlorobenzene, $10 \%$ formalin, and 1,4-dioxane using dispersive fourier transform spectroscopy," IEEE Transactions on Instrumentation and Measurement, vol. 56, no. 6, pp. 2354-2359, December 2007.

[28] M. Afsar and J. Hasted, "Measurements of the optical constants of liquid $\mathrm{H}_{2} \mathrm{O}$ and $\mathrm{D}_{2} \mathrm{O}$ between 6 and $450 \mathrm{~cm}^{-1}$," JOSA, vol. 67, no. 7, pp. 902-904, 1977. 
[29] J. Barthel, K. Bachhuber, R. Buchner, and H. Hetzenauer, "Dielectric spectra of some common solvents in the microwave region. water and lower alcohols," Chemical Physics Letters, vol. 165, no. 4, pp. 369-373, 1990.

[30] J. Barthel, K. Bachhuber, R. Buchner, H. Hetzenauer, and M. Kleebauer, "A computercontrolled system of transmission lines for the determination of the complex permittivity of lossy liquids between 8.5 and $90 \mathrm{GHz}, "$ Berichte der Bunsengesellschaft für physikalische Chemie, vol. 95, no. 8, pp. 853-859, 1991.

[31] M. Richards and R. Sheppard, "A precision waveguide system for the measurement of complex permittivity of lossy liquids and solid tissues in the frequency range $29 \mathrm{GHz}$ to $90 \mathrm{GHz}-\mathrm{II}$. the liquid system for $90 \mathrm{GHz}$; high-frequency cell design," Measurement Science and Technology, vol. 2 , no. 7 , p. $663,1991$.

[32] F. Duhamel, I. Huynen, and A. Vander Vorst, "Measurements of complex permittivity of biological and organic liquids up to $110 \mathrm{GHz}$," in Microwave Symposium Digest, 1997., IEEE MTT-S International, vol. 1. IEEE, 1997, pp. 107-110.

[33] M. Kouzai, A. Nishikata, K. Fukunaga, and S. Miyaoka, "Complex permittivity measurement at millimetre-wave frequencies during the fermentation process of Japanese sake," J. Phys. D: Appl. Phys., vol. 54-60, 2007.

[34] Z. E. Eremenko, E. M. Ganapolskii, and V. V. Vasilchenko, "Exact-calculated resonator method for permittivity measurement of high loss liquids at millimetre wavelength," Meas. Sci. Technol., vol. 16, pp. 1619-1627, July 2005.

[35] O. N. Shaforost, "Microwave whispering-gallery resonators for nanolitre liquid sensing," Ph.D. dissertation, TU Dortmund University, 2009.

[36] C. A. Balanis, Advanced enginering electromagnetics. Hoboken, NJ: John Wiley \& Sons, Inc, 1989.

[37] R. Harrington, Time-harmonic electromagnetic fields: An IEEE Press classic reissue. IEEE Press, 2001. 
[38] H. Bethe and J. Schwinger, "NDRC Report DI-117," Radiation Laboratory, MIT (March 1943), 1943.

[39] G. Birnbaum and J. Franeau, "Measurement of the dielectric constant and loss of solids and liquids by a cavity perturbation method," Journal of Applied Physics, vol. 20, no. 8, pp. 817-818, 1949 .

[40] R. Waldron, "Perturbation theory of resonant cavities," Proceedings of the IEE-Part C: Monographs, vol. 107, no. 12, pp. 272-274, 1960.

[41] U. Stumper, "A TE 01n cavity resonator method to determine the complex permittivity of low loss liquids at millimeter wavelengths," Review of Scientific Instruments, vol. 44, no. 2, pp. 165-169, 1973.

[42] S. Li, C. Akyel, and R. G. Bosisio, "Precise calculations and measurements on the complex dielectric constant of lossy materials using $\mathrm{TM}_{010}$ cavity perturbation techniques," IEEE Transactions on Microwave Theory and Techniques, vol. 29, no. 10, pp. 1041-1048, 1981.

[43] K. B. Yu, S. Ogourtsov, V. Belenky, A. Maslenikov, and A. S. Omar, "Accurate microwave resonant method for complex permittivity measurements of liquids [biological]," IEEE Transactions on Microwave Theory and Techniques, vol. 48, no. 11, pp. 2159-2164, 2000.

[44] H. Kawabata, H. Tanpo, and Y. Kobayashi, "A rigorous analysis of a TM010 mode cylindrical cavity to measure accurate complex permittivity of liquid," in Microwave Conference, 2003. 33rd European. IEEE, 2003, pp. 759-762.

[45] J. Krupka, K. Derzakowski, M. Janezic, and J. Baker-Jarvis, "TE01 $\delta$ dielectric-resonator technique for precise measurements of the complex permittivity of lossy liquids at frequencies below $1 \mathrm{GHz}$," in Precision Electromagnetic Measurements Digest, 2004 Conference on. IEEE, 2004, pp. 469-470.

[46] M. Regier and H. Schubert, "Dielectric properties at microwave frequencies studied in partially filled cylindrical TE011 cavities." The Journal of Microwave Power and Electromagnetic Energy: a publication of the International Microwave Power Institute, vol. 35, no. 1, p. 25, 2000 . 
[47] M. D. Janezic, E. F. Kuester, and J. Jarvis, "Broadband complex permittivity measurements of dielectric substrates using a split-cylinder resonator," in Microwave Symposium Digest, 2004 IEEE MTT-S International, vol. 3. IEEE, 2004, pp. 1817-1820.

[48] G. Cheng, C. Yuan, X. Ma, and L. Liu, "Multifrequency measurements of dielectric properties using a transmission-type overmoded cylindrical cavity," IEEE Transactions on Microwave Theory and Techniques, vol. 59, no. 5, pp. 1408-1418, 2011.

[49] X. Shan, Z. Shen, and T. Tsuno, "Wide-band measurement of complex permittivity using an overmoded circular cavity," Measurement Science and Technology, vol. 19, no. 2, p. 025702, 2008.

[50] R. Beyer and F. Arndt, "Efficient modal analysis of waveguide filters including the orthogonal mode coupling elements by an MM/FE method," IEEE Microwave and Guided Wave Letters, vol. 5, no. 1, pp. 9-11, 1995.

[51] X.-P. Liang, K. A. Zaki, and A. E. Atia, "A rigorous three plane mode-matching technique for characterizing waveguide T-junctions, and its application in multiplexer design," IEEE Transactions on Microwave Theory and Techniques, vol. 39, no. 12, pp. 2138-2147, 1991.

[52] T. Sieverding, U. Papziner, and F. Arndt, "Mode-matching cad of rectangular or circular multiaperture narrow-wall couplers," IEEE Transactions on Microwave Theory and Techniques, vol. 45, no. 7 , pp. 1034-1040, 1997.

[53] C. Wang, K. A. Zaki, A. E. Atia, and T. G. Dolan, "Dielectric combline resonators and filters," IEEE Transactions on Microwave Theory and Techniques, vol. 46, no. 12, pp. 2501-2506, 1998.

[54] G. V. Eleftheriades, "Analysis and desing of integrated-circuit horn antennas for millimeter and submillimeter-wave applications," Ph.D. dissertation, The University of Michigan, 1993.

[55] J. Wade and R. MacPhie, "Conservation of complex power technique for waveguide junctions with finite wall conductivity," Microwave Theory and Techniques, IEEE Transactions on, vol. 38 , no. 4 , pp. $373-378,1990$. 
[56] G. V. Eleftheriades, A. Omar, L. Katehi, and G. M. Rebeiz, "Some important properties of waveguide junction generalized scattering matrices in the context of the mode matching technique," Microwave Theory and Techniques, IEEE Transactions on, vol. 42, no. 10, pp. 1896-1903, 1994.

[57] A. Wexler, "Solution of waveguide discontinuities by modal analysis," Microwave Theory and Techniques, IEEE Transactions on, vol. 15, no. 9, pp. 508-517, 1967.

[58] Y. Rong and K. A. Zaki, "Full-wave analysis of coupling between cylindrical combline resonators," Microwave Theory and Techniques, IEEE Transactions on, vol. 47, no. 9, pp. 1721$1729,1999$.

[59] J. Zheng and M. Yu, "Rigorous mode-matching method of circular to off-center rectangular side-coupled waveguide junctions for filter applications," IEEE Transactions on Microwave Theory and Techniques, vol. 55, no. 11, pp. 2365-2373, 2007.

[60] A. Melloni, M. Politi, and G. Guido Gentili, "Mode-matching analysis of tei subi 011i/subimode waveguide bandpass filters," Microwave Theory and Techniques, IEEE Transactions on, vol. 43, no. 9, pp. 2109-2116, 1995.

[61] G. W. Hanson and A. B. Yakovlev, Operator theory for electromagnetics: an introduction. Springer Verlag, 2002.

[62] C. Yeh and G. Lindgren, "Computing the propagation characteristics of radially stratified fibers: an efficient method," Applied Optics, vol. 16, no. 2, pp. 483-493, 1977.

[63] W. H. Press, S. A. Teukolsky, W. T. Vetterling, and B. P. Flannery, Numerical recipes 2nd edition: The art of scientific computing. Cambridge university press, 2002.

[64] J. E. Marsden and M. J. Hoffman, Basic complex analysis. WH Freeman \& Company, 1999.

[65] P. Kravanja and M. Van Barel, "A derivative-free algorithm for computing zeros of analytic functions," Computing, vol. 63, no. 1, pp. 69-91, 1999.

[66] L. Delves and J. Lyness, "A numerical method for locating the zeros of an analytic function," Mathematics of computation, vol. 21, no. 100, pp. 543-560, 1967. 
[67] M.-S. Kwon and S.-Y. Shin, "Simple and fast numerical analysis of multilayer waveguide modes," Optics Communications, vol. 233, no. 1, pp. 119-126, 2004.

[68] E. Rothwell, "Computation of the logarithm of bessel functions of complex argument," Communications in numerical methods in engineering, vol. 21, no. 10, pp. 597-605, 2005.

[69] R. Mittra and S. Lee, Analytical techniques in the theory of guided waves, ser. Macmillan series in electrical science. Macmillan, 1971. [Online]. Available: http: //books.google.com/books?id=R-c8AAAAIAAJ

[70] M. D. Janezic, "Nondestructive relative permittivity and loss tangent measurements using a split-cylinder resonator," Ph.D. dissertation, University of Colorado, 2003.

[71] R. H. MacPhie and K.-L. Wu, "Scattering at the junction of a rectangular waveguide and a larger circular waveguide," IEEE Transactions on Microwave Theory and Techniques, vol. 43, no. 9, pp. 2041-2045, 1995.

[72] A. Technologies, "Agilent 85106d: Millimeter-wave network analyzer system," 1997.

[73] P. J. Petersan and S. M. Anlage, "Measurement of resonant frequency and quality factor of microwave resonators: Comparison of methods," Journal of Applied Physics, vol. 84, no. 6, pp. 3392-3402, 1998.

[74] Z. Ma, "RF properties of high temperature superconducting materials," Ph.D. dissertation, Stanford University, 1995.

[75] K. Leong and J. Mazierska, "Precise measurements of the Q factor of dielectric resonators in the transmission mode-accounting for noise, crosstalk, delay of uncalibrated lines, coupling loss, and coupling reactance," IEEE Transactions on Microwave Theory and Techniques, vol. 50, no. 9, pp. 2115-2127, 2002.

[76] P. Bevington and D. Robinson, Data Reduction and Error Analysis for the Physical Sciences. McGraw-Hill, 1992.

[77] S. Bell, "Measurement good practice guide no. 11. a beginners guide to uncertainty of measurement," Tech. rep., National Physical Laboratory, 1999. 3.2, Tech. Rep., 1999. 
[78] B. N. Taylor and C. E. Kuyatt, "Nist technical note 1297," Guidelines for evaluating and expressing the uncertainty of NIST measurement results, p. 24, 1994.

[79] J. Dutta, C. Jones, and H. Dave, "Complex dielectric constants for selected near-millimeterwave materials at 245 ghz," IEEE Transactions on Microwave Theory and Techniques, vol. 34, no. 9, pp. 932-936, 1986.

[80] M. N. Afsar and K. J. Button, "Precise millimeter-wave measurements of complex refractive index, complex dielectric permittivity and loss tangent of gaas, si, sio/sub 2/, a1/sub 2/o/sub 3/, beo, macor, and glass," Microwave Theory and Techniques, IEEE Transactions on, vol. 31, no. 2 , pp. $217-223,1983$.

[81] M. N. Afsar and H. Ding, "A novel open-resonator system for precise measurement of permittivity and loss-tangent," IEEE Transactions on Instrumentation and Measurement, vol. 50, no. 2, pp. 402-405, 2001.

[82] W. E. Courtney, "Analysis and evaluation of a method of measuring the complex permittivity and permeability microwave insulators," IEEE Transactions on Microwave Theory and Techniques, vol. 18, no. 8, pp. 476-485, 1970.

[83] M. E. Van Valkenburg, Reference Data for Engineers: Radio, Electronics, Computers and Communications. Newnes, 2001.

[84] A. N. Deleniv and S. Gevorgian, "Open resonator technique for measuring multilayered dielectric plates," Microwave Theory and Techniques, IEEE Transactions on, vol. 53, no. 9, pp. 2908-2916, 2005.

[85] Y. Zhou, E. Li, G. Guo, Y. Gao, and T. Yang, "Broadband complex permittivity measurement of low loss materials over large temperature ranges by stripline resonator cavity using segmentation calculation method," Progress In Electromagnetics Research, vol. 113, pp. 143-160, 2011.

[86] K. Lamkaouchi, A. Balana, G. Delbos, and W. Ellison, "Permittivity measurements of lossy liquids in the range 26-110 ghz," Measurement Science and Technology, vol. 14, no. 4, p. 444, 2003. 
[87] B. Jordan, R. Sheppard, and S. Szwarnowski, "The dielectric properties of formamide, ethanediol and methanol," Journal of Physics D: Applied Physics, vol. 11, no. 5, p. 695, 1978.

[88] J. Saxton, R. Bond, G. Coats, and R. Dickinson, "Dispersion at millimeter wavelengths in methyl and ethyl alcohols," The Journal of Chemical Physics, vol. 37, p. 2132, 1962.

[89] S. Szwarnowski and R. Sheppard, "Precision waveguide cells for the measurement of permittivity of lossy liquids at $70 \mathrm{GHz}, "$ Journal of Physics E: Scientific Instruments, vol. 10, no. 11, p. $1163,1977$.

[90] F. L. Penaranda-Foix, M. D. Janezic, J. M. Catala-Civera, and A. J. Canos, "Full-wave analysis of dielectric-loaded cylindrical waveguides and cavities using a new four-port ring network," Microwave Theory and Techniques, IEEE Transactions on, vol. 60, no. 9, pp. 2730-2740, 2012.

[91] C. Wang and K. A. Zaki, "Generalized multilayer anisotropic dielectric resonators," Microwave Theory and Techniques, IEEE Transactions on, vol. 48, no. 1, pp. 60-66, 2000.

[92] D. Kajfez, "Indefinite integrals useful in the analysis of cylindrical dielectric resonators," IEEE Transactions on Microwave Theory and Techniques, vol. 35, no. 9, pp. 873-874, 1987.

[93] E. Manring and J. Asmussen Jr, "Useful Bessel function identities and integrals," IEEE Transactions on Microwave Theory and Techniques, vol. 41, no. 8, pp. 1468-1471, 1993.

[94] M. M. A. El Sabbagh, "CAD of waveguide discontinuities, transitions and applications in filters and multiplexers," Ph.D. dissertation, University of Maryland, College Park, 2002.

[95] E. T. Lagally, J. R. Scherer, R. G. Blazej, N. M. Toriello, B. A. Diep, M. Ramchandani, G. F. Sensabaugh, L. W. Riley, and R. A. Mathies, "Integrated portable genetic analysis microsystem for pathogen/infectious disease detection," Analytical Chemistry, vol. 76, no. 11, pp. 3162-3170, 2004.

[96] J. Khandurina, T. E. McKnight, S. C. Jacobson, L. C. Waters, R. S. Foote, and J. M. Ramsey, "Integrated system for rapid PCR-based DNA analysis in microfluidic devices," Analytical Chemistry, vol. 72, no. 13, pp. 2995-3000, 2000. 
[97] B. C. Giordano, J. Ferrance, S. Swedberg, A. F. R. Hühmer, and J. P. Landers, "Polymerase chain reaction in polymeric microchips: DNA amplification in less than 240 seconds," Analytical Biochemistry, vol. 291, no. 1, pp. 124-132, 2001.

[98] B. C. Giordano, E. R. Copeland, and J. P. Landers, "Towards dynamic coating of glass microchip chambers for amplifying DNA via the polymerase chain reaction," Electrophoresis, vol. 22 , no. 2 , pp. $334-340,2001$.

[99] C. Fermér, P. Nilsson, and M. Larhed, "Microwave-assisted high-speed PCR," European Journal of Pharmaceutical Sciences, vol. 18, no. 2, pp. 129-132, 2003.

[100] K. Orrling, P. Nilsson, M. Gullberg, and M. Larhed, "An efficient method to perform milliliterscale PCR utilizing highly controlled microwave thermocycling," Chemical Communications, no. 7, pp. 790-791, 2004.

[101] P. Auroux, D. Reyes, J. Shah, and M. Gaitan, "Fast and efficient microfluidic PCR by microwave dielectric heating," in Proceedings Micro Total Analysis Systems, vol. 2, 2007, p. 1237.

[102] K. Shaw, P. Docker, J. Yelland, C. Dyer, J. Greenman, G. Greenway, and S. Haswell, "Rapid PCR amplification using a microfluidic device with integrated microwave heating and air impingement cooling," Lab Chip, vol. 10, no. 13, pp. 1725-1728, 2010.

[103] J. J. Shah, S. Sundaresan, J. Geist, D. Reyes, J. Booth, M. Rao, and M. Gaitan, "Microwave dielectric heating of fluids in an integrated microfluidic device," Journal of Micromechanics and Microengineering, vol. 17, p. 2224, 2007.

[104] D. Issadore, K. J. Humphry, K. A. Brown, L. Sandberg, D. A. Weitz, and R. M. Westervelt, "Microwave dielectric heating of drops in microfluidic devices," Lab on a Chip, vol. 9, pp. 1701-1706, 2009. [Online]. Available: http://dx.doi.org/10.1039/B822357B

[105] J. J. Shah, J. Geist, and M. Gaitan, "Microwave-induced adjustable nonlinear temperature gradients in microfluidic devices," Journal of Micromechanics and Microengineering, vol. 20, p. $105025,2010$. 
[106] D. Marchiarullo, A. Sklavounos, K. Oh, B. Poe, N. S. Barker, and J. Landers, "Low-power microwave-mediated heating for microchip-based PCR," Lab Chip, 2013.

[107] A. Sklavounos, D. J. Marchiarullo, S. L. R. Barker, J. P. Landers, and N. S. Barker, "Efficient miniaturized systems for microwave heating on microdevices," in Proceedings Micro Total Analysis Systems, vol. 2, 2006, pp. 1238-1240.

[108] D. J. Marchiarullo, A. Sklavounos, N. S. Barker, and J. P. Landers, "Microwave-mediated microchip thermocycling: Pathway to an inexpensive,handheld real-time pcr instrument," in Proceedings Micro Total Analysis Systems, vol. 1, 2007, p. 110.

[109] D. J. Marchiarullo, "Development of microfluidic technologies for on-site clinical and forensic analysis: Extraction, amplification, separation, and detection," Ph.D. dissertation, University of Virginia, 2010.

[110] F. Ulaby, R. Moore, and A. Fung, "Microwave remote sensing: Active and passive, vol. iii, volume scattering and emission theory, advanced systems and applications," Inc., Dedham, Massachusetts, pp. 1797-1848, 1986.

[111] D. M. Pozar, Microwave Engineering. Wiley-India, 2009. 